\title{
Catalytic Use of Elemental Gallium for Carbon-Carbon Bond Formation
}

Bo Qin and Uwe Schneider*

The University of Edinburgh, EaStCHEM School of Chemistry, The King's Buildings, David Brewster Road, Edinburgh EH9 3FJ, UK

\section{General and Additional Information}

\section{Table of Contents}

1 General Experimental $\quad$ S-2

2 Additional Experiments $\mathrm{S}-4$

2.1 Conventional Heating and Stirring: Initial Solvent Screening (Table S-1) S-4

2.2 Ultrasonic Activation: Screening of Silver Salts (Table S-2) S-5

2.3 Ultrasonic Activation: Screening of Ligands (Table S-3) S-6

$2.4 \quad$ Ultrasonic Activation: Control Experiments with $\mathrm{M}(\mathrm{OTf})_{\mathrm{x}}$ or $\mathrm{Ag}(0)$ (Table S-4) S-7

2.5 Gram-Scale Reaction at Low Catalyst Loading (Scheme S-1) S-8

2.6 Control Experiments for Asymmetric Catalysis (Table S-5) S-9

3 General Procedures for In Situ Gallium(I) Catalysis $\quad$ S-10

4 Mechanistic Experiments $S-11$

4.1 In Situ Generation of Gallium(I) Catalyst 6 from Gallium(0) S-11

4.2 Detection of Gallium(I) Catalyst 6 after C-C Bond Formation $S-11$

4.3 Stoichiometric Activation of Acetal 1a by Gallium(I) Catalyst $6 \quad S-11$

4.5 Attempted Activation of Boronic Ester 2 by Gallium(I) Catalyst $6 \quad$ S-12

4.5 Stoichiometric Activation of Boron-Ate Complex 10 by Gallium(I) Catalyst $6 \quad$ S-12

5 Analytical Data for Unknown Compounds $\quad S-14$

5.1 Analytical Data for Acetals 1a-y, Ketals 1z-z', and Aminal rac-12 S-14

5.2 Analytical Data for Homoallyl Ethers 3a-z', 3a- $\left[d_{2}\right]$, and 3'a- $\left[d_{2}\right] \quad S-16$

5.3 Analytical Data for Homopropargyl Ethers 15a and 15x S-29

5.4 Analytical Data for Silver Phosphate $(R)-17 \quad S-30$

5.5 Analytical Data for Homoallyl Amides rac-13 and $(R)-13 \quad S-31$

6 References S-32

7 Copies of NMR Spectra and Chiral HPLC Charts of Substrates and Products S-33 
Nuclear Magnetic Resonance (NMR) spectra were recorded on Bruker AVA 400, Bruker AVA 500, Bruker PRO 500, and Bruker AVA 600 spectrometers, respectively. These spectrometers operate at the following frequencies: $400 \mathrm{MHz}, 500 \mathrm{MHz}$, or $600 \mathrm{MHz}$ for ${ }^{1} \mathrm{H}$ NMR; $100 \mathrm{MHz}, 125 \mathrm{MHz}$, or $150 \mathrm{MHz}$ for ${ }^{13} \mathrm{C}$ NMR; $77 \mathrm{MHz}$ for ${ }^{2} \mathrm{H} \mathrm{NMR} ; 128 \mathrm{MHz}$ or 160 $\mathrm{MHz}$ for ${ }^{11} \mathrm{~B}$ NMR; $128 \mathrm{MHz}$ for ${ }^{19} \mathrm{~F}$ NMR; $162 \mathrm{MHz}$ or $203 \mathrm{MHz}$ for ${ }^{31} \mathrm{P}$ NMR; $152 \mathrm{MHz}$ for ${ }^{71} \mathrm{Ga} N M R$. Chemical shifts $(\delta)$ were quoted in parts per million (ppm) down-field to tetramethylsilane (TMS; $\delta=0 \mathrm{ppm}$ ), or in the scale relative to the corresponding NMR solvent used as an internal reference. Coupling constants $(\mathcal{C})$ are quoted to the nearest $0.1 \mathrm{~Hz}$. Infrared (IR) spectra were recorded on a Shimadzu IR Affinity-1 instrument using the corresponding isolated NMR sample in $\mathrm{CDCl}_{3}$ (attenuated total reflectance sampling technique). High-resolution mass spectra (HRMS) were recorded on a Finnigan MAT 900 XLT spectrometer [electrospray ionization (ESI) technique]. Chiral HPLC analyses were performed on a Shimadzu LC-20AT apparatus with an SPD-20A detector using $4.6 \times 250$ $\mathrm{mm}$ columns from DAICEL CHIRALPAK. Thin layer chromatography (TLC) was carried out on pre-coated silica gel plates from Merck (DF ALufolien $60 \mathrm{~F}_{254} ; 0.2 \mathrm{~mm}$ ). Preparative thin-layer Chromatography (PTLC) was carried out on self-prepared plates using silica gel from Wakogel (B-5F; particle size: $45 \mu \mathrm{m}$ ). Flash column chromatography was carried out using silica gel from Fisher Scientific (60 Å; particle size: 40-63 $\mu \mathrm{m}$ ). Product spots were visualized by UV light at $254 \mathrm{~nm}$ or with an appropriate stain solution.

Allyl boronic esters $\mathbf{2}^{1}$ and $2-\left[d_{2}\right]^{2}$ were prepared according to literature methods, respectively. The analytical data were in full agreement with the reported data. In this context, allyl magnesium bromide (1.0 $\mathrm{M}$ in $\mathrm{Et}_{2} \mathrm{O}$, Aldrich), trimethyl borate (99\%, Aldrich), pinacol (99\%, Alfa Aesar), vinyl boronic acid pinacol ester $(95 \%$, Aldrich), and chloroiodomethane- $\left[d_{2}\right]$ (OMX Laboratories) were used. Octyl pinacol boronic ester was also synthesized according to a literature report, ${ }^{3}$ the analytical data were in full agreement with the reported data. Allyl boronic ester MIDA complex (4; Aldrich), potassium allyl trifluoroborate (5; 95\%, Aldrich), allenyl boronic ester 14 (97\%, Aldrich), phenyl pinacol boronic ester (97\%, Aldrich), 1-(trimethylsiloxy)cyclopentene (97\%, Aldrich), and 2,6-ditert-butylpyridine (97\%, Aldrich) were purchased. Acetals 1a (99\%, Aldrich), 1h (97\%, Aldrich), 10 (97\%, TCl), 1v (98\%, Alfa Aesar), and ketal 1z' (99\%, Acros Organics) were purchased. Acetals $\mathbf{1 b}-\mathbf{g},{ }^{4} \mathbf{1 i}-\mathbf{n},{ }^{4} \mathbf{1 p},{ }^{5} \mathbf{1 q},{ }^{6} \mathbf{1 r},{ }^{7} \mathbf{1 s}-\mathbf{u},{ }^{4} \mathbf{1 y - z},{ }^{4}$ and ketal $\mathbf{1 z}$ are literatureknown and were prepared accordingly. In this context, trimethylorthoformate (99\%, Aldrich) was used. Aminal rac-12 ${ }^{8}$ is literature-known and was prepared accordingly. The analyses of these substrates were in full agreement with the reported data. Gallium metal $(99.9999 \%$, Strem) and gallium(III) triflate (98\%, Strem) were purchased; likewise, all other metal triflates for control experiments were purchased with the highest available purity. Silver triflate (99.95\%, Aldrich), silver tetrafluoroborate (99.95\%, Aldrich), silver carbonate (99.999\%, Aldrich), silver nitrate (99.995\%, Alfa Aesar), silver bistriflimide (97\%, Aldrich), silver phosphate (99\%, Alfa Aesar), silver hexafluorophosphate (99.99\%, Aldrich), silver 
hexafluoroantimonate (99\%, Alfa Aesar), silver oxide (99.99\%, Alfa Aesar), silver cyanate (99.995\%, Aldrich), silver perchlorate (anhydrous, Alfa Aesar), silver fluoride (99.9\%, Aldrich), silver chloride (99\%, Acros Organics), silver bromide (99.9\%, Alfa Aesar), silver iodide (99.999\%, Alfa Aesar), and silver metal (powder, 99.9\%, $-35+45$ mesh, Alfa Aesar) were purchased. [18]Crown-6 (99\%, Alfa Aesar), dibenzo[18]crown-6 (98\%, Alfa Aesar), [15]crown-5 (98\%, Alfa Aesar), [12]crown-4 (98\%, Aldrich), Mes-NHC (97\%, Aldrich), Dipp-NHC (97\%, Aldrich), and triphenylphosphine (99\%, Alfa Aesar) were purchased. The corresponding $(R)$-BINOL precursor to silver phosphate $(R)$-17 was purchased from Apollo Scientific Support Research Library. Unless otherwise stated, all reagents purchased from commercial suppliers were used directly without further purification. THF, toluene, and diethyl ether were distilled over sodium-benzophenone and stored over molecular sieves $(4 \AA)$ in a nitrogen glove box. All other solvents -including dioxane, benzene, mesitylene, DME, DCM, DCE, and MeCN- were used non-distilled, but stored over molecular sieves ( $4 \AA$ ) in a nitrogen glove box. Solvent dryness was confirmed using a Karl-Fischer apparatus.

All catalytic reactions were carried out in in oven-dried glassware (typically sealed $10 \mathrm{~mL}$ test tubes) under an inert atmosphere. Conventional heating and stirring was performed using a magnetic stirring bar and a hot plate magnetic stirrer (sand bath). Ultrasonic activation was carried out -in the absence of a magnetic stirring bar- using the ultrasonicator bath FB15049 from Fisherbrand (power level: 37 kHz). 


\section{Additional Experiments}

\subsection{Conventional Heating and Stirring: Initial Solvent Screening (Table S-1)}

Results for the initial screening of aromatic, etheral, and chlorinated solvents -with conventional heating and stirring- are summarized in Table S-1.

Table S-1: Initial solvent screening (conventional heating and stirring).

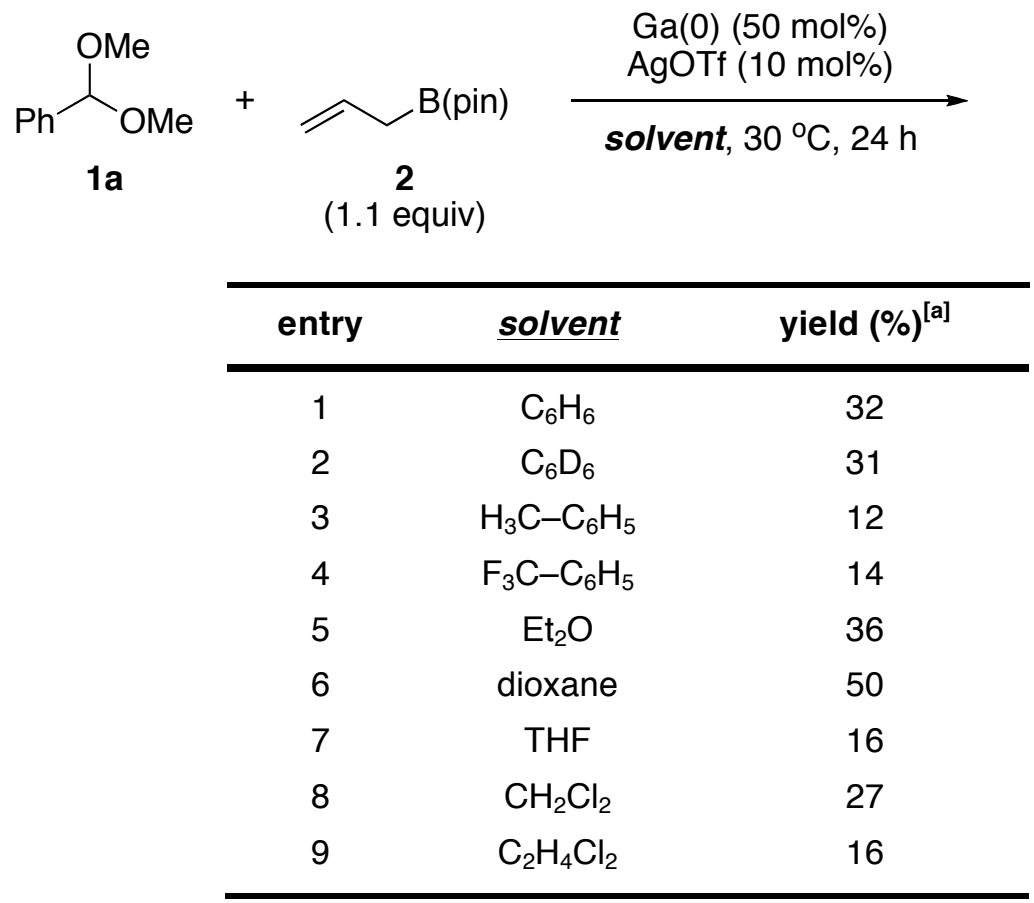

[a] The yield was determined by ${ }^{1} \mathrm{H}$ NMR spectroscopic analysis of an aliquot of the reaction mixture; internal standard: dibenzyl ether (25 mol\%).

Among all solvents examined, the use of benzene, $\mathrm{C}_{6} \mathrm{D}_{6}, \mathrm{Et}_{2} \mathrm{O}$, and dioxane proved to be most effective (TON = 3-5; entries 1, 2, 5, and 6). 


\subsection{Ultrasonic Activation: Screening of Silver Salts (Table S-2)}

Results for the screening of silver salts - under ultrasonic activation- are summarized in Table S-2.

Table S-2: Screening of silver salts (ultrasonic activation).

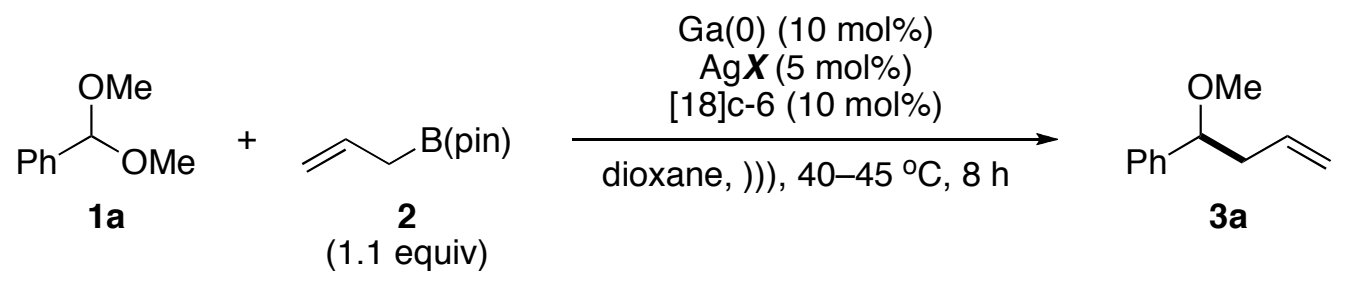

\begin{tabular}{|c|c|c|}
\hline entry & $\operatorname{Ag} \underline{X}$ & yield $(\%)^{[\mathrm{a}]}$ \\
\hline 1 & AgOTf & $99^{[b]}$ \\
\hline 2 & $\mathrm{AgBF}_{4}$ & 21 \\
\hline 3 & $\mathrm{Ag}_{2} \mathrm{CO}_{3}$ & $\mathrm{NR}^{[\mathrm{c}]}$ \\
\hline 4 & $\mathrm{AgNO}_{3}$ & $\mathrm{NR}^{[\mathrm{c}]}$ \\
\hline 5 & $\mathrm{AgNTf}_{2}$ & $\mathrm{NR}^{[\mathrm{c}]}$ \\
\hline 6 & $\mathrm{Ag}_{3} \mathrm{PO}_{4}$ & $\mathrm{NR}^{[\mathrm{c}]}$ \\
\hline 7 & $\mathrm{AgPF}_{6}$ & $\mathrm{NR}^{[\mathrm{c}]}$ \\
\hline 8 & $\mathrm{AgSbF}_{6}$ & 22 \\
\hline 9 & $\mathrm{Ag}_{2} \mathrm{O}$ & $\mathrm{NR}^{[\mathrm{c}]}$ \\
\hline 10 & $\mathrm{AgOCN}$ & 4 \\
\hline 11 & $\mathrm{AgClO}_{4}$ & 2 \\
\hline 12 & $\mathrm{AgF}$ & 63 \\
\hline 13 & $\mathrm{AgCl}$ & 60 \\
\hline 14 & $\mathrm{AgBr}$ & 5 \\
\hline 15 & $\mathrm{Agl}$ & $\mathrm{NR}^{[\mathrm{c}]}$ \\
\hline
\end{tabular}

[a] The yield was determined by ${ }^{1} \mathrm{H}$ NMR spectroscopic analysis of an aliquot of the reaction mixture; internal standard: dibenzyl ether (25 mol\%). ${ }^{[b]}$ The same result was obtained when the experiment was carried out in the presence of 2,6-di-tert-butylpyridine $(10 \mathrm{~mol} \%) .{ }_{1}{ }^{\mathrm{cc}} \mathrm{NR}=$ no reaction; the desired product $3 \mathrm{a}$ was not detected - only starting materials were recovered $\left({ }^{1} \mathrm{H}\right.$ NMR spectroscopic analysis of an aliquot of the reaction mixture).

Among all silver salts examined, AgOTf proved to be the most effective co-catalyst $(99 \%$ yield; entry 1); the participation of protons in this $\mathrm{Ga}(\mathrm{I})$ catalysis was ruled out through conducting the same experiment in the presence of a proton-trapping agent (same result). Most other salts displayed no catalytic activity, or provided $3 \mathbf{a}$ in only up to $22 \%$ yield (entries 2-11, 14, and 15). AgF and $\mathrm{AgCl}$ displayed moderate catalytic activity (60-63\% yields; entries 12 and 13). 


\subsection{Ultrasonic Activation: Screening of Ligands (Table S-3)}

Results for the screening of ligands - under ultrasonic activation- are summarized in Table S-3.

Table S-3: Screening of ligands (ultrasonic activation).

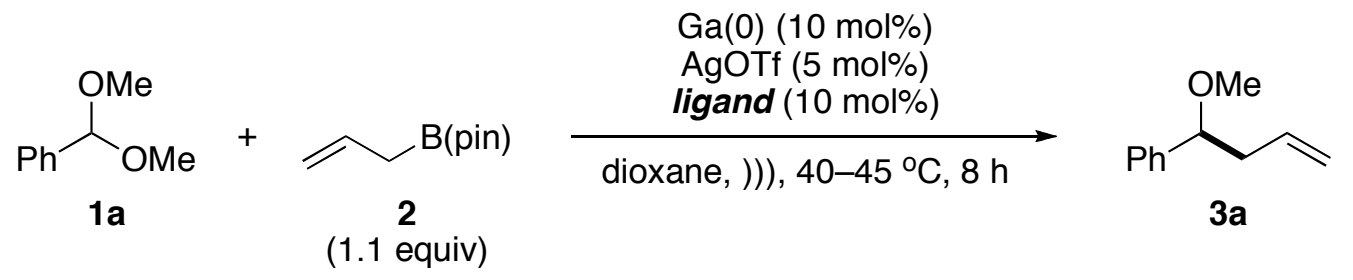

\begin{tabular}{|c|c|c|}
\hline Entry & Ligand & yield $(\%)^{[a]}$ \\
\hline 1 & - & 67 \\
\hline 2 & [18]crown-6 & 99 \\
\hline 3 & dibenzo[18]crown-6 & 67 \\
\hline 4 & [15]crown-5 & 17 \\
\hline 5 & [12]crown-4 & 73 \\
\hline 6 & & $N R^{[b]}$ \\
\hline 7 & & $N R^{[b]}$ \\
\hline 8 & $\mathrm{PPh}_{3}$ & $N R^{[b]}$ \\
\hline
\end{tabular}

[a] The yield was determined by ${ }^{1} \mathrm{H}$ NMR spectroscopic analysis of an aliquot of the reaction mixture; internal standard: dibenzyl ether $(25 \mathrm{~mol} \%)$. ${ }^{[b]} \mathrm{NR}=$ no reaction; the desired product 3a was not detected - only starting materials were recovered (' $\mathrm{H}$ NMR spectroscopic analysis of an aliquot of the reaction mixture).

Under standard conditions, in the absence of [18]crown-6, product $\mathbf{3 a}$ was obtained in 53\% yield, whereas [18]crown-6 ligation gave $3 a$ in $99 \%$ yield (entry 1 vs. entry 2). Other crown ether ligands proved to be less efficient (17-73\% yields; entries 3-5). Strong o donor ligands, such as carbenes and phosphines, shut down completely the reaction (entries 68). 


\subsection{Ultrasonic Activation: Control Experiments with $\mathrm{M}(\mathrm{OTf})_{\mathrm{x}}$ or $\mathrm{Ag}(0)$ (Table S-4)}

Results for the control experiments with other metal triflates or $\operatorname{Ag}(0)$ [being the stoichiometric by-product of the $\mathrm{Ga}(\mathrm{I})$ catalyst formation] -under ultrasonic activation- are summarized in Table S-4.

Table S-4: Control experiments with other metal triflates or $\mathrm{Ag}(0)$ (ultrasonic activation).

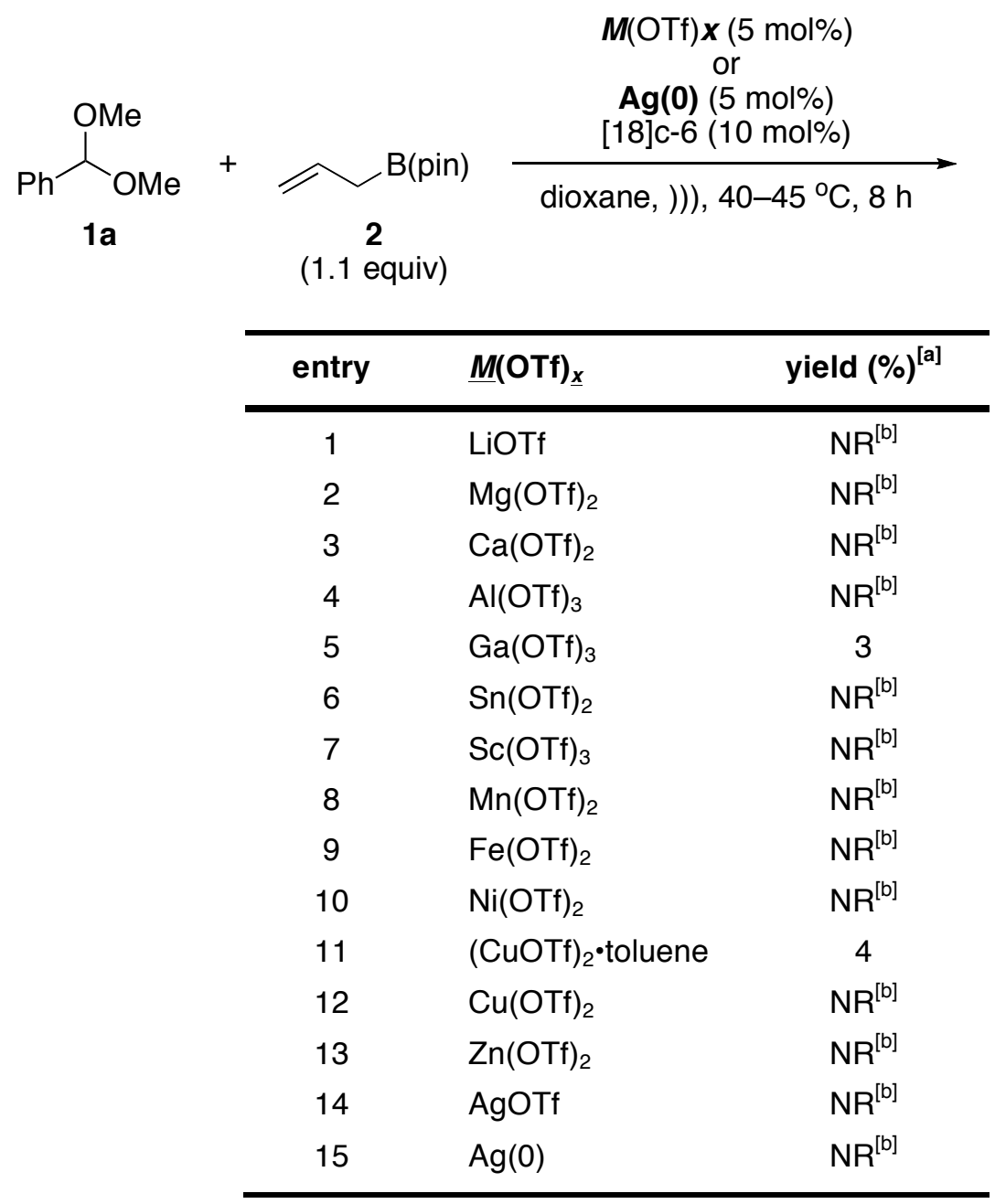

[a] The yield was determined by ${ }^{1} \mathrm{H}$ NMR spectroscopic analysis of an aliquot of the reaction mixture; internal standard: dibenzyl ether $(25 \mathrm{~mol} \%)$. ${ }^{[b]} \mathrm{NR}=$ no reaction; the desired product 3 a was not detected - only starting materials were recovered ('H NMR spectroscopic analysis of an aliquot of the reaction mixture).

Among all commercially available metal triflates examined, the vast majority led only to the recovery of starting materials; the formation of product 3a was not observed (entries 1-4, 6-10, and 12-14). In two cases, traces of product 3a were detected: $\mathrm{Ga}(\mathrm{III})$ and $\mathrm{Cu}(\mathrm{I})$ (entries 5 and 11 ). In this context, the use of $\mathrm{Ag}(0)$ also failed to give $\mathbf{3 a}$ (entry 15). 


\subsection{Gram-Scale Reaction at Low Catalyst Loading (Scheme S-1)}

The result for an appropriate gram-scale experiment at low catalyst loading is summarized in Scheme S-1.

Scheme S-1: Gram-scale experiment at low catalyst loading.

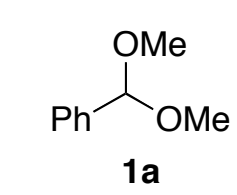

$(1.52 \mathrm{~g}, 10.0 \mathrm{mmol})$

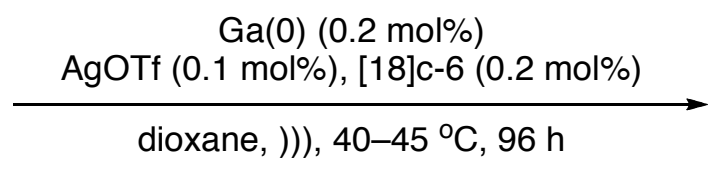

$\gamma^{\mathrm{B}(\text { pin })}$

2

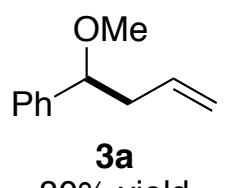

$80 \%$ yield

\section{(1.1 equiv)}

The use of $0.2 \mathrm{~mol} \%$ of elemental gallium -combined with $0.1 \mathrm{~mol} \%$ of silver(l) triflate and $0.2 \mathrm{~mol} \%$ of [18]crown-6- is expected to give $0.1 \mathrm{~mol} \%$ of the in situ gallium(I) catalyst = actual catalyst loading. 


\subsection{Control Experiments for Asymmetric Catalysis (Table S-5)}

Results regarding control experiments for asymmetric catalysis -with conventional heating and stirring- are summarized in Table S-5.

Table S-5: Control experiments for asymmetric catalysis (conventional heating and stirring).

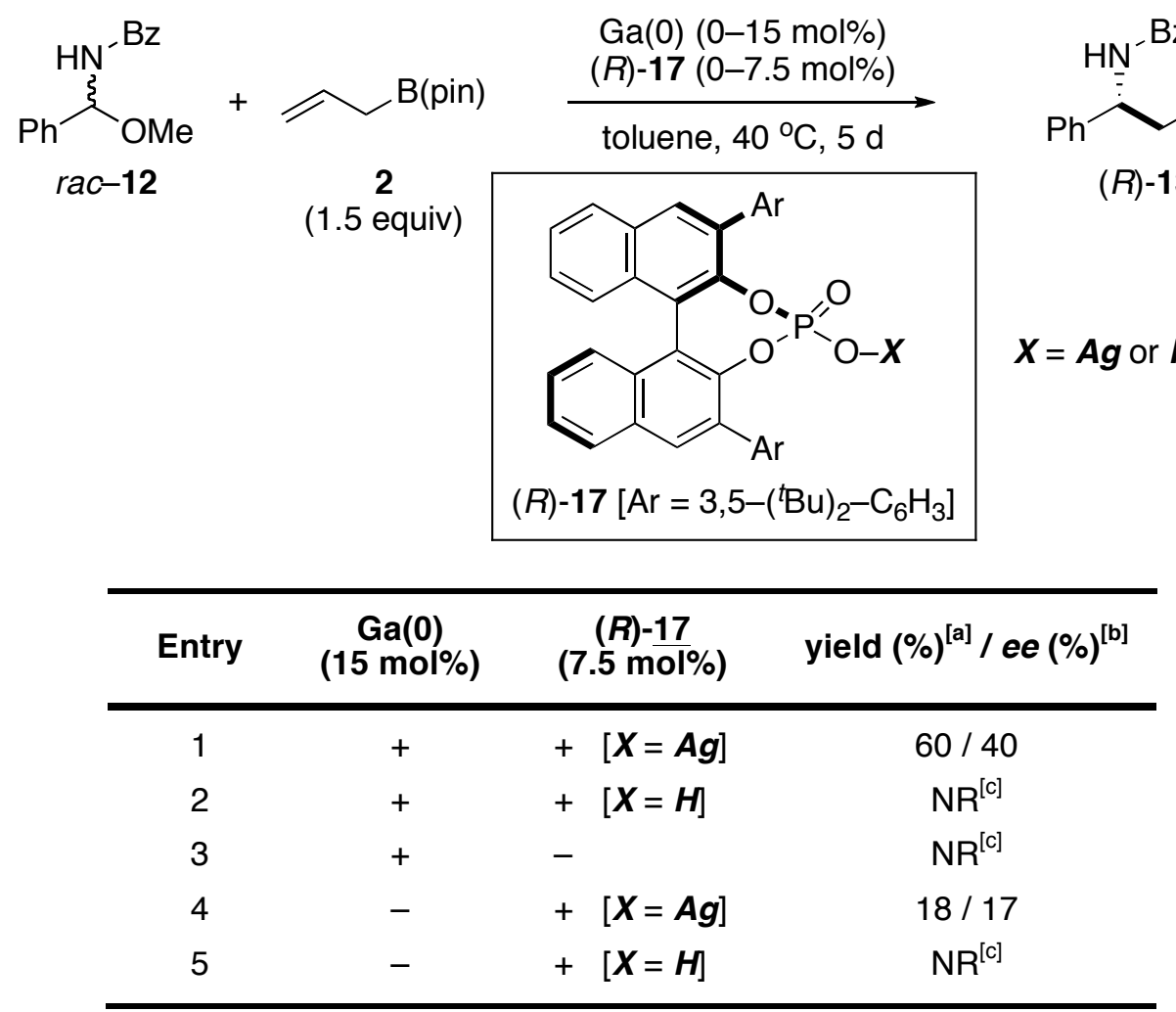

[a] The yield was determined by ${ }^{1} \mathrm{H}$ NMR spectroscopic analysis of an aliquot of the reaction mixture; internal standard: dibenzyl ether $(25 \mathrm{~mol} \%)$. [b] The enantiomeric excess $(e e)$ was determined by chiral HPLC analysis of a purified sample of $13 .{ }^{[c]} \mathrm{NR}=$ no reaction; the desired product 13 was not detected - only starting materials were recovered $\left({ }^{1} \mathrm{H}\right.$ NMR spectroscopic analysis of an aliquot of the reaction mixture).

The combined use of gallium(0) and silver salt $(R)-\mathbf{1 7}(X=A g)$ gave product $(R)-\mathbf{1 3}$ in $60 \%$ yield with $40 \%$ ee (entry 1$)$. The combination of gallium(0) and acid $(R)-17(X=H)$ or the sole use of gallium( 0 ) failed to catalyze this reaction (entries 2 and 3 ). In the absence of gallium(0), the sole use of silver salt $(R)-\mathbf{1 7}(X=A g)$ or acid $(R)-\mathbf{1 7}(X=H)$ gave poor results (entries 4 and 5 ). 


\section{General Procedure A [Standard Conditions = Ultrasonic Activation]}

Gallium(0) (1.4 mg, $0.02 \mathrm{mmol}, 10 \mathrm{~mol} \%)$, silver triflate $(2.8 \mathrm{mg}, 0.01 \mathrm{mmol}, 5 \mathrm{~mol} \%)$ or silver fluoride (1.3 mg, $0.01 \mathrm{mmol}, 5 \mathrm{~mol} \%$ ), [18]crown-6 (5.2 mg, $0.02 \mathrm{mmol}, 10 \mathrm{~mol} \%$ ), the corresponding acetal/ketal $1(0.20 \mathrm{mmol})$, allyl boronic ester 2 (37.0-50.4 $\mathrm{mg}, 0.22-$ $0.30 \mathrm{mmol}, 1.1-1.5$ equiv), and dioxane $(200 \mu \mathrm{L}, 1.0 \mathrm{M})$ or toluene $(400-800 \mu \mathrm{L}, 0.25-0.50$ $\mathrm{M})$ were added to an oven-dried $10 \mathrm{~mL}$ test tube in a nitrogen-filled glove box. The mixture was reacted under ultrasonic activation at $40-50{ }^{\circ} \mathrm{C}$ for $8-78 \mathrm{~h}$ (inert atmosphere), at which point ${ }^{1} \mathrm{H}$ NMR spectroscopic analysis indicated the complete consumption of 1 . The solvent was removed in vacuo, and the residue was purified by PTLC on silica gel -as indicated- to give the corresponding homoallyl ether 3 .

Alternatively, allyl boronic ester 2-[ $\left.d_{2}\right](51.0 \mathrm{mg}, 0.30 \mathrm{mmol}, 1.5$ equiv) and allenyl boronic ester 14 (39.9 mg, 0.24 mmol, 1.2 equiv) have been used instead of 2 under otherwise identical conditions. Likewise, aminal rac-12 (48.3 $\mathrm{mg}, 0.20 \mathrm{mmol})$ has been used instead of 1 ; in this specific case ultrasonic activation was not required.

\section{General Procedure B [Gram-Scale \& Low Catalyst Loading]}

Gallium(0) (1.4 mg, $0.02 \mathrm{mmol}, 0.20 \mathrm{~mol} \%)$, silver triflate (2.8 mg, $0.01 \mathrm{mmol}, 0.10 \mathrm{~mol} \%)$, [18]crown-6 (5.2 mg, $0.02 \mathrm{mmol}, 10 \mathrm{~mol} \%)$, acetal 1a (1.52 g, $10.0 \mathrm{mmol})$, allyl boronic ester 2 ( $1.85 \mathrm{~g}, 11.0 \mathrm{mmol}, 1.1$ equiv), and dioxane (10 mL, $1.0 \mathrm{M})$ were added to an ovendried $50 \mathrm{~mL}$ round-bottom flask in a nitrogen-filled glove box. The mixture was reacted under ultrasonic activation at $40-45{ }^{\circ} \mathrm{C}$ for $96 \mathrm{~h}$ (inert atmosphere). The solvent was removed in vacuo, and the residue was purified by flash column chromatography on silica gel (eluent: heptane/EtOAc $=19: 1)$ to give homoallyl ether $3 \mathrm{a}$ in $80 \%$ yield $(1.30 \mathrm{~g})$ as a pale yellow liquid.

\section{General Procedure C [Asymmetric Version]}

Gallium(0) (2.1 mg, $0.03 \mathrm{mmol}, 15 \mathrm{~mol} \%)$, silver phosphate $(R)-17(8.3 \mathrm{mg}, 0.015 \mathrm{mmol}$, $7.5 \mathrm{~mol} \%$ ), aminal rac-12 (48.3 mg, $0.20 \mathrm{mmol})$, allyl boronic ester 2 (50.4 mg, $0.30 \mathrm{mmol}$, 1.5 equiv), and toluene (200 $\mu \mathrm{L}, 1.0 \mathrm{M})$ were added to an oven-dried $10 \mathrm{~mL}$ test tube with a magnetic stirring bar in a nitrogen-filled glove box. The reaction mixture was stirred at 40 ${ }^{\circ} \mathrm{C}$ for 5 days (inert atmosphere). The solvent was removed in vacuo, and the residue was purified by PTLC on silica gel [eluent: $\mathrm{PE}(40-60) / \mathrm{Et}_{2} \mathrm{O}=5: 1$, eluted 4 times] to give homoallyl amide 13 -with predominant $R$ configuration- as a colorless solid. The optical purity of the product was determined by chiral HPLC analysis (DAICEL CHIRALPAK IF). 


\subsection{In Situ Generation of Gallium(I) Catalyst $\underline{6}$ from Gallium(0)}

Gallium metal (27.9 mg, $0.40 \mathrm{mmol})$, silver triflate $(51.4 \mathrm{mg}, 0.20 \mathrm{mmol}, 0.5$ equiv), [18]crown-6 (106 mg, $0.40 \mathrm{mmol}, 1$ equiv), and dioxane (400 $\mu \mathrm{L}, 1.0 \mathrm{M})$ were added to an oven-dried $10 \mathrm{~mL}$ test tube in a nitrogen-filled glove box. The mixture was reacted under ultrasonic activation at $40-45{ }^{\circ} \mathrm{C}$ for $36 \mathrm{~h}$ (inert atmosphere), at which point the reaction mixture (100 $\mu \mathrm{L}$ aliquot) was analyzed by ${ }^{71} \mathrm{Ga}$ NMR spectroscopy (Young NMR tube). The spectrum displayed a single resonance at $-566 \mathrm{ppm}$, which has been ascribed -in analogy to literature- to a novel gallium(I) species, i.e., gallium(I) catalyst 6: [18]c-6$\mathrm{Ga}(\mathrm{I}) \cdot(\text { dioxane })_{n} \mathrm{OTf}(n=1,2,3)$. Efforts to isolate or further characterize this highly reactive species by ${ }^{1} \mathrm{H} N M R$ and ${ }^{13} \mathrm{C} N M R$ spectroscopy, HRMS, or $X$-ray analysis failed to give conclusive results. Evidence for $\mathrm{Ga}(\mathrm{II})$ or $\mathrm{Ga}(\mathrm{III})$ species were not detected under these conditions.

${ }^{71} \mathrm{Ga} \operatorname{NMR}\left(\mathrm{C}_{6} \mathrm{D}_{6}, 152 \mathrm{MHz}\right): \delta=-566 \mathrm{ppm}(\mathbf{6})$.

\subsection{Detection of Gallium(I) Catalyst $\underline{6}$ after C-C Bond Formation}

Acetal 1a (30.4 mg, $0.20 \mathrm{mmol}, 1$ equiv) and allyl boronic ester 2 (37.0 mg, $0.22 \mathrm{mmol}, 1.1$ equiv) were added to the pre-formed solution of gallium(I) catalyst $6(0.20 \mathrm{mmol}$; see 4.1$)$ in a nitrogen-filled glove box and kept at $40{ }^{\circ} \mathrm{C}$ for $4 \mathrm{~h}$ (Young NMR tube), at which point the reaction mixture was analyzed by ${ }^{11} \mathrm{~B}$ and ${ }^{71} \mathrm{Ga}$ NMR spectroscopy (100 $\mu \mathrm{L}$ aliquot). The ${ }^{11} \mathrm{~B}$ NMR spectrum displayed the appearance of a novel major resonance at $22 \mathrm{ppm}$, which has been ascribed to the stoichiometric by-product of the $\mathrm{C}-\mathrm{C}$ bond formation between 1a and 2, MeO-B(pin) (7); a second minor signal at 33 ppm, corresponding to residual 2, was detected as well. In this context, ${ }^{1} \mathrm{H} N M R$ spectroscopy confirmed the formation of homoallyl ether $3 a$ in $>99 \%$ yield. The ${ }^{71} \mathrm{Ga}$ NMR spectrum displayed a single resonance at $-587 \mathrm{ppm}$, which has been ascribed to the regenerated gallium(I) catalyst 6 (which is now in a slightly different medium, hence, a slightly different chemical shift was observed). Evidence for $\mathrm{Ga}(\mathrm{II})$ or $\mathrm{Ga}$ (III) species were not detected under these conditions.

${ }^{11} \mathrm{~B}$ NMR $\left(\mathrm{C}_{6} \mathrm{D}_{6}, 128 \mathrm{MHz}\right): \delta=33 \mathrm{ppm}$ (minor = residual 2), $22 \mathrm{ppm}$ ( major $=7$ ).

${ }^{71} \mathrm{Ga}$ NMR $\left(\mathrm{C}_{6} \mathrm{D}_{6}, 152 \mathrm{MHz}\right): \delta=-587 \mathrm{ppm}$ (regenerated 6).

\subsection{Stoichiometric Activation of Acetal 1 a by Gallium(I) Catalyst $\underline{6}$}

Acetal 1a (30.4 mg, $0.20 \mathrm{mmol}, 1$ equiv) was added to the pre-formed solution of gallium(I) catalyst $6(0.20 \mathrm{mmol}$; see 4.1$)$ in a nitrogen-filled glove box and kept at $40{ }^{\circ} \mathrm{C}$ for $2 \mathrm{~h}$ (Young NMR tube), at which point the reaction mixture was analyzed by HRMS (ESI) and ${ }^{71} \mathrm{Ga}$ NMR spectroscopy (100 $\mu \mathrm{L}$ aliquot). The analysis by HRMS (ESI) revealed the formation of the corresponding oxocarbenium ion species 8. [In this context, 8 was not detected when a mixture of 1a, AgOTf, and [18]c-6 was used under otherwise identical 
conditions (no gallium)]. Accordingly, the concomitant formation of a gallium(I) methoxide species 9, [Ga(I)]-OMe, may be concluded. The ${ }^{71} \mathrm{Ga}$ NMR spectrum did not display any signal, which is in accordance with literature precedents of electron-rich gallium(I) complexes bearing phosphine or carbene ligands. Efforts to isolate or further characterize this highly reactive species by ${ }^{1} \mathrm{H} N M R$ and ${ }^{13} \mathrm{C} N M R$ spectroscopy, HRMS, or X-ray analysis failed to give conclusive results. Evidence for $\mathrm{Ga}$ (II) or $\mathrm{Ga}$ (III) species were not detected under these conditions. After addition of 2 to the mixture of 8 and 9, homoallyl ether 3 a was smoothly formed in $>99 \%$ yield ( ${ }^{1} H$ NMR spectroscopy).

HRMS (ESI): calculated for $\mathrm{C}_{8} \mathrm{H}_{9} \mathrm{O}^{+}=[\mathrm{M}]^{+}: \mathrm{m} / \mathrm{z}=121.0679$, found: $\mathrm{m} / \mathrm{z}=121.0671$ (8).

${ }^{71} \mathrm{Ga}$ NMR $\left(\mathrm{C}_{6} \mathrm{D}_{6}, 152 \mathrm{MHz}\right)$ : no signal (9).

\subsection{Attempted Activation of Boronic Ester $\underline{2}$ by Gallium(I) Catalyst 6}

Allyl boronic ester 2 ( $37.0 \mathrm{mg}, 0.22 \mathrm{mmol}, 1.1$ equiv) was added to the pre-formed solution of gallium(I) catalyst $6(0.20 \mathrm{mmol}$; see 4.1$)$ in a nitrogen-filled glove box and kept at $40{ }^{\circ} \mathrm{C}$ for $24 \mathrm{~h}$ (Young NMR tube), at which point the reaction mixture was analyzed by ${ }^{11} \mathrm{~B}$ and ${ }^{71} \mathrm{Ga}$ NMR spectroscopy (100 $\mu \mathrm{L}$ aliquot). The ${ }^{11} \mathrm{~B}$ NMR spectrum displayed a single resonance at $33 \mathrm{ppm}$, which corresponds to remaining 2. The ${ }^{71} \mathrm{Ga}$ NMR spectrum displayed a single resonance at $-566 \mathrm{ppm}$, which corresponds to remaining 6 . Thus, a reaction did not occur under these conditions; evidence for $\mathrm{Ga}$ (II) or $\mathrm{Ga}$ (III) species were not detected neither.

${ }^{11} \mathrm{~B}$ NMR $\left(\mathrm{C}_{6} \mathrm{D}_{6}, 128 \mathrm{MHz}\right): \delta=33 \mathrm{ppm}(2 ;$ no change).

${ }^{71} \mathrm{Ga} \operatorname{NMR}\left(\mathrm{C}_{6} \mathrm{D}_{6}, 152 \mathrm{MHz}\right): \delta=-566 \mathrm{ppm}(6 ;$ no change) .

\subsection{Stoichiometric Activation of Boron-Ate Complex 10 by Gallium(I) Catalyst $\underline{6}$}

Allyl boronic ester 2 (37.0 mg, $0.22 \mathrm{mmol}, 1.1$ equiv) and K-OMe (14.0 mg, $0.20 \mathrm{mmol})$ were reacted in dioxane $(200 \mu \mathrm{L}, 1.0 \mathrm{M})$ at $40{ }^{\circ} \mathrm{C}$ for $18 \mathrm{~h}$ to form the corresponding boron-ate complex 10 , allyl- $\mathrm{B}^{-}(\mathrm{OMe})(\mathrm{pin}) \mathrm{K}^{+}$. The generation of this species was confirmed by ${ }^{11} \mathrm{~B}$ NMR spectroscopic analysis, which displayed two signals at $33 \mathrm{ppm}$ (minor; residual 2) and $7 \mathrm{ppm}$ (major; 10). The solution of $\mathbf{1 0}$ was added to the pre-formed solution of gallium(I) catalyst $6(0.20 \mathrm{mmol}$; see 4.1$)$ in a nitrogen-filled glove box and kept at $40{ }^{\circ} \mathrm{C}$ for $2 \mathrm{~h}$ (Young NMR tube), at which point the reaction mixture was analyzed by ${ }^{11} \mathrm{~B}$ and ${ }^{71} \mathrm{Ga} N \mathrm{NM}$ spectroscopy (100 $\mu \mathrm{L}$ aliquot). The ${ }^{11} \mathrm{~B}$ NMR spectrum displayed the appearance of a novel major resonance at $22 \mathrm{ppm}$, which has been ascribed to the stoichiometric by-product of the B-Ga transmetalation between 10 and 6, MeO-B(pin) (7); a second minor signal at $33 \mathrm{ppm}$, corresponding to residual 2, was detected alongside with a trace of $10(7 \mathrm{ppm})$. The ${ }^{71} \mathrm{Ga}$ NMR spectrum revealed a novel single resonance at -624 ppm, which has been ascribed to unprecedented allyl gallium(I) species 11 (likely $\sigma$ and $\pi$ complexes in equilibrium). Efforts to isolate or further characterize this highly reactive species by ${ }^{1} \mathrm{H}$ NMR and ${ }^{13} \mathrm{C}$ NMR spectroscopy, HRMS, or $X$-ray analysis failed to give 
conclusive results. Evidence for $\mathrm{Ga}(\mathrm{II})$ or $\mathrm{Ga}$ (III) species were not detected under these conditions.

${ }^{11} \mathrm{~B}$ NMR $\left(\mathrm{C}_{6} \mathrm{D}_{6}, 128 \mathrm{MHz}\right): \delta=33 \mathrm{ppm}$ (minor = residual 2), $22 \mathrm{ppm}$ (major $=7$ ), $7 \mathrm{ppm}$ (trace $=10$ ).

${ }^{71} \mathrm{Ga}$ NMR $\left(\mathrm{C}_{6} \mathrm{D}_{6}, 152 \mathrm{MHz}\right): \delta=-624 \mathrm{ppm}(11)$. 


\section{$5 \quad$ Analytical Data for Unknown Compounds}

\subsection{Analytical Data for Acetals 1a-y, Ketals 1z-z', and Aminal rac-12}

The synthesized acetals $\mathbf{1 b},{ }^{9} \mathbf{1 c},{ }^{10} \mathbf{1 d},{ }^{11} \mathbf{1 e}-\mathbf{g},{ }^{12} \mathbf{1 i},{ }^{13} \mathbf{1 j}-\mathbf{n},{ }^{12} \mathbf{1 p},{ }^{5} \mathbf{1 q},{ }^{6} \mathbf{1 r},{ }^{7} \mathbf{1 s - t},{ }^{14} \mathbf{1 w}-\mathbf{y},{ }^{12}$ ketal $1 z,{ }^{15}$ and aminal rac- $12^{8}$ are literature-known, and thee obtained analytical data are in full agreement with the reported data. The obtained analytical data for the novel acetals $\mathbf{1} \mathbf{u}-\mathbf{v}$ and ketal 1z' are listed below.

\section{2-(Dimethoxymethyl)benzo[b]thiophene (1u)}

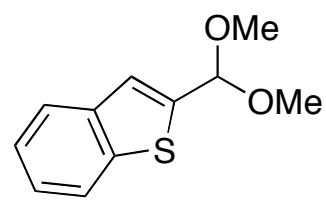

$1 \mathbf{u}$

Pale yellow liquid.

${ }^{1} \mathrm{H}$ NMR $\left(\mathrm{CDCl}_{3}, 600 \mathrm{MHz}\right): \delta=3.41(\mathrm{~s}, 6 \mathrm{H}), 5.71(\mathrm{~s}, 1 \mathrm{H}), 7.29-7.37(\mathrm{~m}, 3 \mathrm{H}), 7.75(\mathrm{~d}, J=$ $7.2 \mathrm{~Hz}, 1 \mathrm{H}), 7.82(\mathrm{~d}, J=7.2 \mathrm{~Hz}, 1 \mathrm{H}) \mathrm{ppm}$.

${ }^{13} \mathrm{C}$ NMR $\left(\mathrm{CDCl}_{3}, 150 \mathrm{MHz}\right): \delta=52.7(2 \mathrm{C}), 100.2,122.3,122.5,123.8,124.3,124.4,139.4$, 140.0, $142.3 \mathrm{ppm}$.

IR (neat): $v=2933,1458,1435,1346,1186,1139,1042,977,744,725 \mathrm{~cm}^{-1}$.

HRMS (ESI): calculated for $\mathrm{C}_{11} \mathrm{H}_{12} \mathrm{NaO}_{2} \mathrm{~S}^{+}=[\mathrm{M}+\mathrm{Na}]^{+}: \mathrm{m} / \mathrm{z}=231.0450$, found: $\mathrm{m} / \mathrm{z}=$ 231.0453.

\section{(3,3-Diethoxyprop-1ynyl)benzene (1v)}

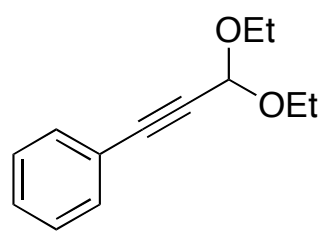

$1 v$

Pale yellow liquid.

${ }^{1} \mathbf{H}$ NMR $\left(\mathrm{CDCl}_{3}, 500 \mathrm{MHz}\right): \delta=1.25(\mathrm{t}, \mathrm{J}=8.5 \mathrm{~Hz}, 6 \mathrm{H}), 3.45-3.60(\mathrm{~m}, 2 \mathrm{H})$, 3.79-3.93 (m, $2 \mathrm{H}), 5.49(\mathrm{~s}, 1 \mathrm{H}), 7.27-7.34(\mathrm{~m}, 3 \mathrm{H}), 7.40-7.51(\mathrm{~m}, 2 \mathrm{H}) \mathrm{ppm}$.

${ }^{13} \mathrm{C} \mathrm{NMR}\left(\mathrm{CDCl}_{3}, 150 \mathrm{MHz}\right): \delta=15.2(2 \mathrm{C}), 61.0(2 \mathrm{C}), 84.4,85.2,91.8,121.9(2 \mathrm{C}), 128.3$, $128.8(2 \mathrm{C}), 132.0 \mathrm{ppm}$.

IR (neat): $v=2927,2237,1489,1354,1327,1093,1042,1006,754,680 \mathrm{~cm}^{-1}$.

HRMS (ESI): calculated for $\mathrm{C}_{13} \mathrm{H}_{16} \mathrm{NaO}_{2}{ }^{+}=[\mathrm{M}+\mathrm{Na}]^{+}: \mathrm{m} / \mathrm{z}=227.1043$, found: $\mathrm{m} / \mathrm{z}=$ 227.1057. 
2,2-Dimethoxy-1,2-diphenylethanone (1z')

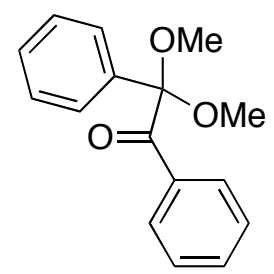

$1 z^{\prime}$

Colorless solid.

${ }^{1} \mathrm{H}$ NMR $\left(\mathrm{CDCl}_{3}, 500 \mathrm{MHz}\right): \delta=3.22(\mathrm{~s}, 6 \mathrm{H}), 7.27-7.33(\mathrm{~m}, 3 \mathrm{H}), 7.33-7.38(\mathrm{~m}, 2 \mathrm{H}), 7.38-$ $7.45(\mathrm{~m}, 1 \mathrm{H}), 7.59-7.65(\mathrm{~m}, 2 \mathrm{H}), 8.03-8.08(\mathrm{~m}, 2 \mathrm{H}) \mathrm{ppm}$.

${ }^{13} \mathrm{C}$ NMR $\left(\mathrm{CDCl}_{3}, 150 \mathrm{MHz}\right): \delta=50.1(2 \mathrm{C}), 103.6,127.0(2 \mathrm{C}), 128.1(2 \mathrm{C}), 128.5(2 \mathrm{C})$, $128.9,130.0(2 \mathrm{C}), 132.9,134.3,136.9,195.2 \mathrm{ppm}$.

IR (neat): $v=2974,1689,1448,1234,1040,1018,866,758,688,659 \mathrm{~cm}^{-1}$.

HRMS: Mass spectroscopic analyses (various techniques) failed to give the molecular signal, resulting only in fragmentation. 


\subsection{Analytical Data for Homoallyl Ethers $\underline{3 a-z '}, \underline{3 a}-\left[d_{2}\right]$, and 3 'a- $\left[d_{2}\right]$}

(1-Methoxybut-3-enyl)benzene $(\underline{3 a})^{12}$<smiles>C=CCC(OC)c1ccccc1</smiles>

$3 \mathbf{a}$

Prepared from acetal 1a $(30.2 \mathrm{mg}, 0.20 \mathrm{mmol})$ and allyl boronic ester 2 (37.0 $\mathrm{mg}, 0.22$ mmol, 1.1 equiv) according to general procedure $A$ using gallium metal $(1.4 \mathrm{mg}, 0.02 \mathrm{mmol}$, $10 \mathrm{~mol} \%)$, silver triflate $(2.8 \mathrm{mg}, 0.01 \mathrm{mmol}, 5 \mathrm{~mol} \%)$, and [18]crown-6 (5.2 mg, $0.02 \mathrm{mmol}$, $10 \mathrm{~mol} \%)$ in dioxane $(200 \mu \mathrm{L}, 1.0 \mathrm{M})$ under ultrasonication at $40-45^{\circ} \mathrm{C}$ for $8 \mathrm{~h}$. 3a was purified by PTLC on silica gel (eluent: heptane/EtOAc = 19:1). The obtained analytical data were in full agreement with the reported data. ${ }^{12}$

Colorless liquid.

Yield: $29.4 \mathrm{mg}$ (91\%).

1-(1-Methoxybut-3-enyl)-4-(trifluoromethyl)benzene (3b)<smiles>C=CCC(OC)c1ccc(C(F)(F)F)cc1</smiles>

$3 \mathbf{b}$

Prepared from acetal 1b $(44.0 \mathrm{mg}, 0.20 \mathrm{mmol})$ and allyl boronic ester 2 (37.0 $\mathrm{mg}, 0.22$ mmol, 1.1 equiv) according to general procedure $A$ using gallium metal $(1.4 \mathrm{mg}, 0.02 \mathrm{mmol}$, $10 \mathrm{~mol} \%)$, silver triflate $(2.8 \mathrm{mg}, 0.01 \mathrm{mmol}, 5 \mathrm{~mol} \%)$, and [18]crown-6 (5.2 mg, $0.02 \mathrm{mmol}$, $10 \mathrm{~mol} \%)$ in dioxane $(200 \mu \mathrm{L}, 1.0 \mathrm{M})$ under ultrasonication at $40-45^{\circ} \mathrm{C}$ for $12 \mathrm{~h}$. $3 \mathrm{~b}$ was purified by PTLC on silica gel (eluent: heptane/EtOAc $=19: 1$ ).

Colorless liquid.

Yield: $42.5 \mathrm{mg}(92 \%)$.

${ }^{1} \mathrm{H}$ NMR $\left(\mathrm{CDCl}_{3}, 500 \mathrm{MHz}\right): \delta=2.39-2.42(\mathrm{~m}, 1 \mathrm{H}), 2.53-2.56(\mathrm{~m}, 1 \mathrm{H}), 3.24(\mathrm{~s}, 3 \mathrm{H}), 4.23$ $(\mathrm{dd}, J=6.3,7.0 \mathrm{~Hz}, 1 \mathrm{H}), 5.03-5.06(\mathrm{~m}, 2 \mathrm{H}), 5.74$ (dddd, $J=6.9,7.0,9.7,17.0 \mathrm{~Hz}, 1 \mathrm{H})$, $7.41(\mathrm{~d}, J=8.1 \mathrm{~Hz}, 2 \mathrm{H}), 7.61(\mathrm{~d}, J=8.1 \mathrm{~Hz}, 2 \mathrm{H}) \mathrm{ppm}$.

${ }^{13} \mathrm{C} \mathrm{NMR}\left(\mathrm{CDCl}_{3}, 125 \mathrm{MHz}\right): \delta=42.3,56.9,83.1,117.5,124.2$ (q, J=271.8 Hz), 125.4 (q, $J=3.8 \mathrm{~Hz}, 2 \mathrm{C}), 127.0(2 \mathrm{C}), 129.9(\mathrm{q}, J=32.4 \mathrm{~Hz}), 134.0,145.9 \mathrm{ppm}$.

${ }^{19} \mathrm{~F} \mathrm{NMR}\left(\mathrm{CDCl}_{3}, 128 \mathrm{MHz}\right): \delta=-62.4 \sim-62.5(\mathrm{~m}) \mathrm{ppm}$.

IR (neat): $v=1320,1163,1122,1097,1064,916,837 \mathrm{~cm}^{-1}$.

HRMS: Mass spectroscopic analyses (various techniques) failed to give the molecular signal, resulting only in fragmentation. 
Methyl 4-(1-methoxybut-3-enyl)benzoate (3c) $)^{14}$<smiles>C=CCC(OC)c1ccc(C(=O)OC)cc1</smiles>

Prepared from acetal 1c $(42.0 \mathrm{mg}, 0.20 \mathrm{mmol})$ and allyl boronic ester $2(37.0 \mathrm{mg}, 0.22$ mmol, 1.1 equiv) according to general procedure $A$ using gallium metal $(1.4 \mathrm{mg}, 0.02 \mathrm{mmol}$, $10 \mathrm{~mol} \%)$, silver triflate (2.8 mg, $0.01 \mathrm{mmol}, 5 \mathrm{~mol} \%)$, and [18]crown-6 (5.2 mg, $0.02 \mathrm{mmol}$, $10 \mathrm{~mol} \%)$ in dioxane $(200 \mu \mathrm{L}, 1.0 \mathrm{M})$ under ultrasonication at $40-45{ }^{\circ} \mathrm{C}$ for $8 \mathrm{~h}$. $3 \mathrm{c}$ was purified by PTLC on silica gel (eluent: heptane/EtOAc $=19: 1$ ). The obtained analytical data were in full agreement with the reported data. ${ }^{14}$

Colorless liquid.

Yield: $40.3 \mathrm{mg}(92 \%)$.

1-Fluoro-4-(1-methoxybut-3-enyl)benzene (3d) $)^{16}$<smiles>C=CCC(OC)c1ccc(F)cc1</smiles>

3d

Prepared from acetal 1d $(34.0 \mathrm{mg}, 0.20 \mathrm{mmol})$ and allyl boronic ester $2(37.0 \mathrm{mg}, 0.22$ mmol, 1.1 equiv) according to general procedure $A$ using gallium metal $(1.4 \mathrm{mg}, 0.02 \mathrm{mmol}$, $10 \mathrm{~mol} \%)$, silver triflate $(2,8 \mathrm{mg}, 0.01 \mathrm{mmol}, 5 \mathrm{~mol} \%)$, and [18]crown-6 $(5.2 \mathrm{mg}, 0.02 \mathrm{mmol}$, $10 \mathrm{~mol} \%)$ in dioxane $(200 \mu \mathrm{L}, 1.0 \mathrm{M})$ under ultrasonication at $40-45{ }^{\circ} \mathrm{C}$ for $8 \mathrm{~h}$. $3 \mathrm{~d}$ was purified by PTLC on silica gel (eluent: heptane/EtOAc $=19: 1$ ). The obtained analytical data were in full agreement with the reported data. ${ }^{16}$

Pale yellow liquid.

Yield: $32.0 \mathrm{mg}(89 \%)$.

\section{1-Chloro-4-(1-methoxybut-3-enyl)benzene (3e) ${ }^{12}$}<smiles>C=CCC(OC)c1ccc(Cl)cc1</smiles>

Prepared from acetal 1 e $(37.2 \mathrm{mg}, 0.20 \mathrm{mmol})$ and allyl boronic ester $2(37.0 \mathrm{mg}, 0.22$ $\mathrm{mmol}, 1.1$ equiv) according to general procedure $A$ using gallium metal $(1.4 \mathrm{mg}, 0.02 \mathrm{mmol}$, $10 \mathrm{~mol} \%$ ), silver triflate (2.8 mg, $0.01 \mathrm{mmol}, 5 \mathrm{~mol} \%)$, and [18]crown-6 (5.2 mg, $0.02 \mathrm{mmol}$, 
$10 \mathrm{~mol} \%)$ in dioxane $(200 \mu \mathrm{L}, 1.0 \mathrm{M})$ under ultrasonication at $40-45{ }^{\circ} \mathrm{C}$ for $12 \mathrm{~h}$. 3e was purified by PTLC on silica gel (eluent: heptane/EtOAc $=19: 1$ ). The obtained analytical data were in full agreement with the reported data. ${ }^{12}$

Colorless liquid.

Yield: $36 \mathrm{mg}(92 \%)$.

1-Bromo-4-(1-methoxybut-3-enyl)benzene (3f) ${ }^{12}$<smiles>C=CCC(OC)c1ccc(Br)cc1</smiles>

Prepared from acetal 1f $(46.2 \mathrm{mg}, 0.20 \mathrm{mmol})$ and allyl boronic ester $2(37.0 \mathrm{mg}, 0.22$ mmol, 1.1 equiv) according to general procedure $A$ using gallium metal $(1.4 \mathrm{mg}, 0.02 \mathrm{mmol}$, $10 \mathrm{~mol} \%)$, silver triflate $(2.8 \mathrm{mg}, 0.01 \mathrm{mmol}, 5 \mathrm{~mol} \%)$, and [18]crown-6 (5.2 mg, $0.02 \mathrm{mmol}$, $10 \mathrm{~mol} \%)$ in dioxane $(200 \mu \mathrm{L}, 1.0 \mathrm{M})$ under ultrasonication at $40-45^{\circ} \mathrm{C}$ for $12 \mathrm{~h}$. 3f was purified by PTLC on silica gel (eluent: heptane/EtOAC $=19: 1$ ). The obtained analytical data were in full agreement with the reported data. ${ }^{12}$

Colorless liquid.

Yield: $44.2 \mathrm{mg}(92 \%)$.

1-(1-Methoxybut-3-enyl)-4-methylbenzene (3g) ${ }^{12}$<smiles>C=CCC(OC)c1ccc(C)cc1</smiles>

$3 g$

Prepared from acetal $\mathbf{1 g}(33.2 \mathrm{mg}, 0.20 \mathrm{mmol})$ and allyl boronic ester $2(37.0 \mathrm{mg}, 0.22$ mmol, 1.1 equiv) according to general procedure $A$ using gallium metal $(1.4 \mathrm{mg}, 0.02 \mathrm{mmol}$, $10 \mathrm{~mol} \%$ ), silver fluoride (1.3 mg, $0.01 \mathrm{mmol}, 5 \mathrm{~mol} \%)$, and [18]crown-6 (5.2 mg, 0.02 $\mathrm{mmol}, 10 \mathrm{~mol} \%)$ in dioxane $(200 \mu \mathrm{L}, 1.0 \mathrm{M})$ under ultrasonication at $40-45^{\circ} \mathrm{C}$ for $20 \mathrm{~h} . \mathbf{3 g}$ was purified by PTLC on silica gel (eluent: heptane/EtOAc $=19: 1$ ). The obtained analytical data were in full agreement with the reported data. ${ }^{12}$

Colorless liquid.

Yield: $33.0 \mathrm{mg}(94 \%)$. 


\section{[4-(1-Methoxybut-3-enyl)phenyl]methanol (3h)}

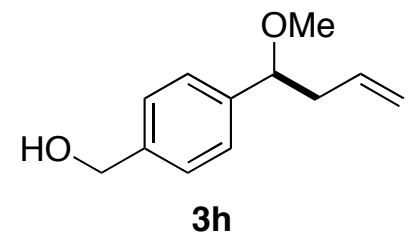

Prepared from acetal $1 \mathrm{~h}(36.4 \mathrm{mg}, 0.20 \mathrm{mmol})$ and allyl boronic ester $2(37.0 \mathrm{mg}, 0.22$ mmol, 1.1 equiv) according to general procedure $A$ using gallium metal $(1.4 \mathrm{mg}, 0.02 \mathrm{mmol}$, $10 \mathrm{~mol} \%)$, silver triflate $(2.8 \mathrm{mg}, 0.01 \mathrm{mmol}, 5 \mathrm{~mol} \%)$, and [18]crown-6 (5.2 mg, $0.02 \mathrm{mmol}$, $10 \mathrm{~mol} \%)$ in toluene $(200 \mu \mathrm{L}, 1.0 \mathrm{M})$ under ultrasonication at $50{ }^{\circ} \mathrm{C}$ for $20 \mathrm{~h}$. 3h was purified by PTLC on silica gel (eluent: heptane/EtOAc = 9:1; eluted twice).

Colorless liquid.

Yield: $20.0 \mathrm{mg}(57 \%)$.

${ }^{1} \mathbf{H}$ NMR $\left(\mathrm{CDCl}_{3}, 400 \mathrm{MHz}\right): \delta=1.64(\mathrm{br} \mathrm{s}, 1 \mathrm{H}), 2.38-2.42(\mathrm{~m}, 1 \mathrm{H}), 2.54-2.59(\mathrm{~m}, 1 \mathrm{H})$, $3.22(\mathrm{~s}, 3 \mathrm{H}), 4.17(\mathrm{dd}, J=6.0,7.3 \mathrm{~Hz}, 1 \mathrm{H}), 4.70(\mathrm{~s}, 2 \mathrm{H}), 5.01-5.08(\mathrm{~m}, 2 \mathrm{H}), 5.71-5.81(\mathrm{~m}$, $1 \mathrm{H}), 7.29(\mathrm{~d}, J=8.1 \mathrm{~Hz}, 2 \mathrm{H}), 7.36(\mathrm{~d}, J=8.1 \mathrm{~Hz}, 2 \mathrm{H}) \mathrm{ppm}$.

${ }^{13} \mathrm{C}$ NMR $\left(\mathrm{CDCl}_{3}, 150 \mathrm{MHz}\right): \delta=42.5,56.7,65.2,83.4,117.0,127.0(2 \mathrm{C}), 127.1$ (2C), $134.7,140.2,141.2 \mathrm{ppm}$.

IR (neat): $v=3421,2862,2849,1463,1095,1016,914,819 \mathrm{~cm}^{-1}$.

HRMS (ESI): calculated for $\mathrm{C}_{12} \mathrm{H}_{16} \mathrm{NaO}_{2}{ }^{+}=[\mathrm{M}+\mathrm{Na}]^{+}: \mathrm{m} / \mathrm{z}=215.1043$, found: $\mathrm{m} / \mathrm{z}=$ 215.1053 .

\section{4-(1-Methoxybut-3-enyl)-N, N-dimethylaniline (3i)}

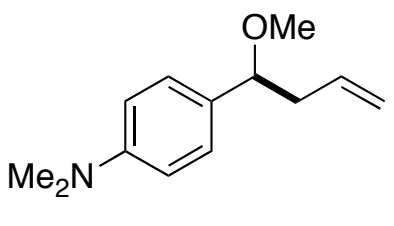

3i

Prepared from acetal 1i $(39.1 \mathrm{mg}, 0.20 \mathrm{mmol})$ and allyl boronic ester 2 (37.0 $\mathrm{mg}, 0.22$ mmol, 1.1 equiv) according to general procedure $A$ using gallium metal $(1.4 \mathrm{mg}, 0.02 \mathrm{mmol}$, $10 \mathrm{~mol} \%)$, silver triflate $(2.8 \mathrm{mg}, 0.01 \mathrm{mmol}, 5 \mathrm{~mol} \%)$, and [18]crown-6 (5.2 mg, $0.02 \mathrm{mmol}$, $10 \mathrm{~mol} \%)$ in toluene $(800 \mu \mathrm{L}, 0.25 \mathrm{M})$ under ultrasonication at $40-45^{\circ} \mathrm{C}$ for $20 \mathrm{~h}$. $3 \mathrm{i}$ was purified by PTLC on silica gel (eluent: heptane/EtOAc $=19: 1$ ).

Pale yellow liquid.

Yield: $22.1 \mathrm{mg}$ (54\%).

${ }^{1} \mathrm{H}$ NMR $\left(\mathrm{CDCl}_{3}, 600 \mathrm{MHz}\right): \delta=2.37-2.42(\mathrm{~m}, 1 \mathrm{H}), 2.54-2.59(\mathrm{~m}, 1 \mathrm{H}), 2.95(\mathrm{~s}, 6 \mathrm{H}), 3.18$ $(\mathrm{s}, 3 \mathrm{H}), 4.07(\mathrm{dd}, J=6.2,7.4 \mathrm{~Hz}, 1 \mathrm{H}), 5.00-5.07(\mathrm{~m}, 2 \mathrm{H}), 5.75-5.80(\mathrm{~m}, 1 \mathrm{H}), 6.71(\mathrm{~d}, J=$ 8.6 Hz, 2H), $7.16(\mathrm{~d}, J=8.6 \mathrm{~Hz}, 2 \mathrm{H}) \mathrm{ppm}$.

${ }^{13} \mathrm{C} \mathrm{NMR}\left(\mathrm{CDCl}_{3}, 150 \mathrm{MHz}\right): \delta=40.6(2 \mathrm{C}), 42.4,56.2,83.3,112.3(2 \mathrm{C}), 116.5,127.8(2 \mathrm{C})$, 129.3, 135.4, $150.2 \mathrm{ppm}$.

IR (neat): $v=2918,2828,1614,1521,1346,1274,1261,1093,912,756 \mathrm{~cm}^{-1}$. 
HRMS (ESI): calculated for $\mathrm{C}_{13} \mathrm{H}_{19} \mathrm{NaNO}^{+}=[\mathrm{M}+\mathrm{Na}]^{+}: \mathrm{m} / z=228.1359$, found: $\mathrm{m} / \mathrm{z}=$ 228.1358.

1-Methoxy-4-(1-methoxybut-3-enyl)benzene $(\underline{3 j})^{12}$<smiles>C=CCC(OC)c1ccc(OC)cc1</smiles>

3j

Prepared from acetal 1j (36.4 mg, $0.20 \mathrm{mmol})$ and allyl boronic acid 2 (37.0 mg, $0.22 \mathrm{mmol}$, 1.1 equiv) according to general procedure $A$ using gallium metal $(1.4 \mathrm{mg}, 0.02 \mathrm{mmol}, 10$ mol\%), silver fluoride (2.8 mg, $0.01 \mathrm{mmol}, 5 \mathrm{~mol} \%$ ), and [18]crown-6 (5.2 mg, $0.02 \mathrm{mmol}$, $10 \mathrm{~mol} \%)$ in dioxane $(200 \mu \mathrm{L}, 1.0 \mathrm{M})$ under ultrasonication at $40-45^{\circ} \mathrm{C}$ for $20 \mathrm{~h}$. $3 \mathrm{j}$ was purified by PTLC on silica gel (eluent: heptane/EtOAC = 19:1). The obtained analytical data were in full agreement with the reported data. ${ }^{12}$

Colorless liquid.

Yield: $35.6 \mathrm{mg}(93 \%)$.

1-(1-Methoxybut-3-enyl)-3-methylbenzene $(\underline{3 k})^{12}$<smiles>C=CCC(OC)c1cccc(C)c1</smiles>

3k

Prepared from acetal 1k $(33.2 \mathrm{mg}, 0.20 \mathrm{mmol})$ and allyl boronic ester $2(37.0 \mathrm{mg}, 0.22$ mmol, 1.1 equiv) according to general procedure $A$ using gallium metal $(1.4 \mathrm{mg}, 0.02 \mathrm{mmol}$, $10 \mathrm{~mol} \%$ ), silver triflate (2.8 mg, $0.01 \mathrm{mmol}, 5 \mathrm{~mol} \%$ ), and [18]crown-6 (5.2 mg, $0.02 \mathrm{mmol}$, $10 \mathrm{~mol} \%)$ in dioxane $(200 \mu \mathrm{L}, 1.0 \mathrm{M})$ under ultrasonication at $40-45^{\circ} \mathrm{C}$ for $12 \mathrm{~h}$. 3k was purified by PTLC on silica gel (eluent: heptane/EtOAc = 19:1). The obtained analytical data were in full agreement with the reported data. ${ }^{12}$

Colorless liquid.

Yield: $32.1 \mathrm{mg}(91 \%)$.

1-(1-Methoxybut-3-enyl)-2-methylbenzene (3I) $)^{12}$<smiles>C=CCC(OC)c1ccccc1C</smiles>

31

Prepared from acetal 11 (33.2 $\mathrm{mg}, 0.20 \mathrm{mmol})$ and allyl boronic ester 2 (37.0 $\mathrm{mg}, 0.22$ mmol, 1.1 equiv) according to general procedure $A$ using gallium metal ( $1.4 \mathrm{mg}, 0.02 \mathrm{mmol}$, 
$10 \mathrm{~mol} \%)$, silver triflate $(2.8 \mathrm{mg}, 0.01 \mathrm{mmol}, 5 \mathrm{~mol} \%)$, and [18]crown-6 $(5.2 \mathrm{mg}, 0.02 \mathrm{mmol}$, $10 \mathrm{~mol} \%)$ in dioxane $(200 \mu \mathrm{L}, 1.0 \mathrm{M})$ under ultrasonication at $40-45{ }^{\circ} \mathrm{C}$ for $8 \mathrm{~h}$. $3 \mathrm{ll}$ was purified by PTLC on silica gel (eluent: heptane/EtOAc $=19: 1$ ). The obtained analytical data were in full agreement with the reported data. ${ }^{12}$

Colorless liquid.

Yield: $32.0 \mathrm{mg}(90 \%)$.

\section{1-(1-Methoxybut-3-enyl)naphthalene $(3 \mathrm{~m})^{12}$}

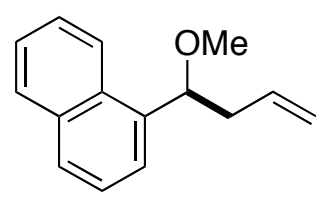

$3 \mathrm{~m}$

Prepared from acetal $1 \mathrm{~m}(40.4 \mathrm{mg}, 0.20 \mathrm{mmol})$ and allyl boronic ester $2(37.0 \mathrm{mg}, 0.22$ mmol, 1.1 equiv) according to general procedure $A$ using gallium metal $(1.4 \mathrm{mg}, 0.02 \mathrm{mmol}$, $10 \mathrm{~mol} \%)$, silver triflate $(2.8 \mathrm{mg}, 0.01 \mathrm{mmol}, 5 \mathrm{~mol} \%)$, and [18]crown-6 (5.2 mg, $0.02 \mathrm{mmol}$, $10 \mathrm{~mol} \%)$ in dioxane $(200 \mu \mathrm{L}, 1.0 \mathrm{M})$ under ultrasonication at $40-45^{\circ} \mathrm{C}$ for $8 \mathrm{~h}$. $3 \mathrm{~m}$ was purified by PTLC on silica gel (eluent: heptane/EtOAc $=19: 1$ ). The obtained analytical data were in full agreement with the reported data. ${ }^{12}$

Colorless liquid.

Yield: $38.5 \mathrm{mg}(91 \%)$.

2-(1-Methoxybut-3-enyl)naphthalene $(3 n)^{12}$<smiles>C=CCC(OC)c1ccc2ccccc2c1</smiles>

3n

Prepared from acetal $1 \mathrm{n}(40.4 \mathrm{mg}, 0.20 \mathrm{mmol})$ and allyl boronic ester $2(37.0 \mathrm{mg}, 0.22$ mmol, 1.1 equiv) according to general procedure $A$ using gallium metal $(1.4 \mathrm{mg}, 0.02 \mathrm{mmol}$, $10 \mathrm{~mol} \%)$, silver triflate $(2.8 \mathrm{mg}, 0.01 \mathrm{mmol}, 5 \mathrm{~mol} \%)$, and [18]crown-6 (5.2 mg, $0.02 \mathrm{mmol}$, $10 \mathrm{~mol} \%)$ in dioxane $(200 \mu \mathrm{L}, 1.0 \mathrm{M})$ under ultrasonication at $40-45{ }^{\circ} \mathrm{C}$ for $8 \mathrm{~h}$. 3n was purified by PTLC on silica gel (eluent: heptane/EtOAc $=19: 1$ ). The obtained analytical data were in full agreement with the reported data. ${ }^{12}$

Colorless liquid.

Yield: $38.1 \mathrm{mg}(91 \%)$. 


\section{(1-Ethoxybut-3-enyl)benzene (30) ${ }^{17}$}<smiles>C=CCC(OCC)c1ccccc1</smiles>

30

Prepared from acetal $10(36.0 \mathrm{mg}, 0.20 \mathrm{mmol})$ and allyl boronic ester $2(37.0 \mathrm{mg}, 0.22$ mmol, 1.1 equiv) according to general procedure $A$ using gallium metal $(1.4 \mathrm{mg}, 0.02 \mathrm{mmol}$, $10 \mathrm{~mol} \%)$, silver triflate $(2.8 \mathrm{mg}, 0.01 \mathrm{mmol}, 5 \mathrm{~mol} \%)$, and [18]crown-6 (5.2 mg, $0.02 \mathrm{mmol}$, $10 \mathrm{~mol} \%)$ in dioxane $(200 \mu \mathrm{L}, 1.0 \mathrm{M})$ under ultrasonication at $40-45^{\circ} \mathrm{C}$ for $12 \mathrm{~h}$. 30 was purified by PTLC on silica gel (eluent: heptane/EtOAC = 19:1). The obtained analytical data were in full agreement with the reported data. ${ }^{17}$

Colorless liquid.

Yield: $31.4 \mathrm{mg}(87 \%)$.

\section{[1-(Benzyloxy)but-3-enyl]benzene $(3 p)^{5,18}$}

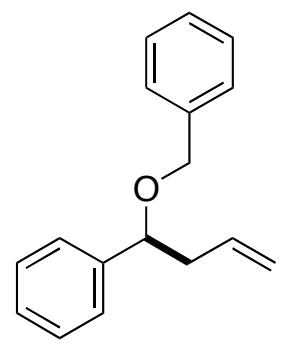

$3 p$

Prepared from acetal $1 \mathrm{p}(54.4 \mathrm{mg}, 0.20 \mathrm{mmol})$ and allyl boronic ester $2(37.0 \mathrm{mg}, 0.22$ mmol, 1.1 equiv) according to general procedure $A$ using gallium metal $(1.4 \mathrm{mg}, 0.02 \mathrm{mmol}$, $10 \mathrm{~mol} \%)$, silver triflate $(2.8 \mathrm{mg}, 0.01 \mathrm{mmol}, 5 \mathrm{~mol} \%)$, and [18]crown-6 (5.2 mg, $0.02 \mathrm{mmol}$, $10 \mathrm{~mol} \%)$ in dioxane $(200 \mu \mathrm{L}, 1.0 \mathrm{M})$ under ultrasonication at $40-45^{\circ} \mathrm{C}$ for $20 \mathrm{~h}$. $3 \mathrm{p}$ was purified by PTLC on silica gel (eluent: heptane/EtOAc = 19:1). The obtained analytical data were in full agreement with the reported data. ${ }^{5,18}$

Colorless liquid.

Yield: $42.0 \mathrm{mg}(88 \%)$.

\section{2-Allyl-2H-chromene $(3 q)^{19}$}

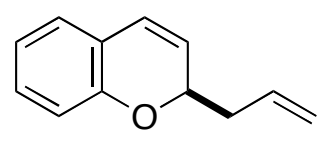

$3 q$

Prepared from acetal 1q $(32.8 \mathrm{mg}, 0.20 \mathrm{mmol})$ and allyl boronic ester $2(37.0 \mathrm{mg}, 0.22$ mmol, 1.1 equiv) according to general procedure $A$ using gallium metal ( $1.4 \mathrm{mg}, 0.02 \mathrm{mmol}$, 
$10 \mathrm{~mol} \%$ ), silver fluoride (1.3 mg, $0.01 \mathrm{mmol}, 5 \mathrm{~mol} \%)$, and [18]crown-6 (5.2 mg, 0.02 $\mathrm{mmol}, 10 \mathrm{~mol} \%)$ in dioxane $(200 \mu \mathrm{L}, 1.0 \mathrm{M})$ under ultrasonication at $40-45^{\circ} \mathrm{C}$ for $52 \mathrm{~h} . \mathbf{3 q}$ was purified by PTLC on silica gel (eluent: heptane/EtOAc = 19:1). The obtained analytical data were in full agreement with the reported data. ${ }^{19}$

Colorless liquid.

Yield: $27.5 \mathrm{mg}(80 \%)$.

\section{1-Allylisochroman $(3 \mathbf{r})^{14}$}

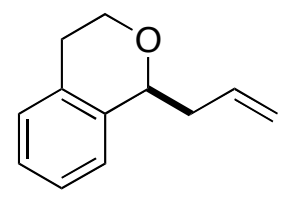

$3 r$

Prepared from acetal $1 \mathrm{r}(32.8 \mathrm{mg}, 0.20 \mathrm{mmol})$ and allyl boronic ester $2(37.0 \mathrm{mg}, 0.22$ mmol, 1.1 equiv) according to general procedure $A$ using gallium metal $(1.4 \mathrm{mg}, 0.02 \mathrm{mmol}$, $10 \mathrm{~mol} \%)$, silver triflate (2.8 mg, $0.01 \mathrm{mmol}, 5 \mathrm{~mol} \%)$, and [18]crown-6 (5.2 mg, $0.02 \mathrm{mmol}$, $10 \mathrm{~mol} \%)$ in dioxane $(200 \mu \mathrm{L}, 1.0 \mathrm{M})$ under ultrasonication at $40-45{ }^{\circ} \mathrm{C}$ for $12 \mathrm{~h}$. $3 \mathrm{r}$ was purified by PTLC on silica gel (eluent: heptane/EtOAc $=19: 1$ ). The obtained analytical data were in full agreement with the reported data. ${ }^{14}$

Colorless liquid.

Yield: $31.2 \mathrm{mg}(90 \%)$.

Tert-butyl-3-(1-methyoxybut-3-enyl)-1 H-indole-1-carboxylate (3s) $)^{14}$<smiles>C=CCC(OC)c1cn(C(=O)OCC(C)(C)C)c2ccccc12</smiles>

Prepared from acetal $1 \mathrm{~s}(58.2 \mathrm{mg}, 0.20 \mathrm{mmol})$ and allyl boronic ester $2(37.0 \mathrm{mg}, 0.22$ mmol, 1.1 equiv) according to general procedure $A$ using gallium metal $(1.4 \mathrm{mg}, 0.02 \mathrm{mmol}$, $10 \mathrm{~mol} \%$ ), silver fluoride (1.3 mg, $0.01 \mathrm{mmol}, 5 \mathrm{~mol} \%$ ), and [18]crown-6 (5.2 mg, 0.02 $\mathrm{mmol}, 10 \mathrm{~mol} \%)$ in toluene $(400 \mu \mathrm{L}, 0.5 \mathrm{M})$ under ultrasonication at $50{ }^{\circ} \mathrm{C}$ for $16 \mathrm{~h}$. 3s was purified by PTLC on silica gel (eluent: heptane/EtOAC $=19: 1$ ). The obtained analytical data were in full agreement with the reported data. ${ }^{14}$

Pale yellow liquid.

Yield: $51.0 \mathrm{mg}(84 \%)$. 


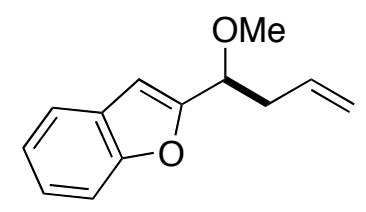

$3 \mathbf{t}$

Prepared from acetal 1t $(38.4 \mathrm{mg}, 0.20 \mathrm{mmol})$ and allyl boronic ester 2 (37.0 $\mathrm{mg}, 0.22$ mmol, 1.1 equiv) according to general procedure $A$ using gallium metal $(1.4 \mathrm{mg}, 0.02 \mathrm{mmol}$, $10 \mathrm{~mol} \%)$, silver fluoride (1.3 mg, $0.01 \mathrm{mmol}, 5 \mathrm{~mol} \%)$, and [18]crown-6 (5.2 mg, 0.02 mmol, $10 \mathrm{~mol} \%)$ in dioxane $(200 \mu \mathrm{L}, 1.0 \mathrm{M})$ under ultrasonication at $40-45{ }^{\circ} \mathrm{C}$ for $20 \mathrm{~h}$. $3 \mathrm{t}$ was purified by PTLC on silica gel (eluent: heptane/EtOAc $=19: 1$ ). The obtained analytical data were in full agreement with the reported data. ${ }^{14}$

Pale yellow liquid.

Yield: $37.0 \mathrm{mg}(90 \%)$.

\section{2-(1-Methoxybut-3-enyl)benzo[b]thiophene (3u)}

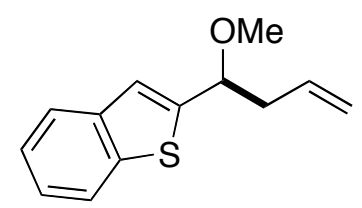

3u

Prepared from acetal $1 \mathbf{u}(41.6 \mathrm{mg}, 0.20 \mathrm{mmol})$ and allyl boronic ester 2 (37.0 mg, 0.22 mmol, 1.1 equiv) according to general procedure $A$ using gallium metal $(1.4 \mathrm{mg}, 0.02 \mathrm{mmol}$, $10 \mathrm{~mol} \%$ ), silver fluoride (1.3 mg, $0.01 \mathrm{mmol}, 5 \mathrm{~mol} \%)$, and [18]crown-6 (5.2 mg, 0.02 $\mathrm{mmol}, 10 \mathrm{~mol} \%)$ in toluene $(200 \mu \mathrm{L}, 1.0 \mathrm{M})$ under ultrasonication at $40-45^{\circ} \mathrm{C}$ for $20 \mathrm{~h}$. $3 \mathrm{u}$ was purified by PTLC on silica gel (eluent: heptane/EtOAc $=19: 1$ ).

Pale yellow liquid.

Yield: $35.0 \mathrm{mg}(80 \%)$.

${ }^{1} \mathrm{H}$ NMR $\left(\mathrm{CDCl}_{3}, 500 \mathrm{MHz}\right): \delta=2.54-2.60(\mathrm{~m}, 1 \mathrm{H}), 2.68-2.75(\mathrm{~m}, 1 \mathrm{H}), 3.32(\mathrm{~s}, 3 \mathrm{H}), 4.49$ $(\mathrm{dd}, J=6.7,6.8 \mathrm{~Hz}, 1 \mathrm{H}), 5.04-5.13(\mathrm{~m}, 2 \mathrm{H}), 5.80$ (dddd, $J=6.9,7.0 .10 .3,17.2 \mathrm{~Hz}, 1 \mathrm{H})$, $7.18(\mathrm{~s}, 1 \mathrm{H}), 7.27-7.35(\mathrm{~m}, 2 \mathrm{H}), 7.72(\mathrm{~d}, J=7.8 \mathrm{~Hz}, 1 \mathrm{H}), 7.82(\mathrm{~d}, J=7.8 \mathrm{~Hz}, 1 \mathrm{H}) \mathrm{ppm}$.

${ }^{13} \mathrm{C}$ NMR $\left(\mathrm{CDCl}_{3}, 150 \mathrm{MHz}\right): \delta=42.3,56.8,79.7,117.6,122.0,122.6,123.4,124.2(2 \mathrm{C})$, 133.9, 139.3, 139.7, 146.5 ppm.

IR (neat): $v=2924,1458,1436,1350,1193,1110,916,827,746,727 \mathrm{~cm}^{-1}$.

HRMS (ESI): calculated for $\mathrm{C}_{13} \mathrm{H}_{14} \mathrm{NaOS}^{+}=[\mathrm{M}+\mathrm{Na}]^{+}: \mathrm{m} / z=241.0658$, found: $\mathrm{m} / z=$ 241.0667. 


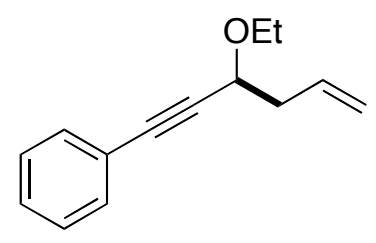

$3 \mathbf{v}$

Prepared from acetal $1 \mathrm{v}(40.9 \mathrm{mg}, 0.20 \mathrm{mmol})$ and allyl boronic ester $2(37.0 \mathrm{mg}, 0.22$ mmol, 1.1 equiv) according to general procedure $A$ using gallium metal $(1.4 \mathrm{mg}, 0.02 \mathrm{mmol}$, $10 \mathrm{~mol} \%)$, silver triflate $(2.8 \mathrm{mg}, 0.01 \mathrm{mmol}, 5 \mathrm{~mol} \%)$, and [18]crown-6 (5.2 mg, $0.02 \mathrm{mmol}$, $10 \mathrm{~mol} \%)$ in dioxane $(200 \mu \mathrm{L}, 1.0 \mathrm{M})$ under ultrasonication at $40-45^{\circ} \mathrm{C}$ for $78 \mathrm{~h}$. $3 \mathbf{v}$ was purified by PTLC on silica gel (eluent: heptane/EtOAC $=19: 1$ ).

Colorless liquid.

Yield: $33.6 \mathrm{mg}(85 \%)$.

${ }^{1} \mathrm{H}$ NMR $\left(\mathrm{CDCl}_{3}, 500 \mathrm{MHz}\right): \delta=1.25(\mathrm{dd}, J=7.0,7.0 \mathrm{~Hz}, 3 \mathrm{H}), 2.53-2.61(\mathrm{~m}, 2 \mathrm{H}), 3.50-$ $3.56(\mathrm{~m}, 1 \mathrm{H}), 3.84-3.90(\mathrm{~m}, 1 \mathrm{H}), 4.29(\mathrm{dd}, J=6.5,6.6 \mathrm{~Hz}, 1 \mathrm{H}), 5.12-5.21(\mathrm{~m}, 2 \mathrm{H}), 5.95$ (dddd, $J=6.9,7.0,10.2,17.2 \mathrm{~Hz}, 1 \mathrm{H}), 7.28-7.31(\mathrm{~m}, 3 \mathrm{H}), 7.42-7.44(\mathrm{~m}, 2 \mathrm{H}) \mathrm{ppm}$.

${ }^{13} \mathrm{C}$ NMR $\left(\mathrm{CDCl}_{3}, 150 \mathrm{MHz}\right): \delta=15.1,40.3,64.3,69.5,85.9,88.1,117.6,122.8,128.2$ (2C), 128.3, $131.8(2 \mathrm{C}), 133.8 \mathrm{ppm}$.

IR (neat): $v=2976,2358,2339,1489,1334,1089,914,760,690 \mathrm{~cm}^{-1}$.

HRMS (ESI): calculated for $\mathrm{C}_{14} \mathrm{H}_{16} \mathrm{NaO}^{+}=[\mathrm{M}+\mathrm{Na}]^{+}: \mathrm{m} / z=223.1093$, found: $\mathrm{m} / z=$ 223.1118.

(E)-(3-Methoxyhexa-1,5-dienyl)benzene $(3 w)^{12}$

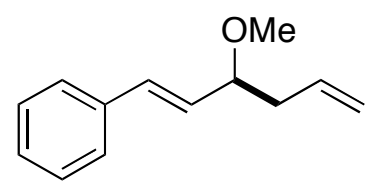

$3 w$

Prepared from acetal $1 \mathrm{w}(35.6 \mathrm{mg}, 0.20 \mathrm{mmol})$ and allyl boronic ester $2(37.0 \mathrm{mg}, 0.22$ mmol, 1.1 equiv) according to general procedure $A$ using gallium metal $(1.4 \mathrm{mg}, 0.02 \mathrm{mmol}$, $10 \mathrm{~mol} \%)$, silver fluoride (1.3 mg, $0.01 \mathrm{mmol}, 5 \mathrm{~mol} \%)$, and [18]crown-6 (5.2 mg, 0.02 $\mathrm{mmol}, 10 \mathrm{~mol} \%)$ in dioxane $(200 \mu \mathrm{L}, 1.0 \mathrm{M})$ under ultrasonication at $50{ }^{\circ} \mathrm{C}$ for $20 \mathrm{~h}$. 3w was purified by PTLC on silica gel (eluent: heptane/EtOAc $=19: 1$ ). The obtained analytical data were in full agreement with the reported data. ${ }^{12}$

Pale yellow liquid.

Yield: $27.0 \mathrm{mg}$ (72\%). 
<smiles>C=CCC(CCc1ccccc1)OC</smiles>

$3 x$

Prepared from acetal $1 \times(36.5 \mathrm{mg}, 0.20 \mathrm{mmol})$ and allyl boronic ester $2(37.0 \mathrm{mg}, 0.22$ mmol, 1.1 equiv) according to general procedure $A$ using gallium metal $(1.4 \mathrm{mg}, 0.02 \mathrm{mmol}$, $10 \mathrm{~mol} \%$ ), silver fluoride (1.3 mg, $0.01 \mathrm{mmol}, 5 \mathrm{~mol} \%)$, and [18]crown-6 (5.2 mg, 0.02 mmol, $10 \mathrm{~mol} \%)$ in dioxane $(200 \mu \mathrm{L}, 1.0 \mathrm{M})$ under ultrasonication at $50{ }^{\circ} \mathrm{C}$ for $20 \mathrm{~h}$. $3 x$ was purified by PTLC on silica gel (eluent: heptane/EtOAc $=19: 1$ ). The obtained analytical data were in full agreement with the reported data. ${ }^{12}$

Pale yellow liquid.

Yield: $32.0 \mathrm{mg}(84 \%)$.

(1-Methoxybut-3-enyl)cyclohexane $(3 y)^{12}$<smiles>C=CCC(OC)C1CCCCC1</smiles>

3y

Prepared from acetal 1y $(31.6 \mathrm{mg}, 0.20 \mathrm{mmol})$ and allyl boronic ester $2(37.0 \mathrm{mg}, 0.22$ mmol, 1.1 equiv) according to general procedure $A$ using gallium metal $(1.4 \mathrm{mg}, 0.02 \mathrm{mmol}$, $10 \mathrm{~mol} \%)$, silver triflate $(2.8 \mathrm{mg}, 0.01 \mathrm{mmol}, 5 \mathrm{~mol} \%)$, and [18]crown-6 (5.2 mg, $0.02 \mathrm{mmol}$, $10 \mathrm{~mol} \%)$ in dioxane $(200 \mu \mathrm{L}, 1.0 \mathrm{M})$ under ultrasonication at $50{ }^{\circ} \mathrm{C}$ for $20 \mathrm{~h}$. $3 \mathrm{y}$ was purified by PTLC on silica gel (eluent: heptane/EtOAC = 19:1). The obtained analytical data were in full agreement with the reported data. ${ }^{12}$

Colorless liquid.

Yield: $26.9 \mathrm{mg}(80 \%)$.

(3-Methoxy-2,2-dimethylhex-5-en-3-yl)benzene (3z)

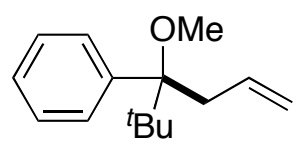

$3 \mathbf{z}$

Prepared from acetal $\mathbf{1 z}(41.6 \mathrm{mg}, 0.20 \mathrm{mmol})$ and allyl boronic ester 2 (37.0 $\mathrm{mg}, 0.22$ mmol, 1.1 equiv) according to general procedure $A$ using gallium metal $(1.4 \mathrm{mg}, 0.02 \mathrm{mmol}$, $10 \mathrm{~mol} \%)$, silver triflate $(2.8 \mathrm{mg}, 0.01 \mathrm{mmol}, 5 \mathrm{~mol} \%)$, and [18]crown-6 (5.2 mg, $0.02 \mathrm{mmol}$, $10 \mathrm{~mol} \%)$ in dioxane $(200 \mu \mathrm{L}, 1.0 \mathrm{M})$ under ultrasonication at $50{ }^{\circ} \mathrm{C}$ for $40 \mathrm{~h} . \mathbf{3 z}$ was purified by PTLC on silica gel (eluent: heptane/EtOAc = 19:1). 
Colorless liquid.

Yield: $39.8 \mathrm{mg}(91 \%)$.

${ }^{1} \mathbf{H}$ NMR $\left(\mathrm{CDCl}_{3}, 500 \mathrm{MHz}\right): \delta=0.88(\mathrm{~s}, 9 \mathrm{H}), 2.94-3.03(\mathrm{~m}, 2 \mathrm{H}), 3.22(\mathrm{~s}, 3 \mathrm{H}), 4.97$ (ddd, J $=2.2,3.4,10.2 \mathrm{~Hz}, 1 \mathrm{H}), 5.13(\mathrm{ddd}, J=2.2,3.4,17.2 \mathrm{~Hz}, 1 \mathrm{H}), 5.93-6.00(\mathrm{~m}, 1 \mathrm{H}), 7.21-$ $7.32(\mathrm{~m}, 5 \mathrm{H}) \mathrm{ppm}$.

${ }^{13} \mathrm{C}$ NMR $\left(\mathrm{CDCl}_{3}, 150 \mathrm{MHz}\right): \delta=26.5(3 \mathrm{C}), 37.0,39.5,52.6,85.3,115.3,126.2,126.8(2 \mathrm{C})$, 129.3 (2C), 137.3. $140.8 \mathrm{ppm}$.

IR (neat): $v=2958,1483,1444,1392,1363,1097,1074,906,748,705 \mathrm{~cm}^{-1}$.

HRMS (ESI): calculated for $\mathrm{C}_{15} \mathrm{H}_{22} \mathrm{NaO}^{+}=[\mathrm{M}+\mathrm{Na}]^{+}: \mathrm{m} / z=241.1563$, found: $m / z=241.1557$.

2-Methoxy-1,2-diphenylpent-4-en-1-one (3z')

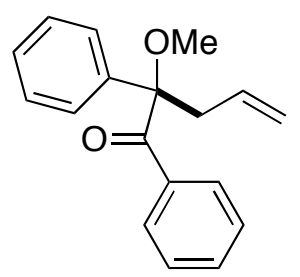

$3 z^{\prime}$

Prepared from acetal 1z' $(51.3 \mathrm{mg}, 0.20 \mathrm{mmol})$ and allyl boronic ester 2 (37.0 mg, 0.22 mmol, 1.1 equiv) according to general procedure $A$ using gallium metal (1.4 mg, $0.02 \mathrm{mmol}$, $10 \mathrm{~mol} \%$ ), silver fluoride (1.3 mg, $0.01 \mathrm{mmol}, 5 \mathrm{~mol} \%)$, and [18]crown-6 (5.2 mg, 0.02 $\mathrm{mmol}, 10 \mathrm{~mol} \%)$ in dioxane $(200 \mu \mathrm{L}, 1.0 \mathrm{M})$ under ultrasonication at $40-45^{\circ} \mathrm{C}$ for $60 \mathrm{~h}$. $3 \mathrm{z}^{\prime}$ was purified by PTLC on silica gel (eluent: heptane/EtOAc $=19: 1$ ).

Colorless liquid.

Yield: $43.6 \mathrm{mg}(82 \%)$.

${ }^{1} \mathrm{H}$ NMR $\left(\mathrm{CDCl}_{3}, 500 \mathrm{MHz}\right): \delta=3.08(\mathrm{~s}, 3 \mathrm{H}), 3.41-3.43(\mathrm{~m}, 2 \mathrm{H}), 5.00$ (ddd, $J=1.6,3.1$, $17.2 \mathrm{~Hz}, 1 \mathrm{H}$ ), 5.08 (ddd, $J=1.6,3.1,10.2 \mathrm{~Hz}, 1 \mathrm{H}$ ), 5.83 (dddd, $J=6.8,6.9,10.2,17.2 \mathrm{~Hz}$, $1 \mathrm{H}), 7.26-7.42(\mathrm{~m}, 10 \mathrm{H}) \mathrm{ppm}$.

${ }^{13} \mathrm{C}$ NMR $\left(\mathrm{CDCl}_{3}, 150 \mathrm{MHz}\right): \delta=42.4,53.0,91.4,118.2,128.0,128.1$ (6C), 129.0 (4C), 131.1, 138.7, 208.3 ppm.

IR (neat): $v=2916,2356,2341,1718,1490,1446,1066,914,738,680 \mathrm{~cm}^{-1}$.

HRMS (ESI): calculated for $\mathrm{C}_{18} \mathrm{H}_{18} \mathrm{NaO}_{2}{ }^{+}=[\mathrm{M}+\mathrm{Na}]^{+}: \mathrm{m} / z=289.1199$, found: $\mathrm{m} / z=$ 289.1212. 


\section{(4,4-Dideuterio-1-methoxybut-3-enyl)benzene $\left(3 a-\left[d_{2}\right]\right)^{14} /$}

(2,2-Dideuterio-1-methoxybut-3-enyl)benzene (3'a- $\left.\left[d_{2}\right]\right)^{14}$

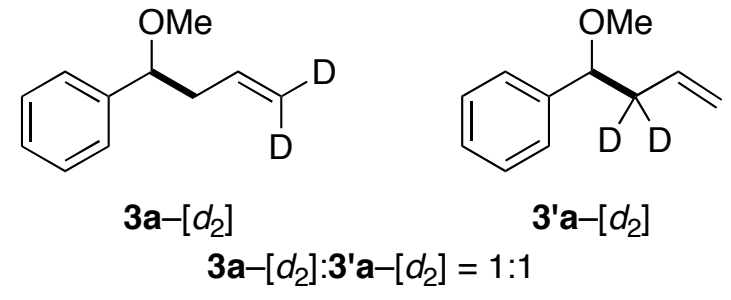

Prepared from acetal 1a $(30.4 \mathrm{mg}, 0.20 \mathrm{mmol})$ and allyl boronic ester 2-[ $\left.d_{2}\right](51.0 \mathrm{mg}, 0.30$ mmol, 1.5 equiv) according to general procedure $A$ using gallium metal $(1.4 \mathrm{mg}, 0.02 \mathrm{mmol}$, $10 \mathrm{~mol} \%)$, silver triflate $(2.8 \mathrm{mg}, 0.01 \mathrm{mmol}, 5 \mathrm{~mol} \%)$, and [18]crown-6 (5.2 mg, $0.02 \mathrm{mmol}$, $10 \mathrm{~mol} \%)$ in dioxane $(200 \mu \mathrm{L}, 1.0 \mathrm{M})$ under ultrasonication at $40-45^{\circ} \mathrm{C}$ for $8 \mathrm{~h} .3$ 'a- $\left[d_{2}\right]$ and 3'a-[ $\left[\boldsymbol{d}_{2}\right]$ were purified by PTLC on silica gel (eluent: heptane/EtOAc $=19: 1$ ). The obtained analytical data were in full agreement with the reported data. ${ }^{14}$

Pale yellow liquid.

Yield: $\left.26.0 \mathrm{mg}\left\{80 \% ; \mathbf{3} \mathbf{a}-\left[d_{2}\right]: \mathbf{3}^{\prime} \mathbf{a}-\left[d_{2}\right]=1: 1\right]\right\}$. 


\subsection{Analytical Data for Homopropargyl Ethers 15a and 15x}

(1-Methoxybut-3-ynyl)benzene $(15 a)^{20}$<smiles>C#CCC(OC)c1ccccc1</smiles>

$15 a$<smiles>C=C=CC(OC)c1ccccc1</smiles>

$16 a$

$15 a: 16 a=49: 1$

Prepared from acetal 1a (30.4 mg, $0.20 \mathrm{mmol})$ and allenyl boronic ester 14 (39.9 mg, 0.24 mmol, 1.2 equiv) according to general procedure $A$ using gallium metal $(1.4 \mathrm{mg}, 0.02 \mathrm{mmol}$, $10 \mathrm{~mol} \%$ ), silver fluoride (1.3 mg, $0.01 \mathrm{mmol}, 5 \mathrm{~mol} \%)$, and [18]crown-6 (5.2 mg, 0.02 $\mathrm{mmol}, 10 \mathrm{~mol} \%)$ in dioxane $(200 \mu \mathrm{L}, 1.0 \mathrm{M})$ under ultrasonication at $40-45{ }^{\circ} \mathrm{C}$ for $52 \mathrm{~h}$. 15a and 16a were purified by PTLC on silica gel (eluent: heptane/EtOAc = 19:1). The obtained analytical data were in full agreement with the reported data. ${ }^{20}$

Pale yellow liquid.

Yield: $29.0 \mathrm{mg}(91 \% ; 15 a: 16 a=49: 1)$.

(3-Methoxyhex-5-ynyl)benzene $(15 x)^{21,22}$<smiles>C#CCC(CCc1ccccc1)OC</smiles>

$15 x$<smiles>C=C=CC(CCc1ccccc1)OC</smiles>

$16 x$

$15 x: 16 x=>30: 1$

Prepared from acetal 1x (36.4 mg, $0.20 \mathrm{mmol})$ and allenyl boronic ester 14 (39.9 mg, 0.24 mmol, 1.2 equiv) according to general procedure $A$ using gallium metal ( $1.4 \mathrm{mg}, 0.02 \mathrm{mmol}$, $10 \mathrm{~mol} \%$ ), silver fluoride (1.3 mg, $0.01 \mathrm{mmol}, 5 \mathrm{~mol} \%)$, and [18]crown-6 (5.2 mg, 0.02 $\mathrm{mmol}, 10 \mathrm{~mol} \%)$ in dioxane $(200 \mu \mathrm{L}, 1.0 \mathrm{M})$ under ultrasonication at $40-45{ }^{\circ} \mathrm{C}$ for $72 \mathrm{~h}$. 15x and 16x were purified by PTLC on silica gel (eluent: heptane/EtOAc = 19:1). The obtained analytical data were in full agreement with the reported data. ${ }^{21,22}$

Colorless liquid.

Yield: $31.1 \mathrm{mg}(82 \% ; 15 x: 16 x=>30: 1)$. 


\subsection{Analytical Data for Silver Phosphate $(R)-17^{23}$}<smiles>CC(C)(C)OP(=O)(O)Oc1c(-c2cc(C(C)(C)C)cc(C(C)(C)C)c2)cc2ccccc2c1-c1c(OP(=O)(OC(C)(C)C)OC(C)(C)C)cc(C(C)(C)C)cc1-c1cc(C(C)(C)C)cc(C(C)(C)C)c1</smiles>

(R)-17

Silver phosphate $(R)-17$ was obtained from the corresponding enantiopure phosphoric acid and silver carbonate $\left(\mathrm{Ag}_{2} \mathrm{CO}_{3}\right)$ according to a literature report. ${ }^{24}$ The phosphoric acid was prepared from the enantiopure diol and phosphoryl chloride $\left(\mathrm{POCl}_{3}\right)$ according to a literature report. $^{25}(R)-\mathbf{1 7}$ was purified by filtration over activated celite, followed by rigorous drying in vacuo. The obtained analytical data were in full agreement with the reported data. ${ }^{23}$

Colorless solid. 


\subsection{Analytical Data for Homoallyl Amides rac-13 and $(R)-13$}

\section{Rac-N-(1-Phenylbut-3-enyl)benzamide (rac-13) $)^{23}$}

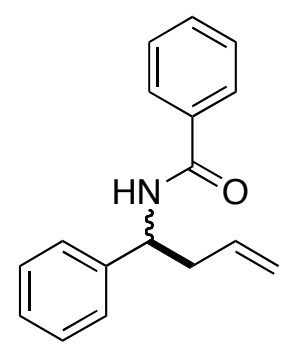

rac-13

Prepared from aminal rac-12 $(48.3 \mathrm{mg}, 0.20 \mathrm{mmol})$ and allyl boronic ester $2(40.0 \mathrm{mg}, 0.24$ mmol, 1.2 equiv) according to general procedure $A$ using gallium metal $(1.4 \mathrm{mg}, 0.02 \mathrm{mmol}$, $10 \mathrm{~mol} \%$ ), silver triflate $(1.3 \mathrm{mg}, 0.01 \mathrm{mmol}, 5 \mathrm{~mol} \%)$, and 18 [crown]-6 $(5.2 \mathrm{mg}, 0.02 \mathrm{mmol}$, $10 \mathrm{~mol} \%)$ in dioxane $(200 \mu \mathrm{L}, 1.0 \mathrm{M})$ at $50{ }^{\circ} \mathrm{C}$ for $24 \mathrm{~h}$. Rac-13 was purified by PTLC on silica gel [eluent: $\mathrm{PE}(40-60) / \mathrm{Et}_{2} \mathrm{O}=5: 1$; eluted three times]. The obtained analytical data were in full agreement with the reported data. ${ }^{23}$

Colorless solid.

Yield: $41.6 \mathrm{mg}(83 \%)$.

(R)-N-(1-Phenylbut-3-enyl)benzamide $[(R)-13]^{23}$

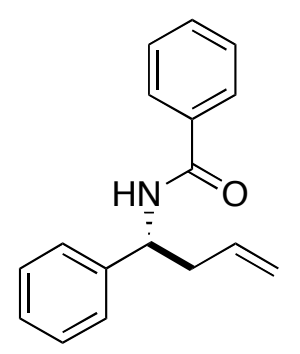

$(R)-13$

Prepared from aminal rac-12 $(48.3 \mathrm{mg}, 0.20 \mathrm{mmol})$ and allyl boronic ester $2(50.4 \mathrm{mg}, 0.30$ mmol, 1.5 equiv), according to general procedure $C$, using gallium metal $(2.1 \mathrm{mg}, 0.03$ mmol, $15 \mathrm{~mol} \%)$ and silver salt $(R)-17(8.3 \mathrm{mg}, 0.015 \mathrm{mmol}, 7.5 \mathrm{~mol} \%)$, in toluene $(200 \mu \mathrm{L}$, $1.0 \mathrm{M}$ ) at $40^{\circ} \mathrm{C}$ for $5 \mathrm{~d}$. (R)-13 was purified by PTLC on silica gel [eluent: PE (40-60)/Et $\mathrm{t}_{2} \mathrm{O}$ $=5: 1$; eluted four times]. The obtained analytical data were in full agreement with the reported data. ${ }^{23}$

Colorless solid.

Yield: $30.1 \mathrm{mg}(60 \%) ; 40 \%$ ee.

HPLC (DAICEL CHIRALPAK IF; eluent: hexane/'PrOH = 9:1; flow rate: $1.0 \mathrm{~mL} / \mathrm{min}$ ): $t_{r}=$ $15.4(R), 21.0(S)$ min.

The absolute configuration of 13 was assigned in analogy to literature. ${ }^{23}$ 
(1) Roush, W. R.; Adam, M. A.; Walts, A. E.; Harris, D. J. J. Am. Chem. Soc. 1986, 108, 3422.

(2) Sieber, J. D.; Morken, J. P. J. Am. Chem. Soc. 2008, 130, 4978.

(3) Challinor, A. J.; Calin, M.; Nichol, G. S.; Carter, N. B.; Thomas, S. P. Adv. Synth. Catal. 2016, 15, 2404.

(4) Williams, D. B. G.; Lawton, M. C. Green Chem. 2008, 10, 914.

(5) Madabhushi, S.; Mallu, K. K. R.; Chinthala, N.; Beeram, C. R.; Vangipuram, V. S. Tetrahedron Lett. 2012, 53, 697.

(6) Moquist, P. N.; Kodama, T.; Schaus, S. E. Angew. Chem., Int. Ed. 2010, 49, 7096.

(7) Reisman, S. E.; Doyle, A. G.; Jacobsen, E. N. J. Am. Chem. Soc. 2008, 130, 7198.

(8) Katritzky, A. R.; Fan, W.-Q.; Black, M.; Pernak, J. J. Org. Chem. 1992, 52, 547.

(9) Fujioka, H.; Goto, A.; Otake, K.; Kubo, O.; Sawama, Y.; Maegawa, T. Chem. Commun. 2011, 47, 9894.

(10) Wiles, C.; Watts, P.; Haswell, S. J. Tetrahedron 2005, 61, 5209.

(11) Sakai, N.; Moritaka, K.; Konakahara, T. Eur. J. Org. Chem. 2009, 2009, 4123.

(12) Ito, S.; Hayashi, A.; Komai, H.; Yamaguchi, H.; Kubota, Y.; Asami, M. Tetrahedron 2011, 67, 2081.

(13) Kumar, D.; Kumar, R.; Chakraborti, A. K. Synthesis 2008, 1249.

(14) Schneider, U.; Dao, H. T.; Kobayashi, S. Org. Lett. 2010, 12, 2488.

(15) Ortholand, J.-Y.; Vicart, N.; Greiner, A. J. Org. Chem. 1995, 60, 1880.

(16) Merisor, E.; Conrad, J.; Malakar, C. C.; Beifuss, U. Synlett 2008, 903.

(17) Yang, M.-S.; Xu, L.-W.; Zhang, F.-B.; Qiu, H.-Y.; Jiang, J.-X.; Lai, G.-Q. Appl. Organomet. Chem. 2008, 22, 177.

(18) Watahiki, T.; Akabane, Y.; Mori, S.; Oriyama, T. Org. Lett. 2003, 5, 3045.

(19) Clausen, D. J.; Floreancig, P. E. J. Org. Chem. 2012, 77, 6574.

(20) Niimi, L.; Hiraoka, S.; Yokozawa, T. Tetrahedron 2002, 58, 245.

(21) Motodate, S.; Kobayashi, T.; Fujii, M.; Mochida, T.; Kusakabe, T.; Katoh, S.; Akita, H.; Kato, K. Chem. - Asian J. 2010, 5, 2221.

(22) Pragani, R.; Roush, W. R. Org. Lett. 2008, 10, 4613.

(23) Huang, Y.-Y.; Chakrabarti, A.; Morita, N.; Schneider, U.; Kobayashi, S. Angew. Chem., Int. Ed. 2011, 50, 11121.

(24) Hamilton, G. L.; Kang, E. J.; Mba, M.; Toste, F. D. Science 2007, 317, 496.

(25) Liu, W.-J.; Chen, X.-H.; Gong, L.-Z. Org. Lett. 2008, 10, 5357. 


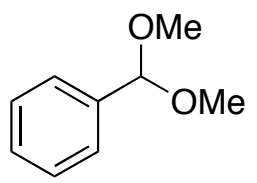

$1 \mathrm{a}$
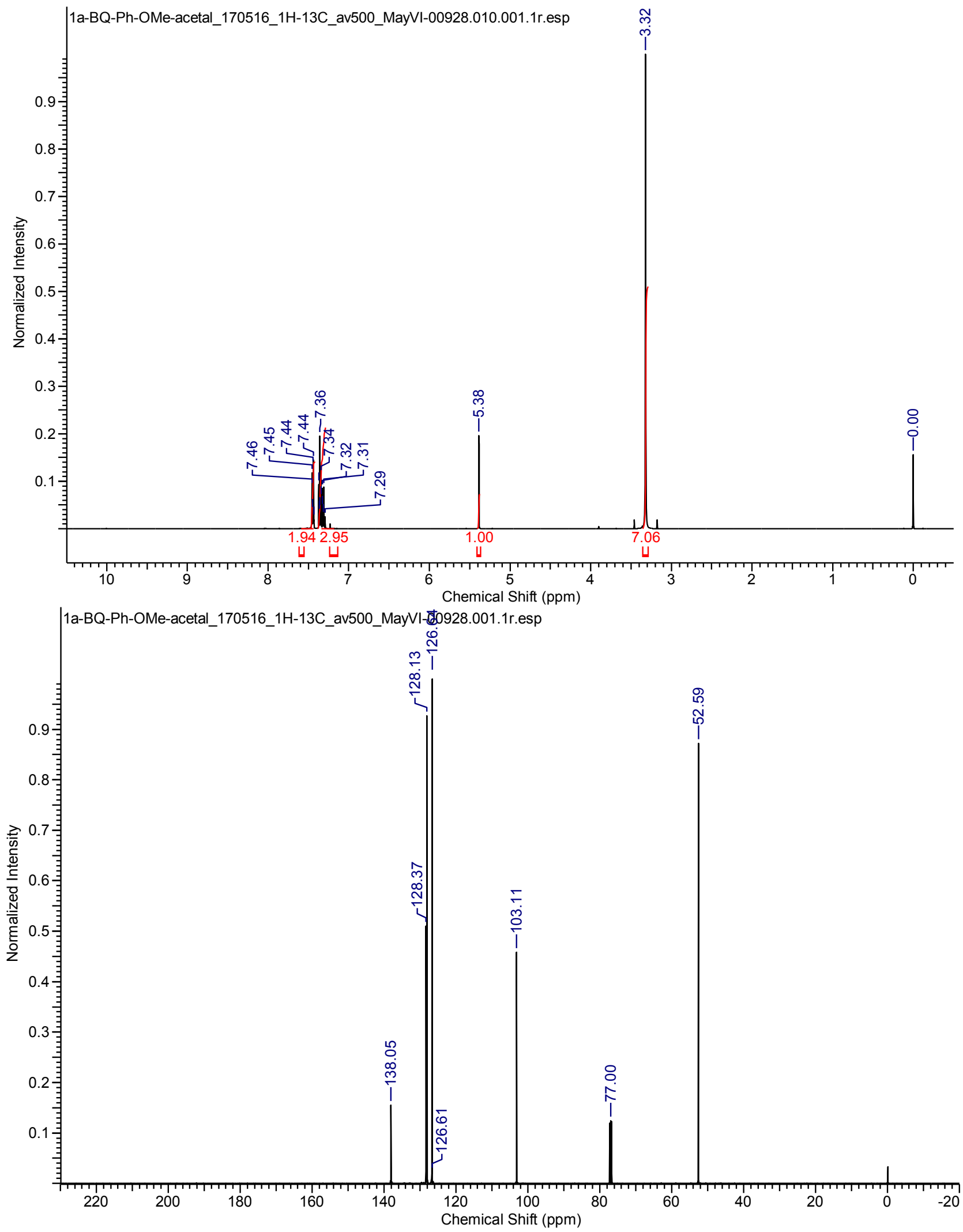
<smiles>COC(OC)c1ccc(C(F)(F)F)cc1</smiles>

$1 \mathrm{~b}$
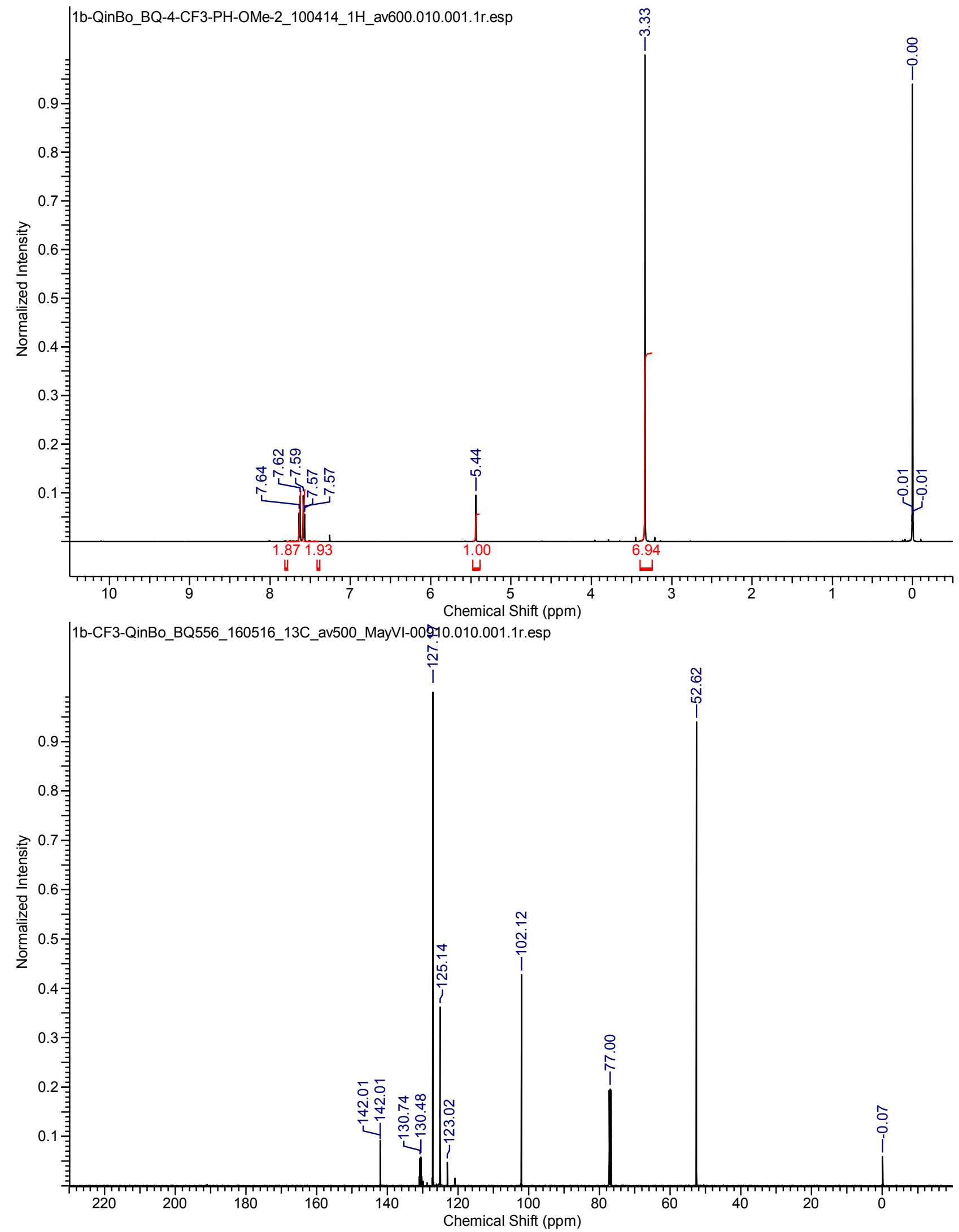


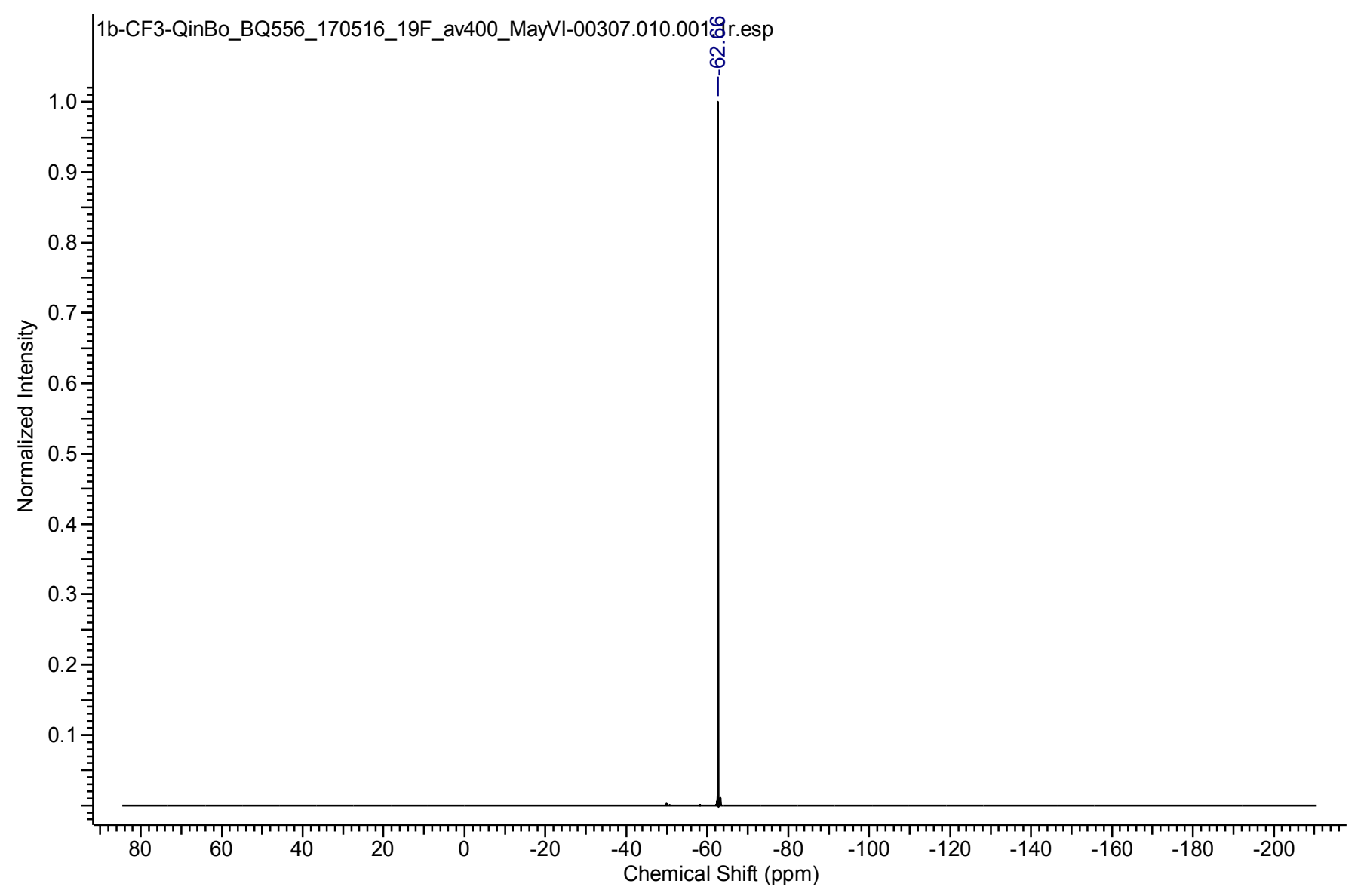




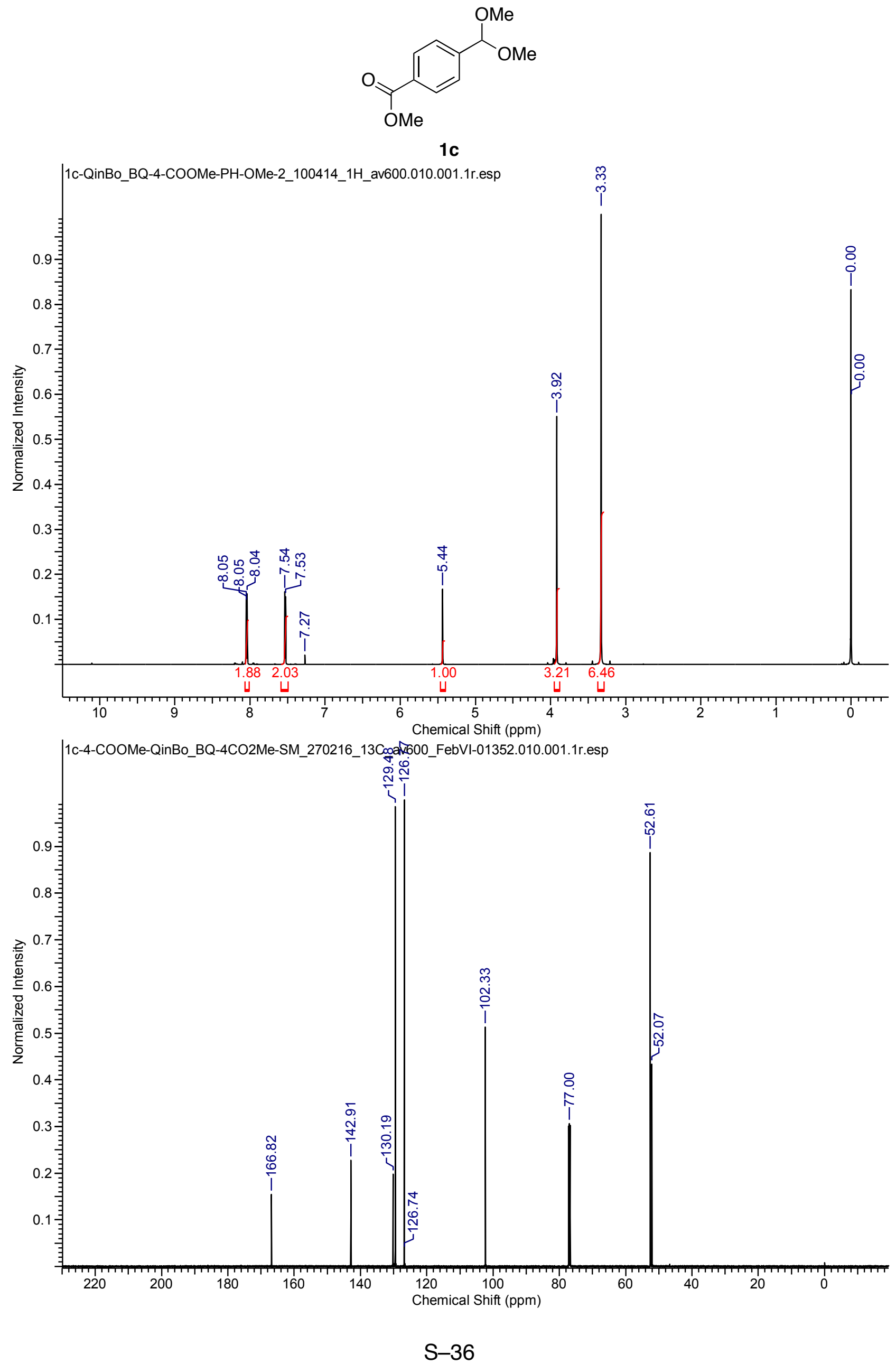



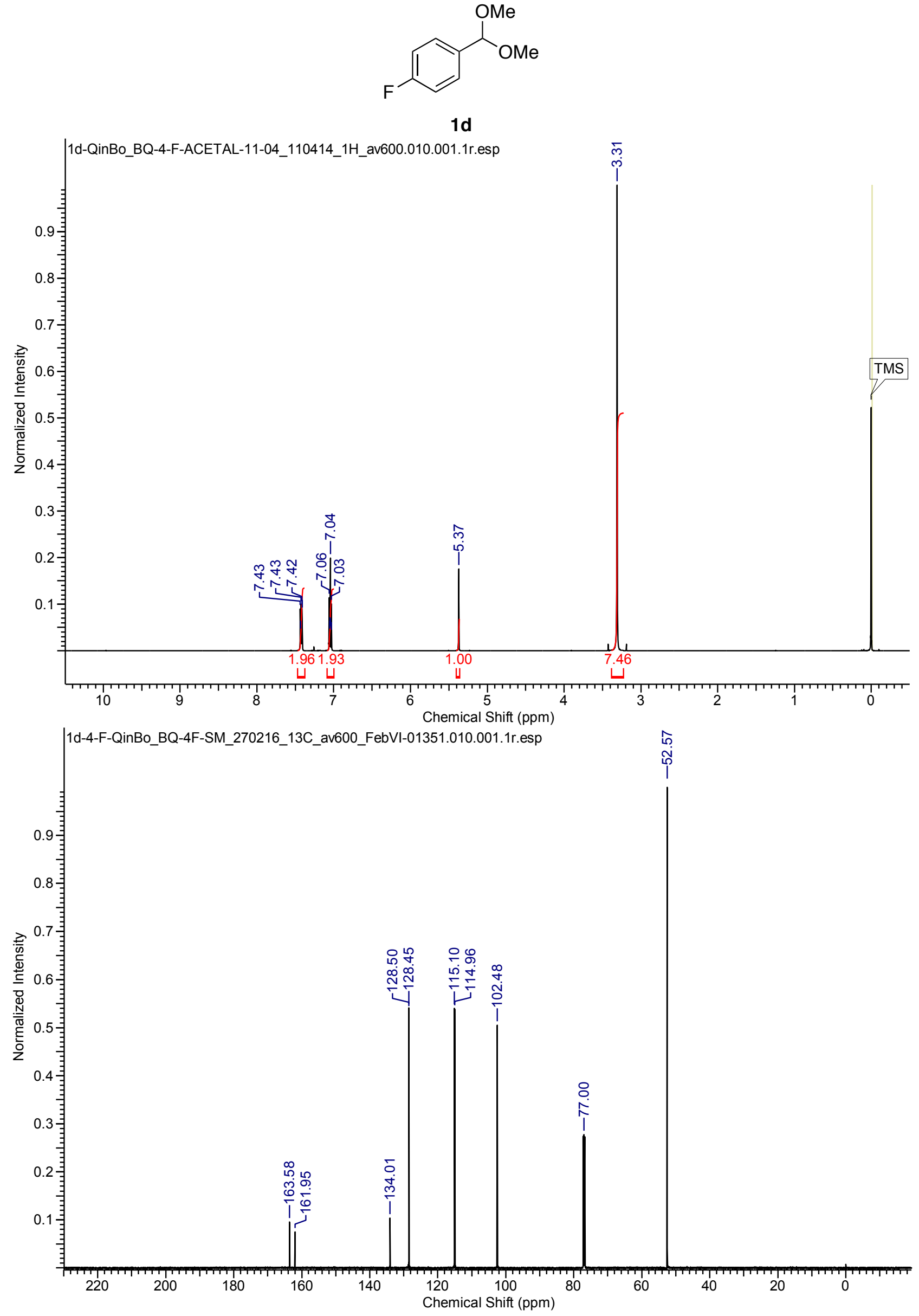


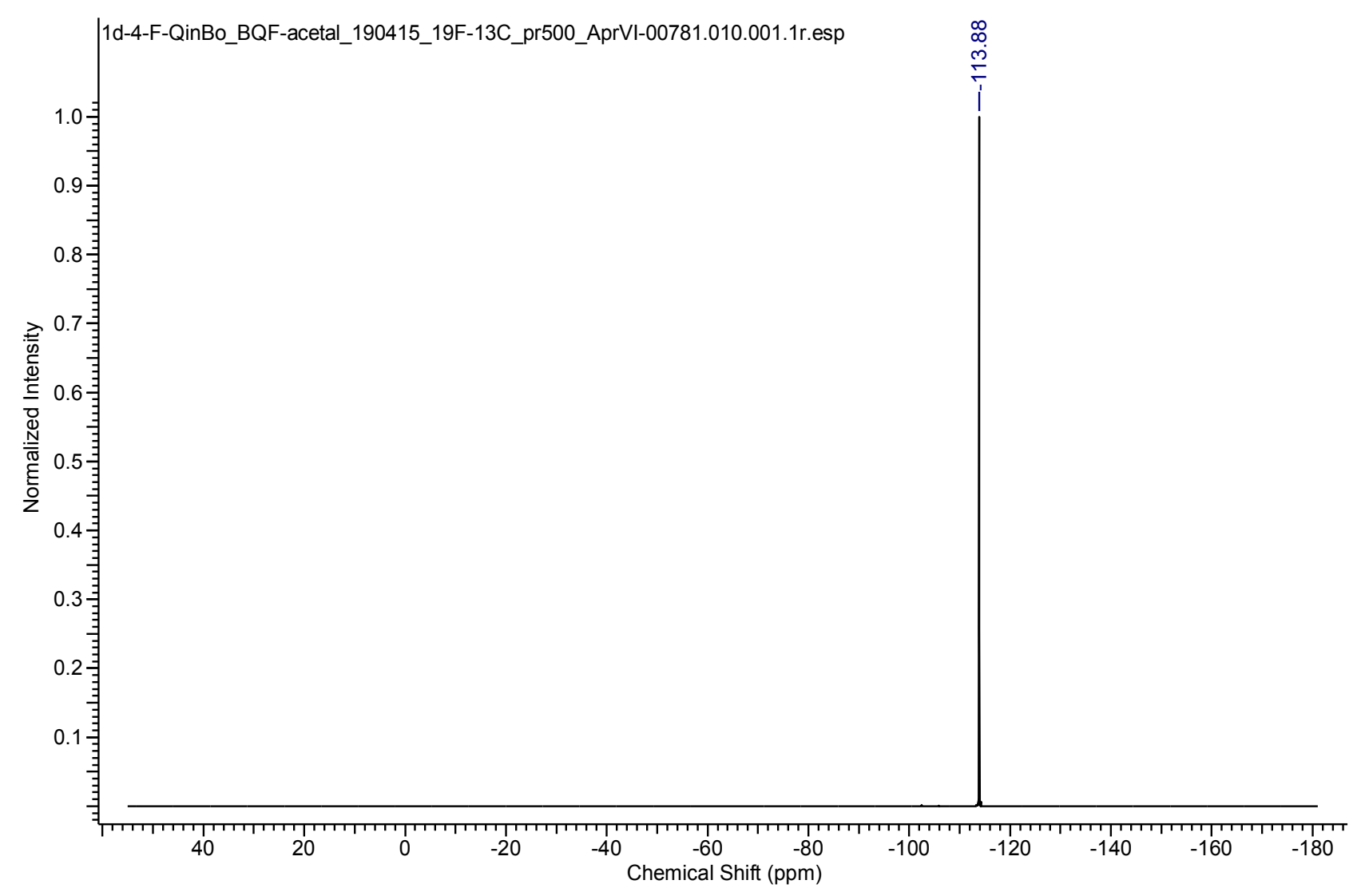



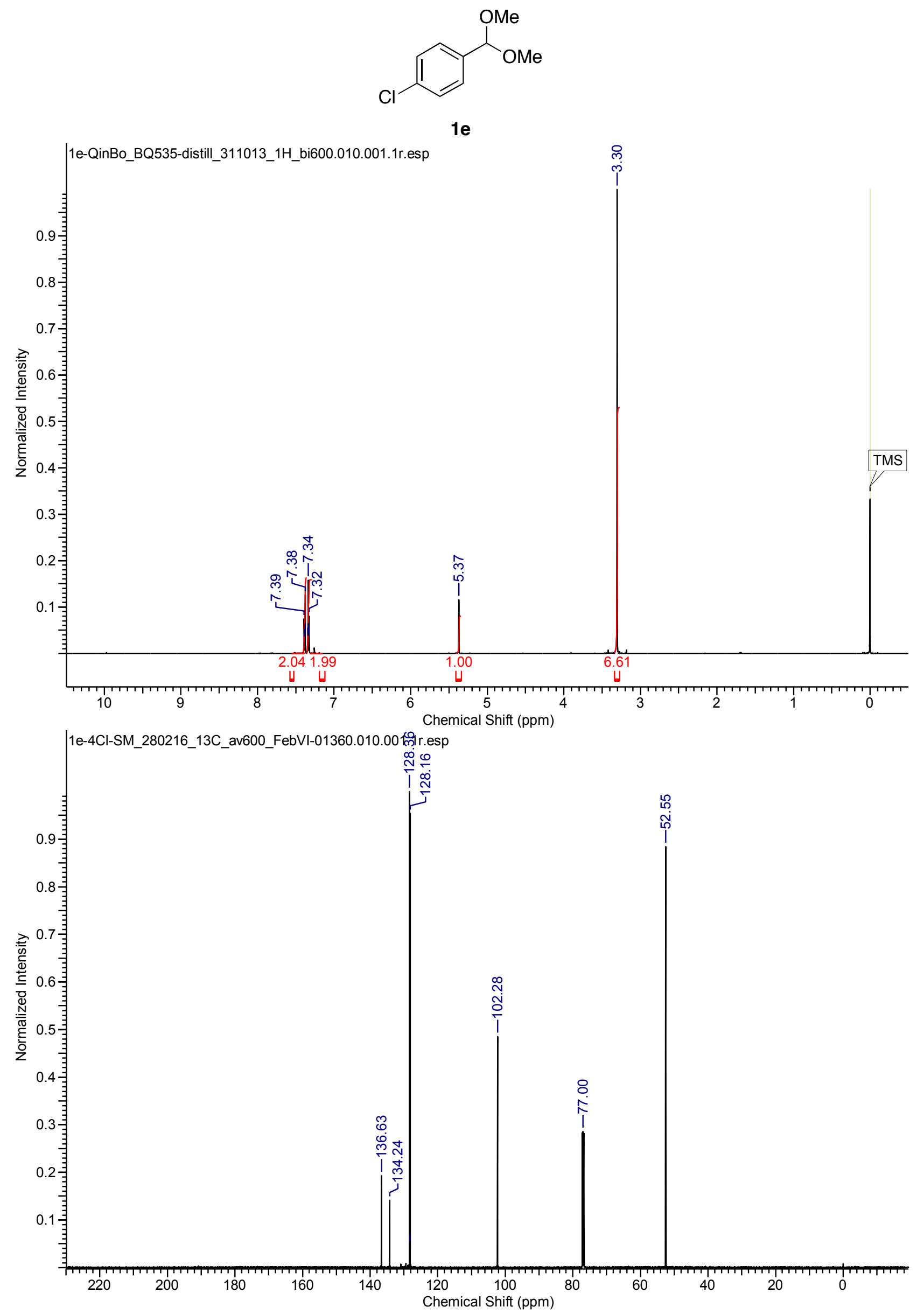

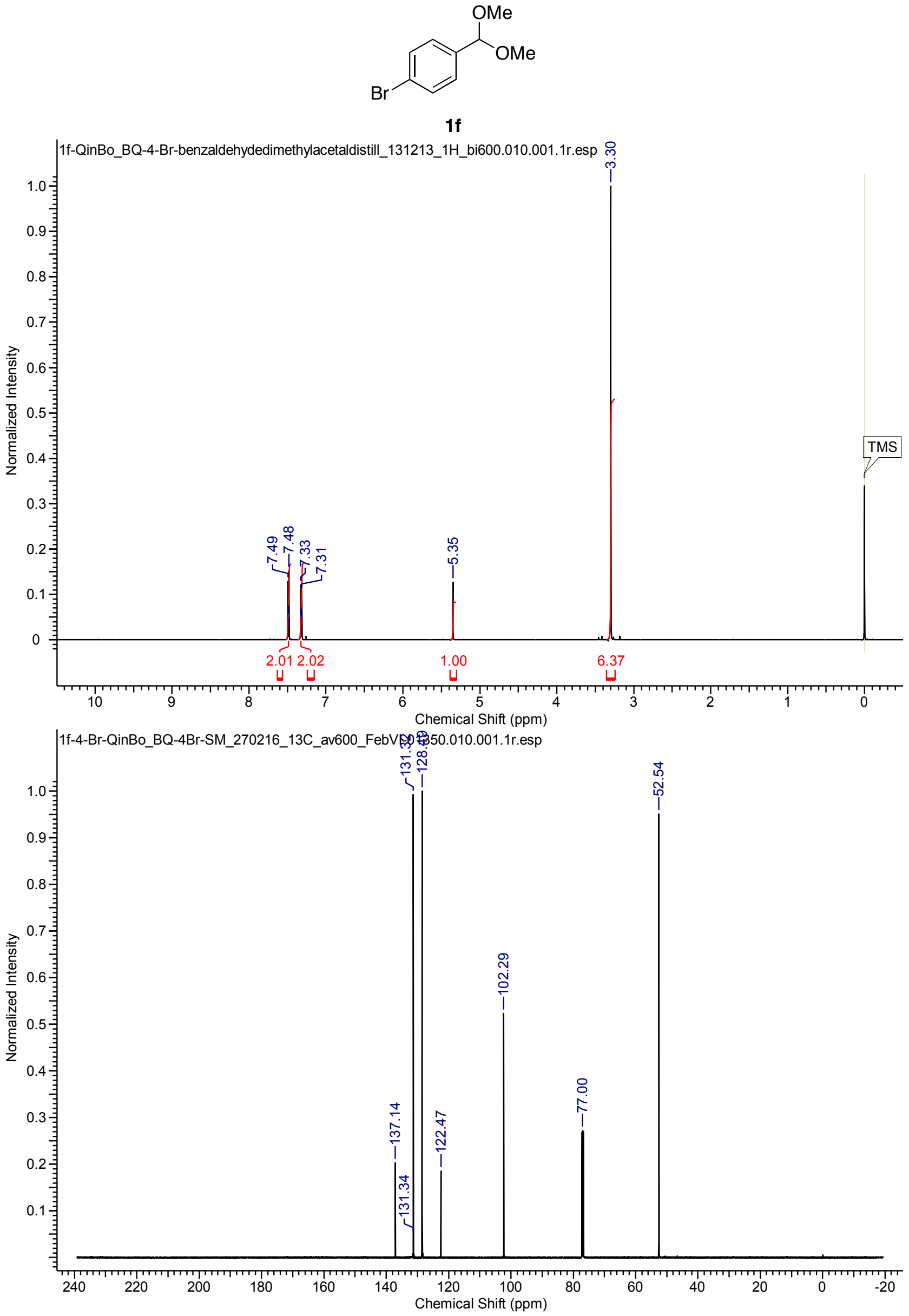
<smiles>COC(OC)c1ccc(C)cc1</smiles>

$1 \mathrm{~g}$
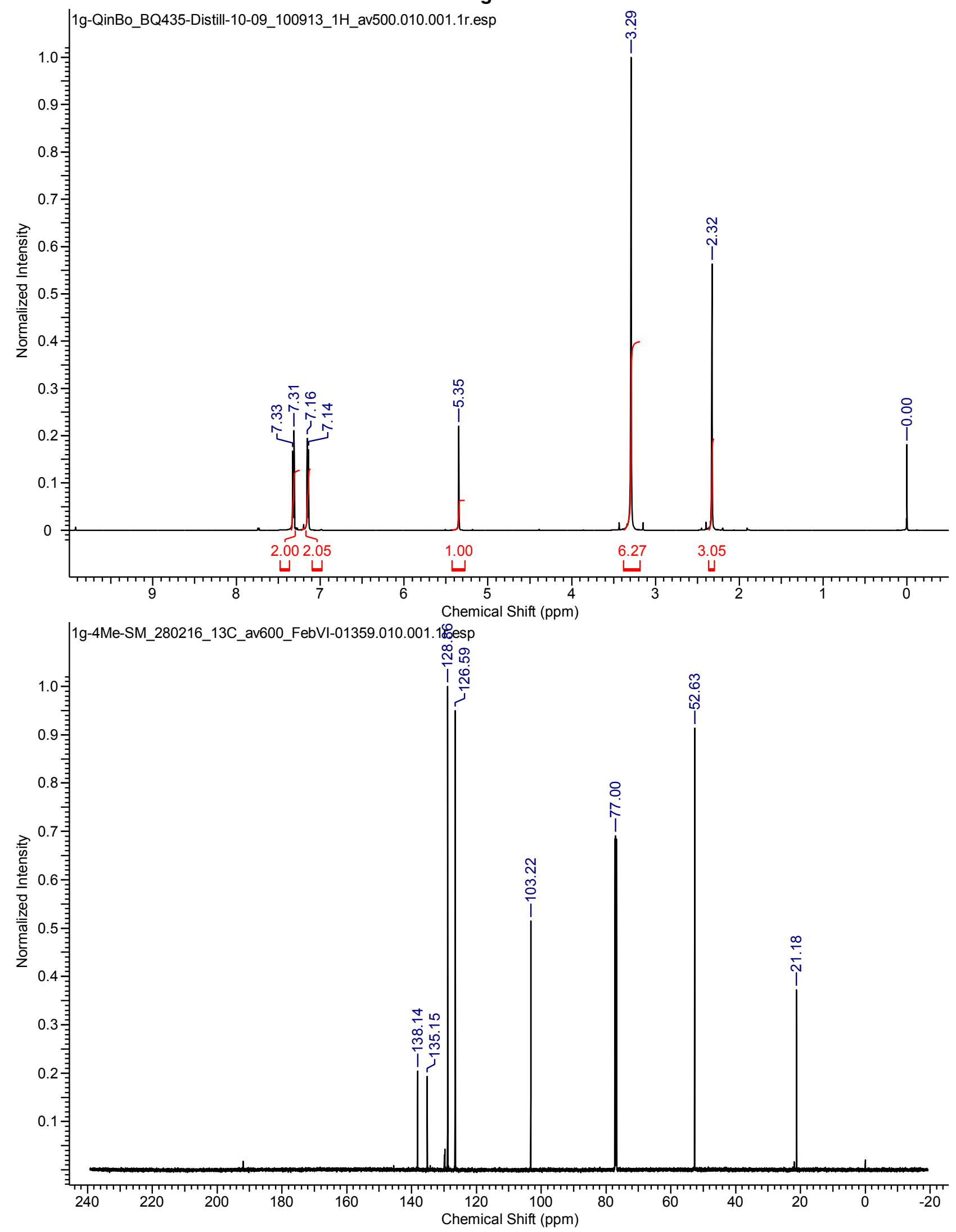
<smiles>COC(OC)c1ccc(CO)cc1</smiles>

$1 \mathrm{~h}$
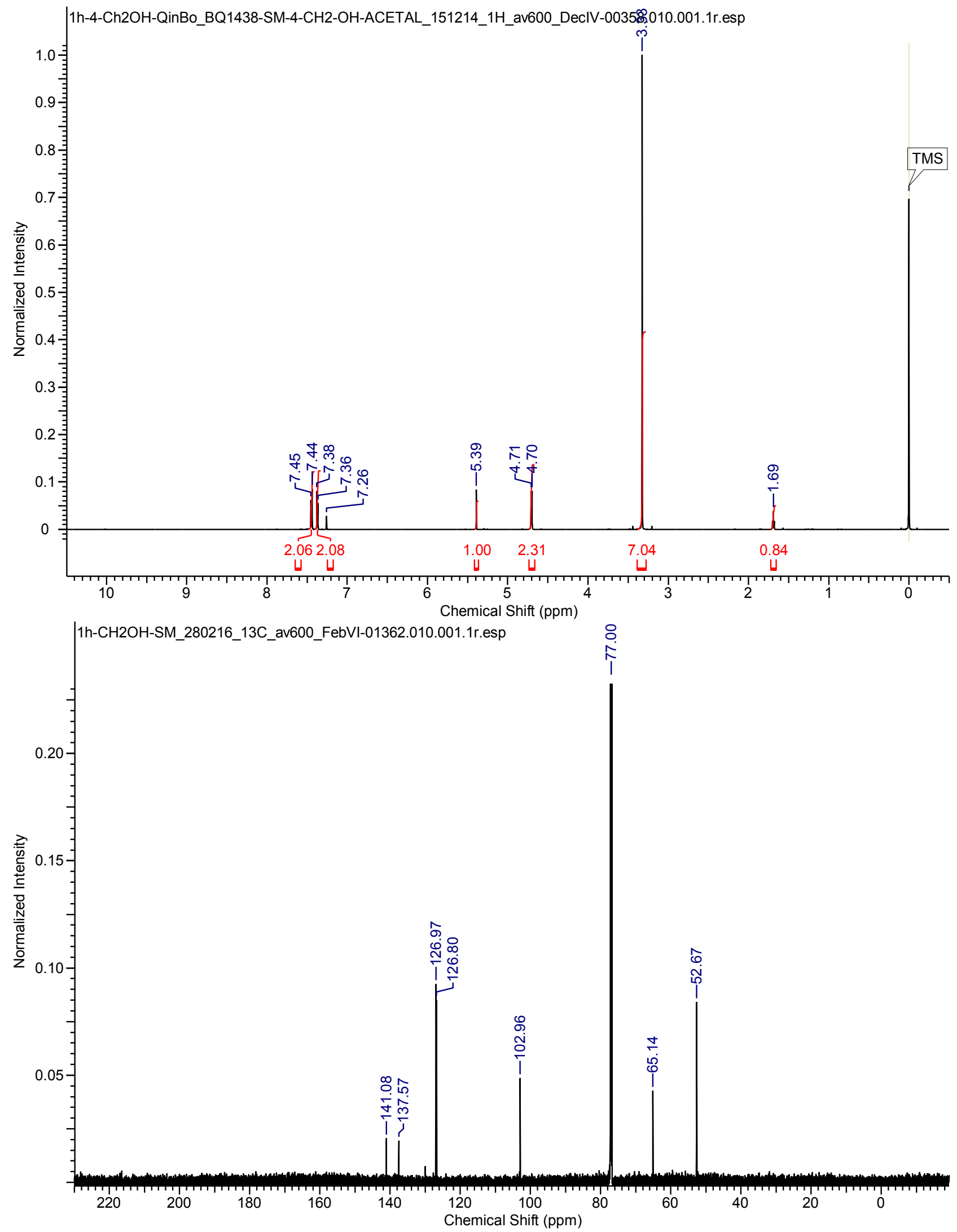
<smiles>COC(OC)c1ccc(N(C)C)cc1</smiles>

$1 \mathrm{i}$
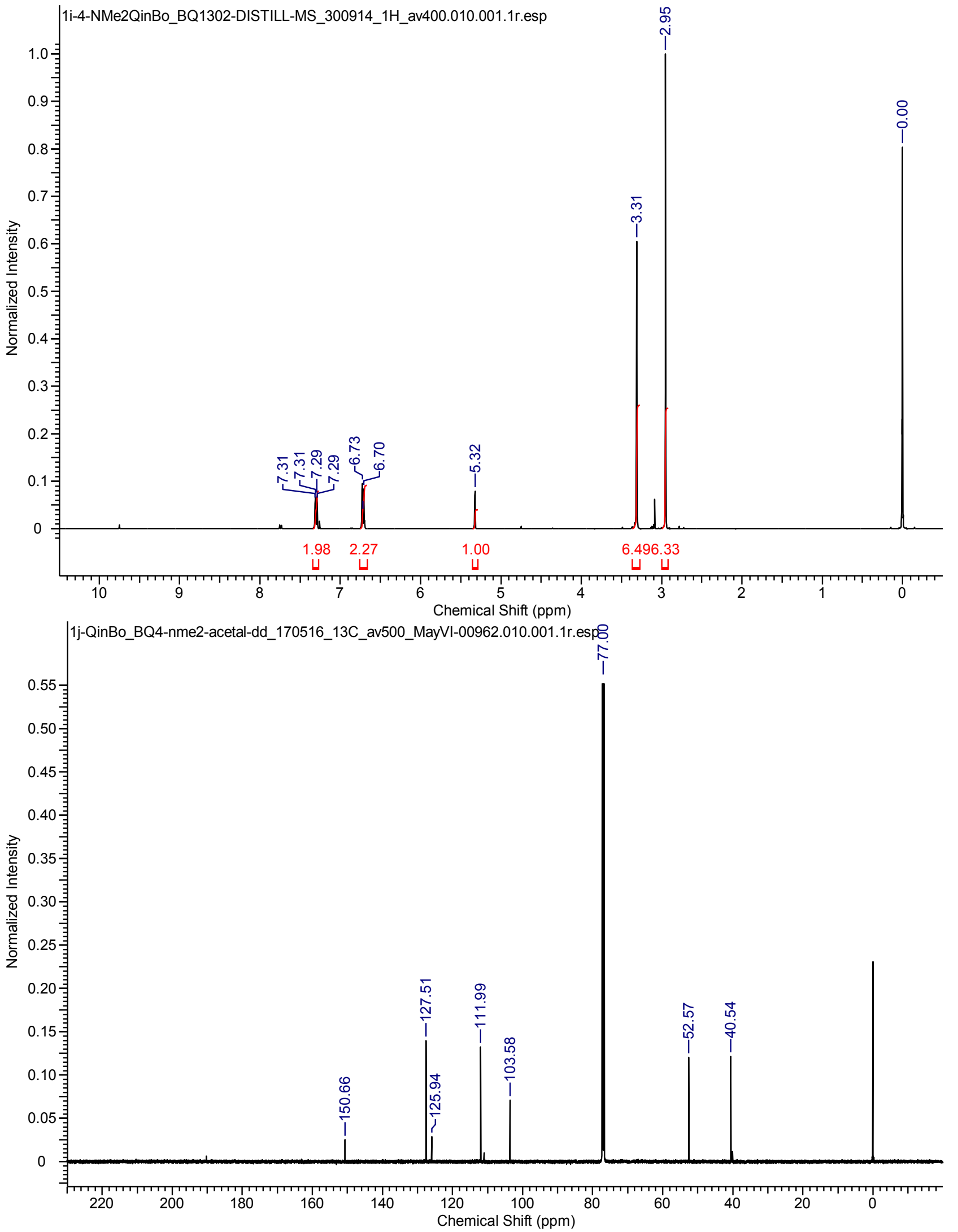


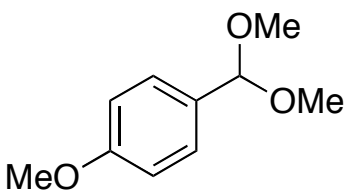

1j
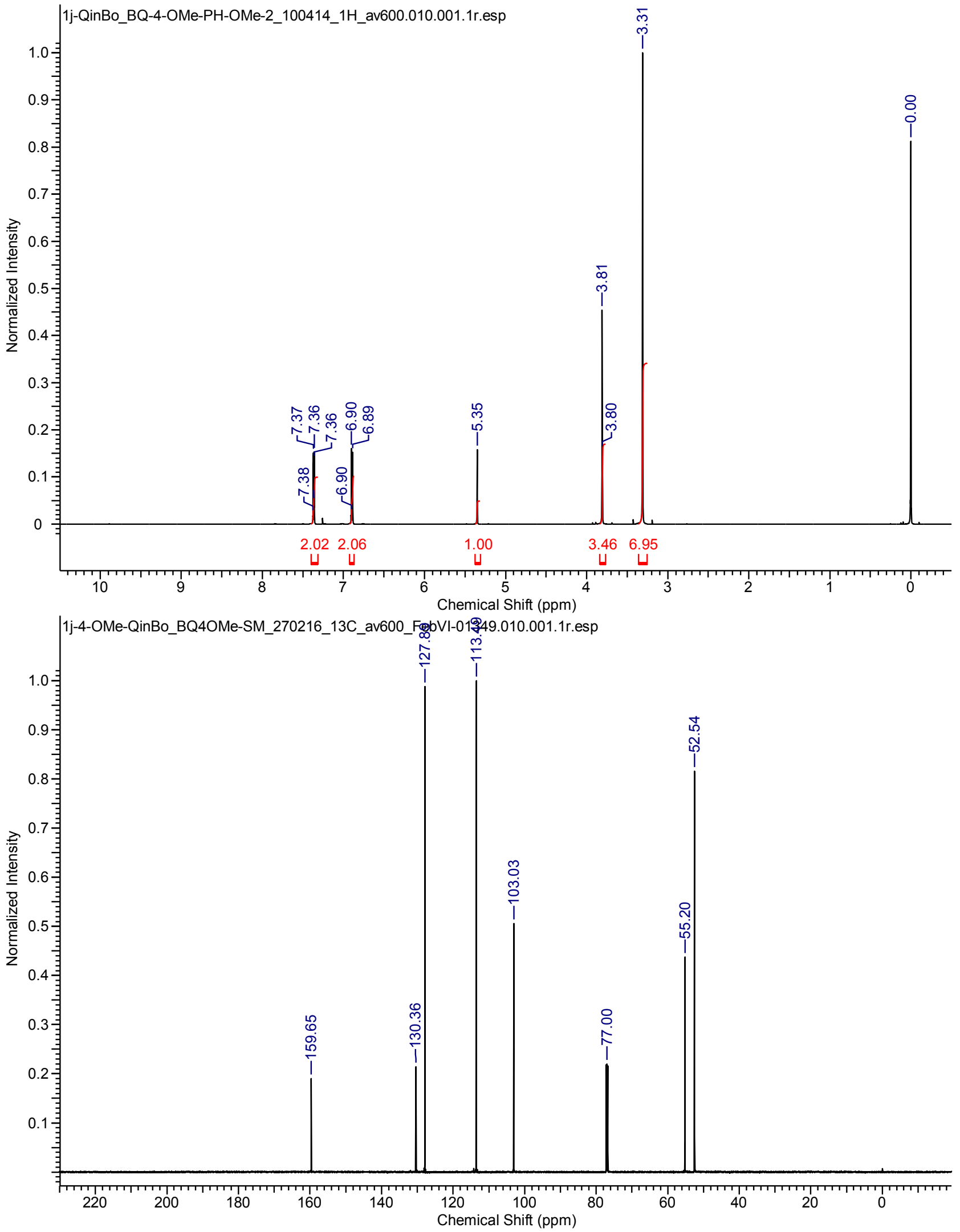
<smiles>COC(OC)c1cccc(C)c1</smiles>

$1 \mathrm{k}$
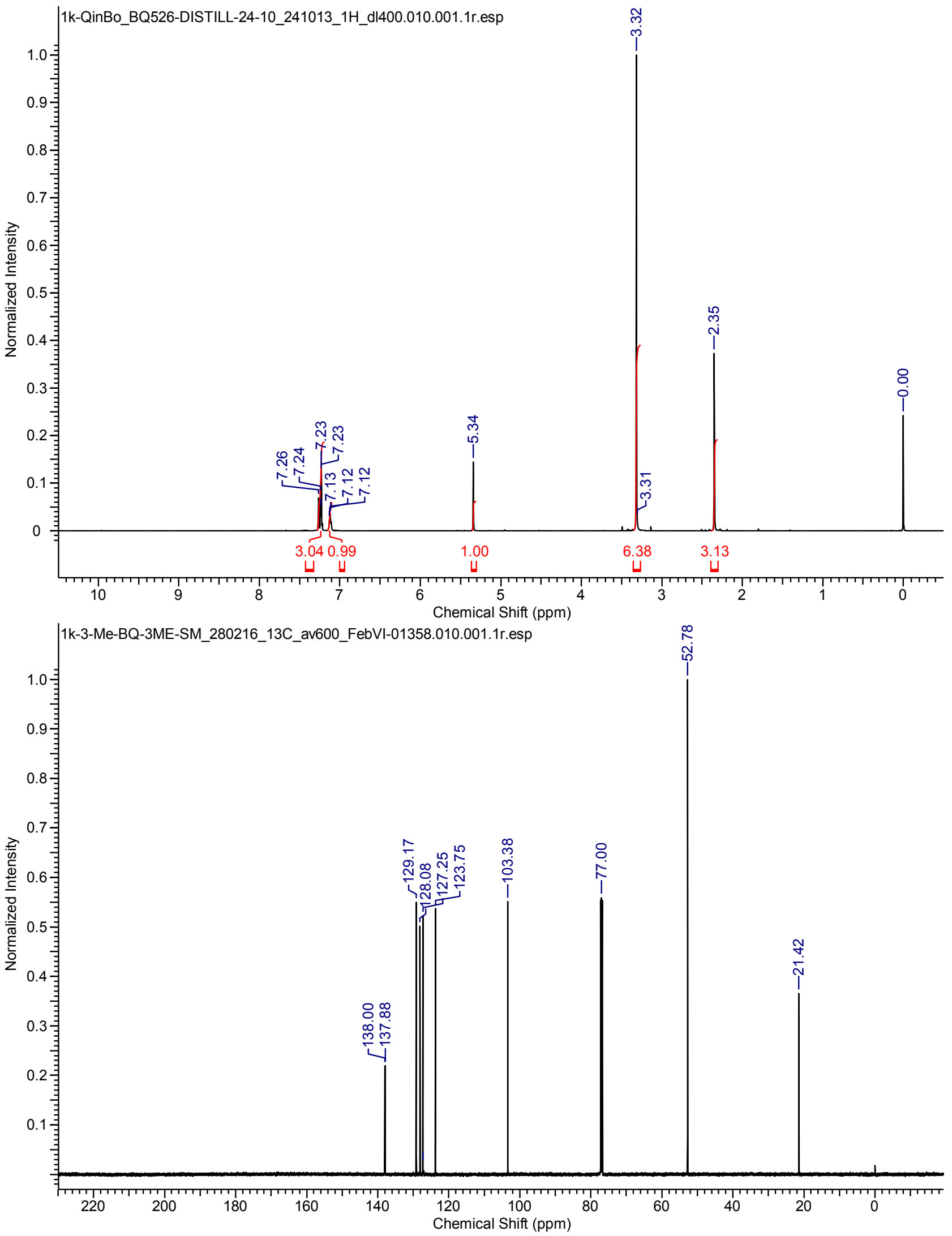


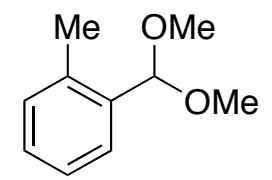

11
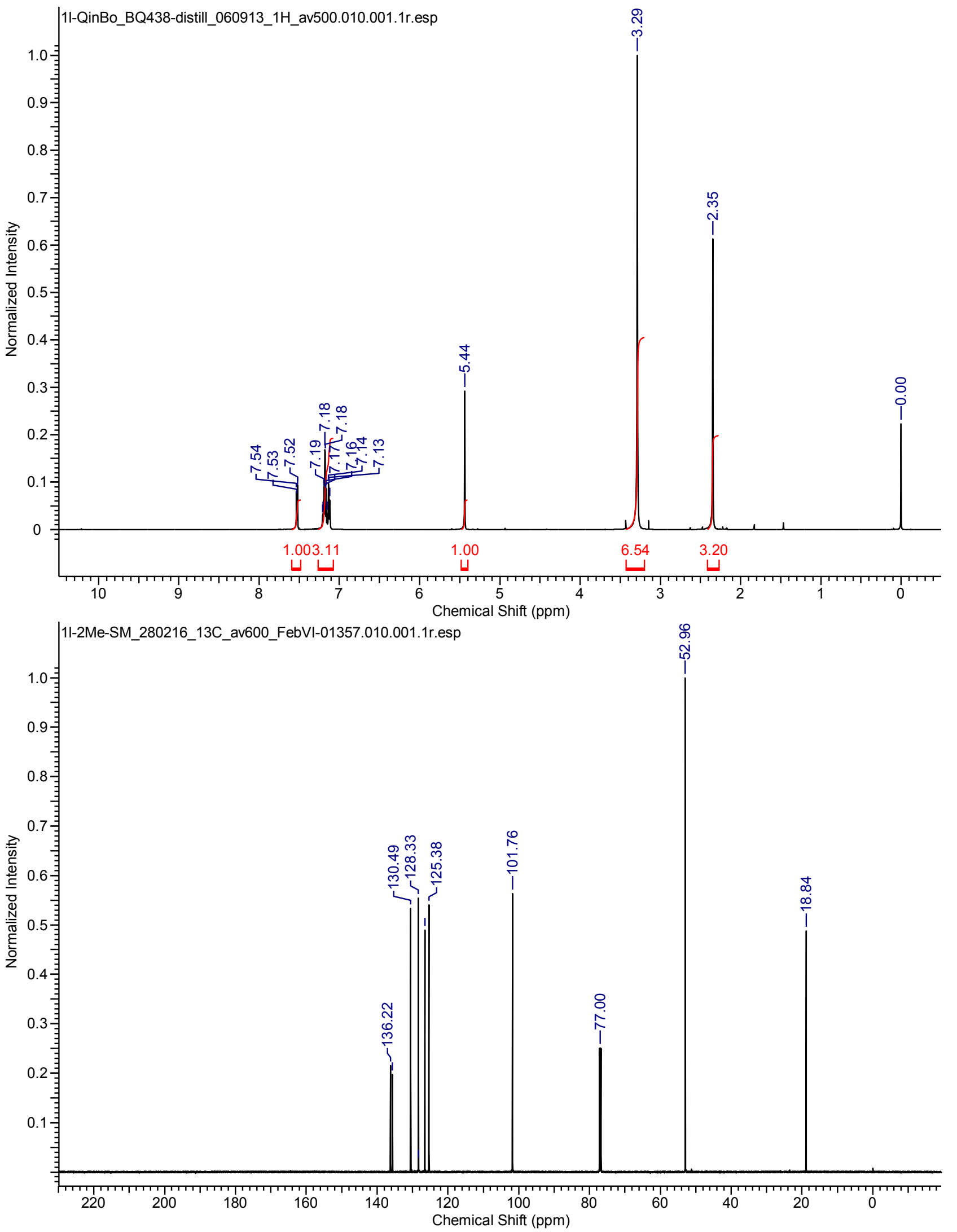


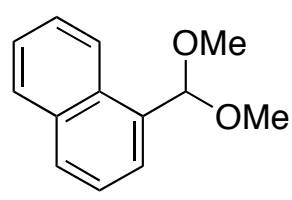

$1 \mathrm{~m}$
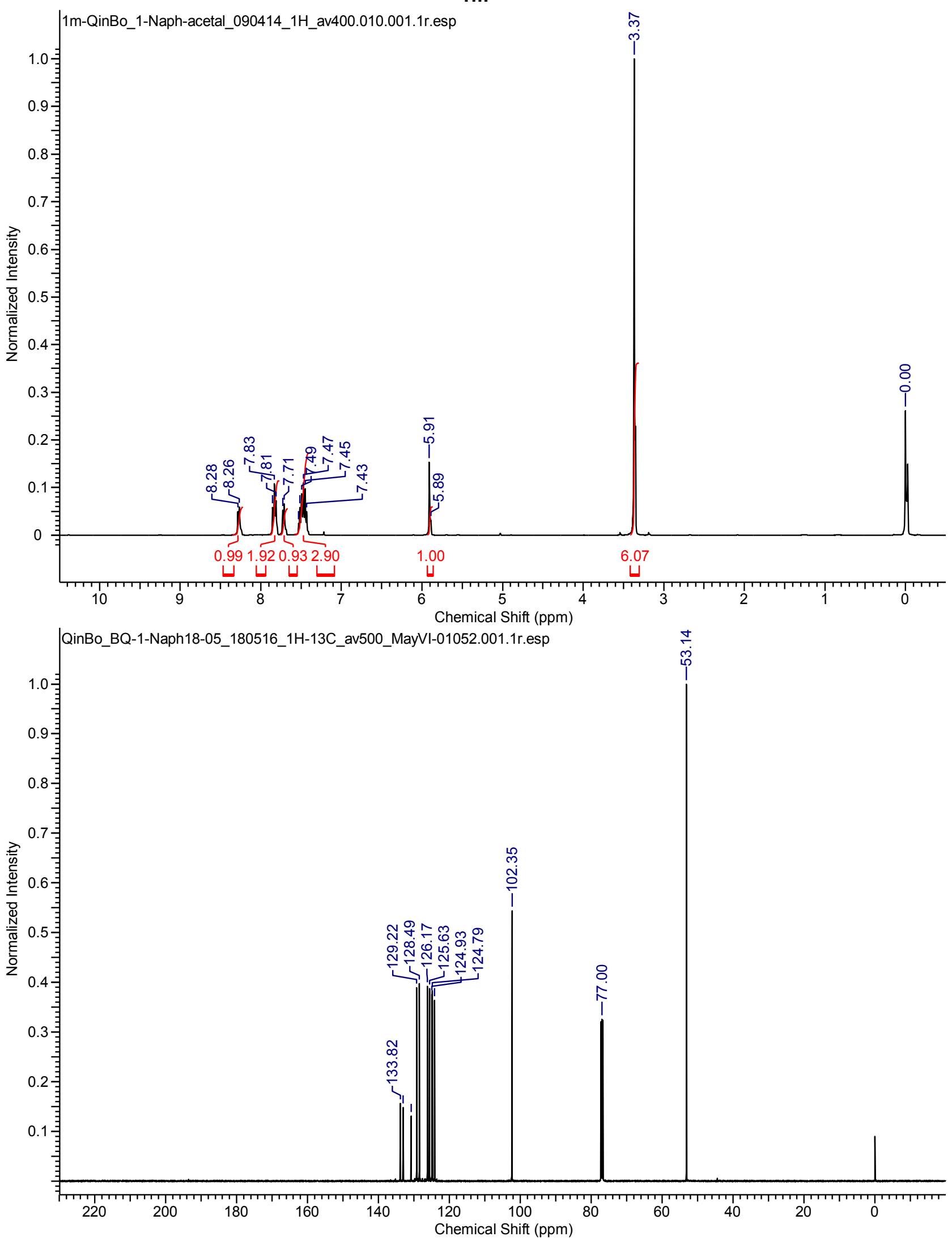

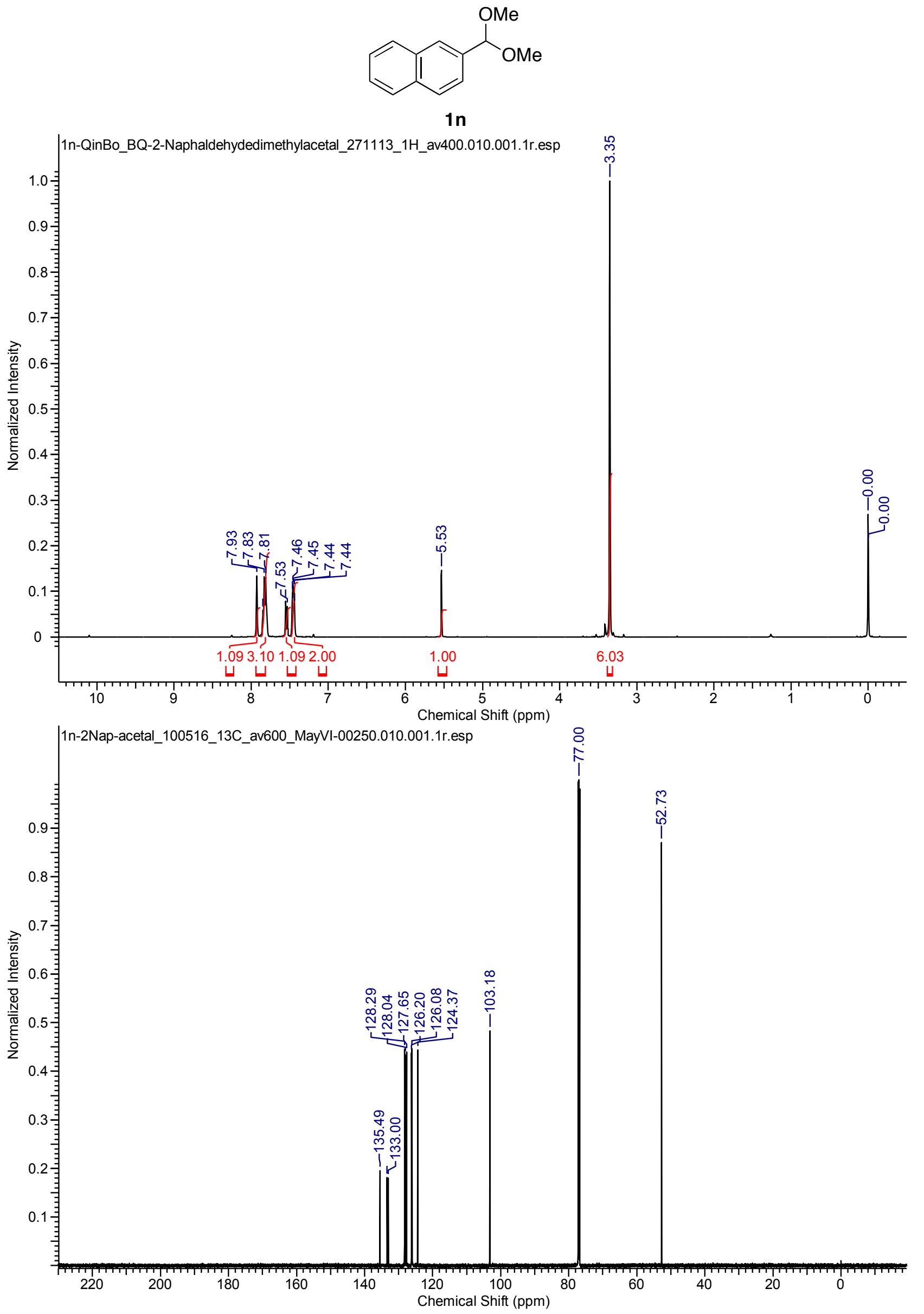


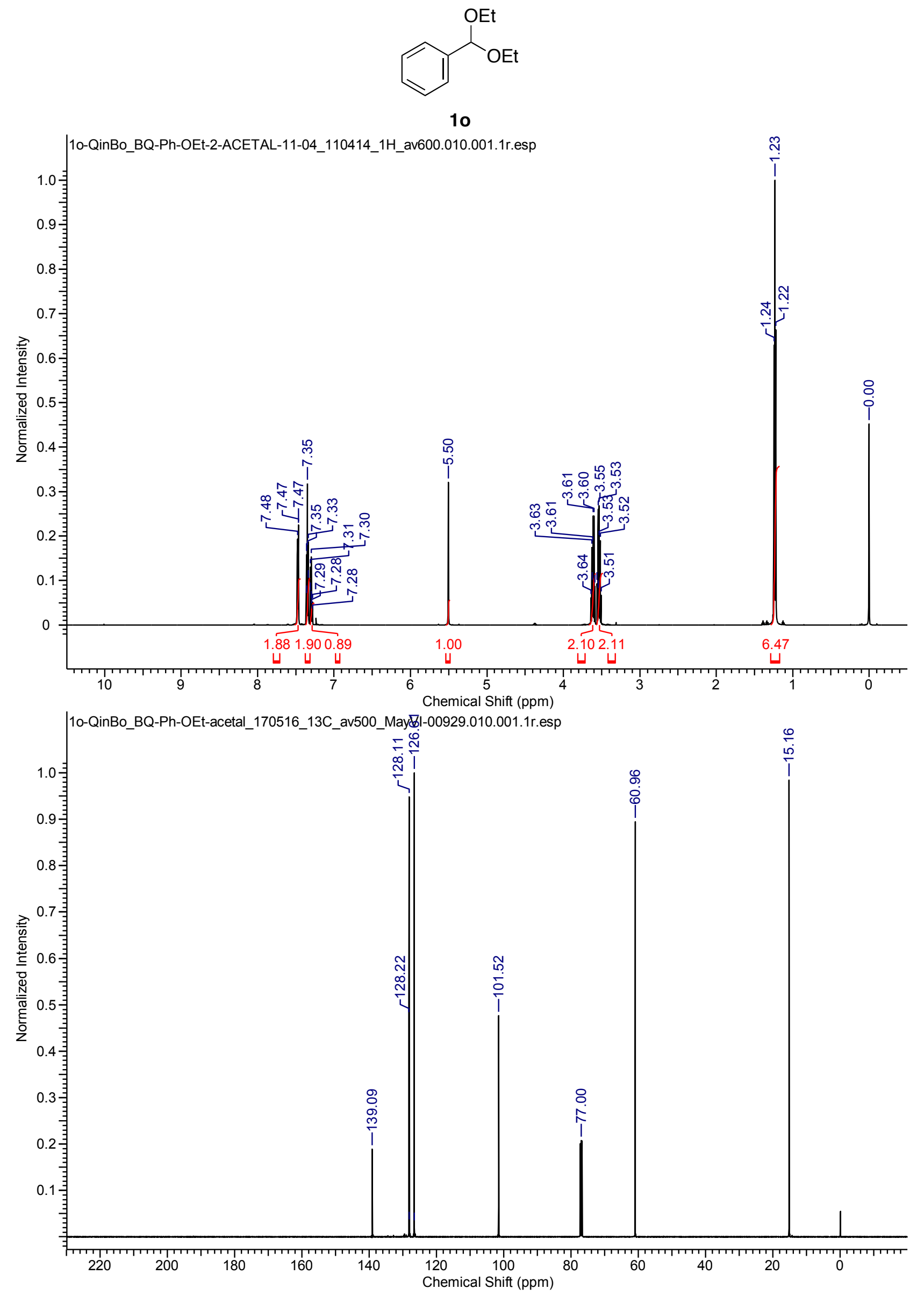




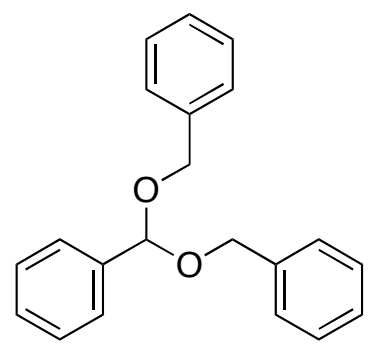

$1 p$
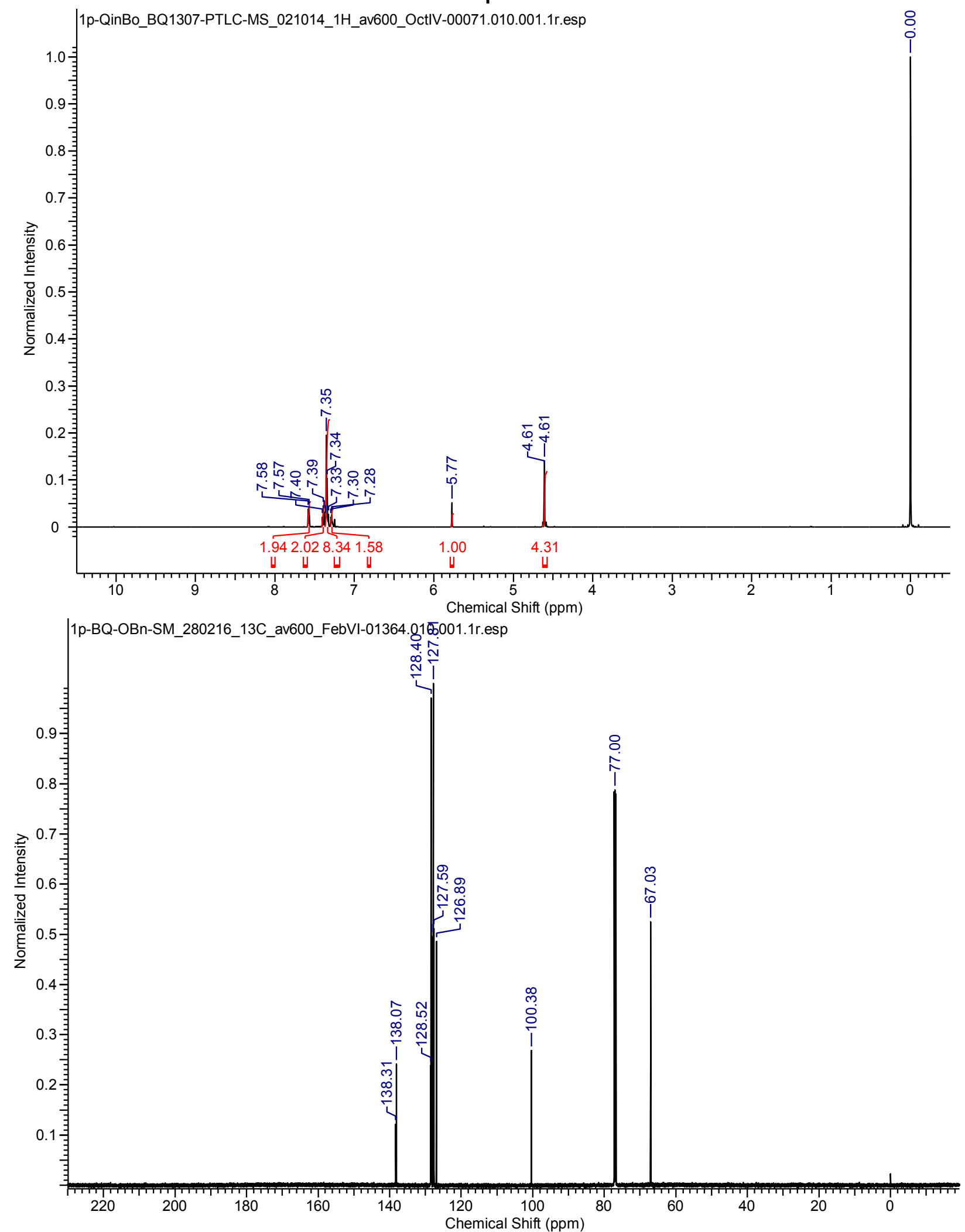


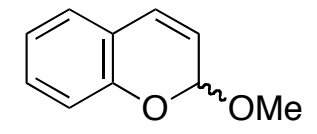

rac-1q
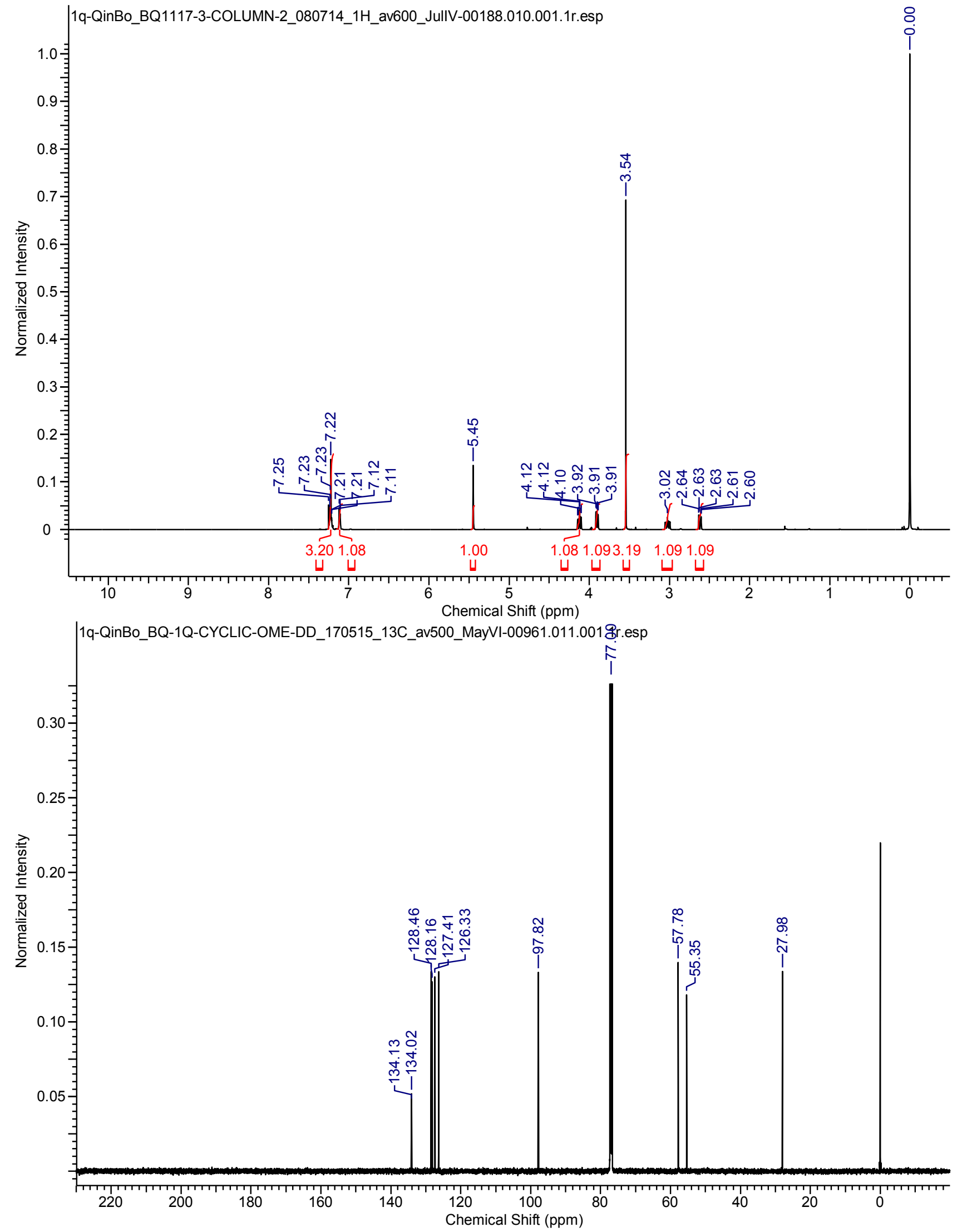


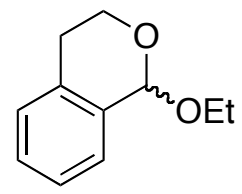

rac-1r
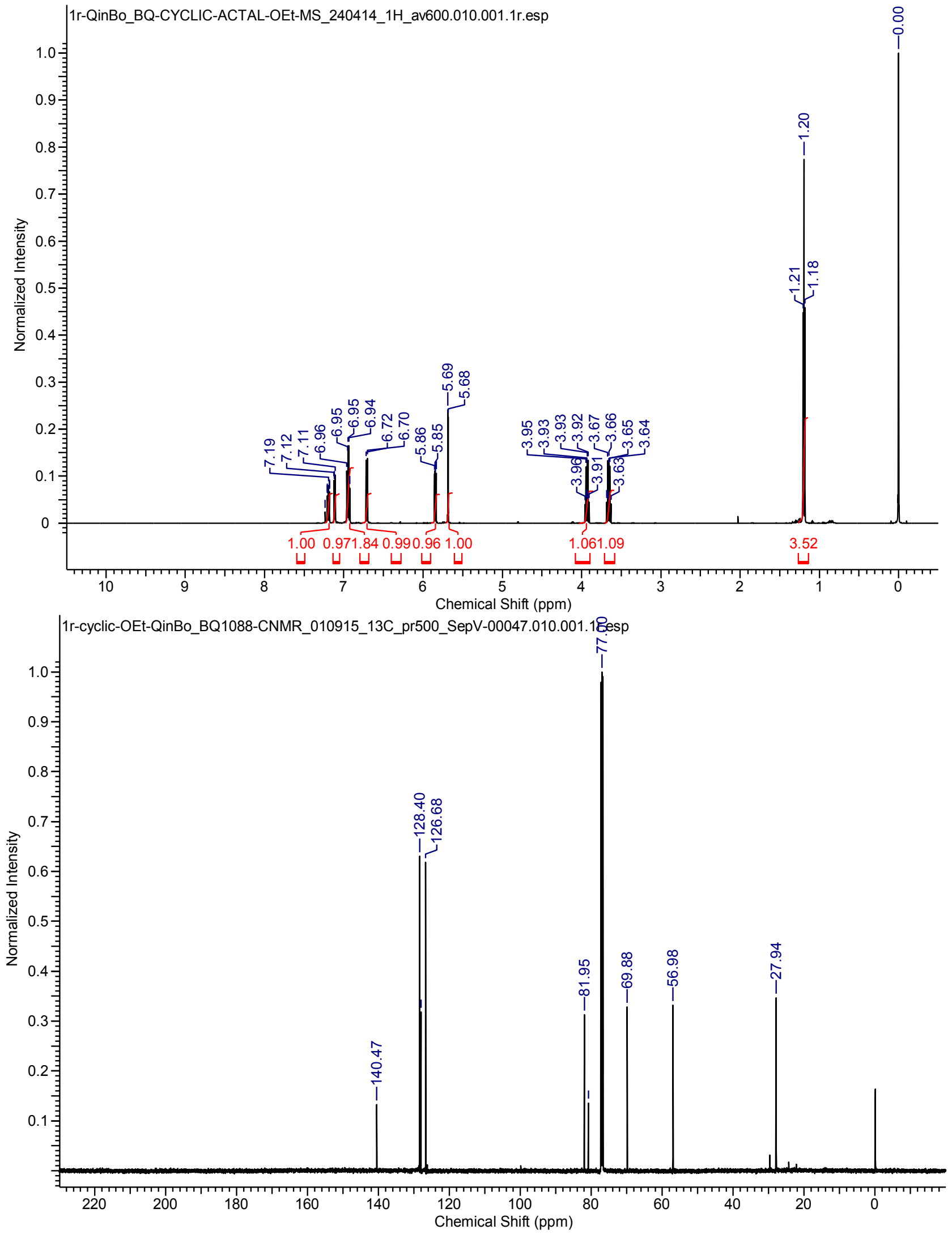


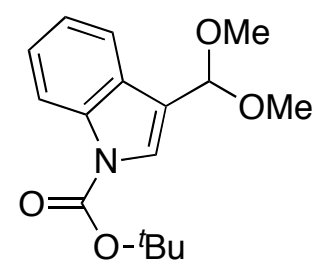

$1 \mathrm{~s}$
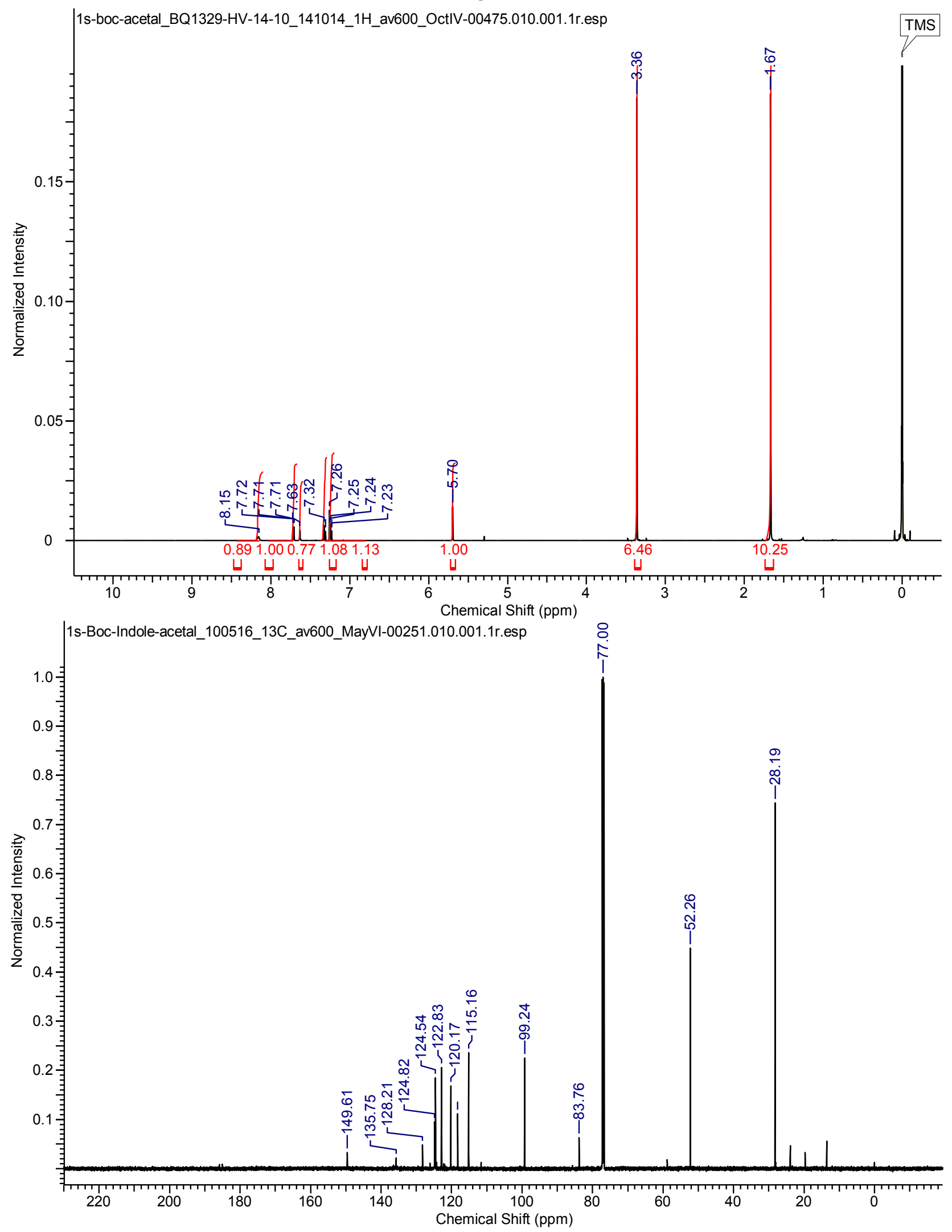


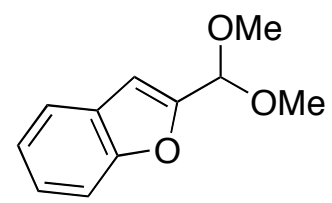

$1 t$
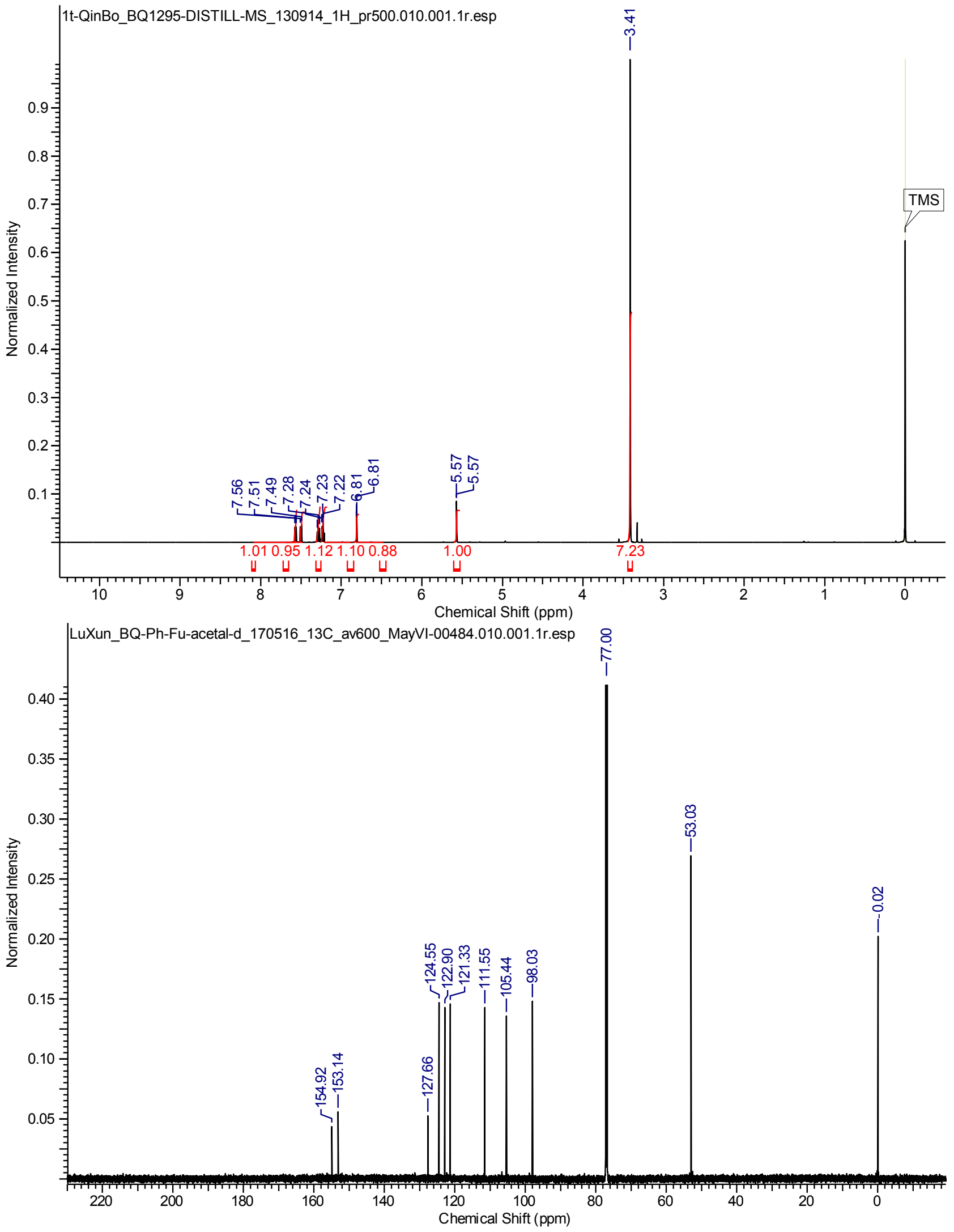


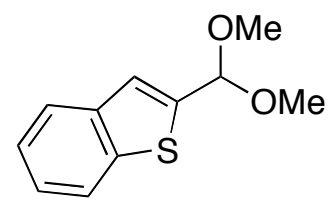

$1 \mathrm{u}$
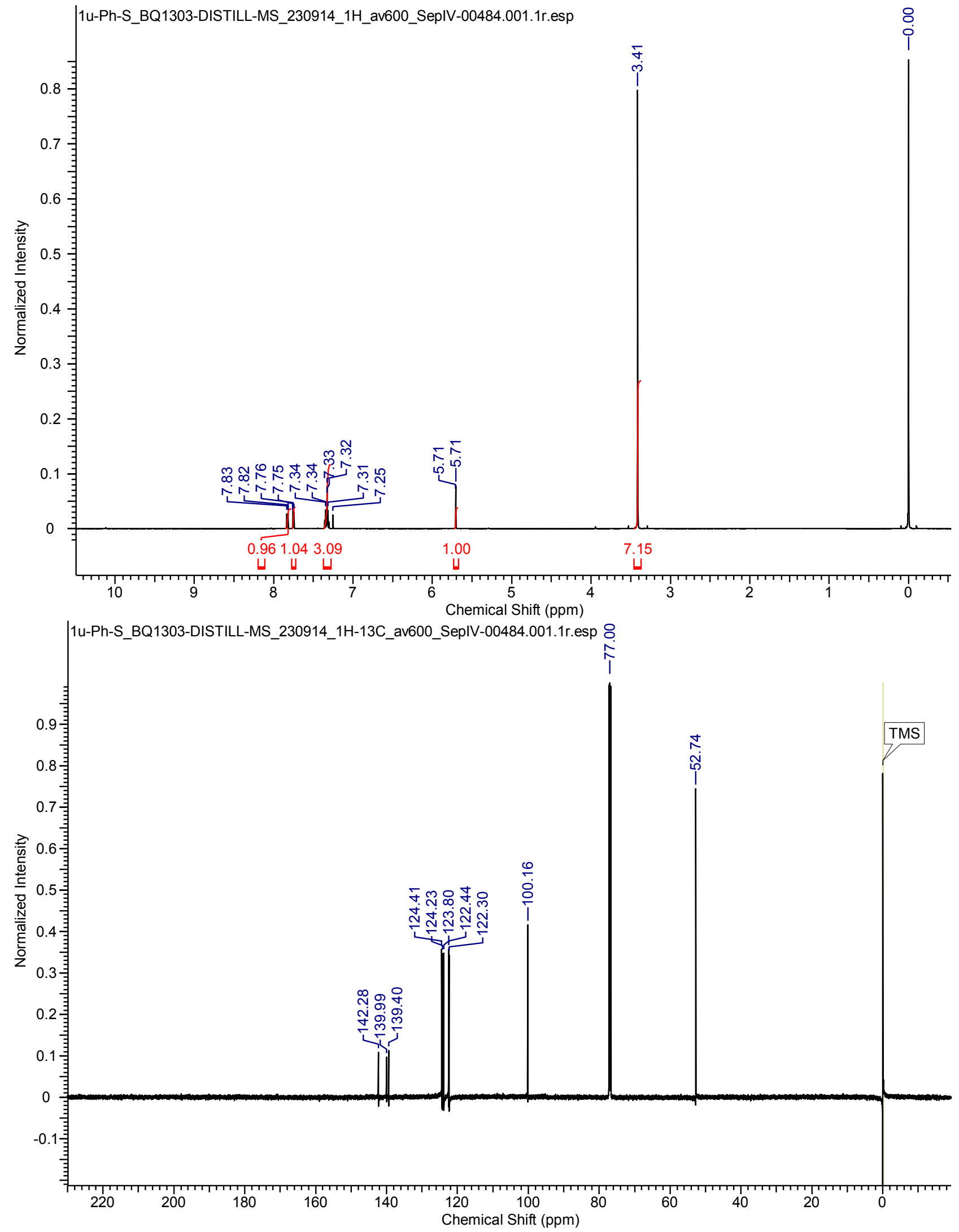


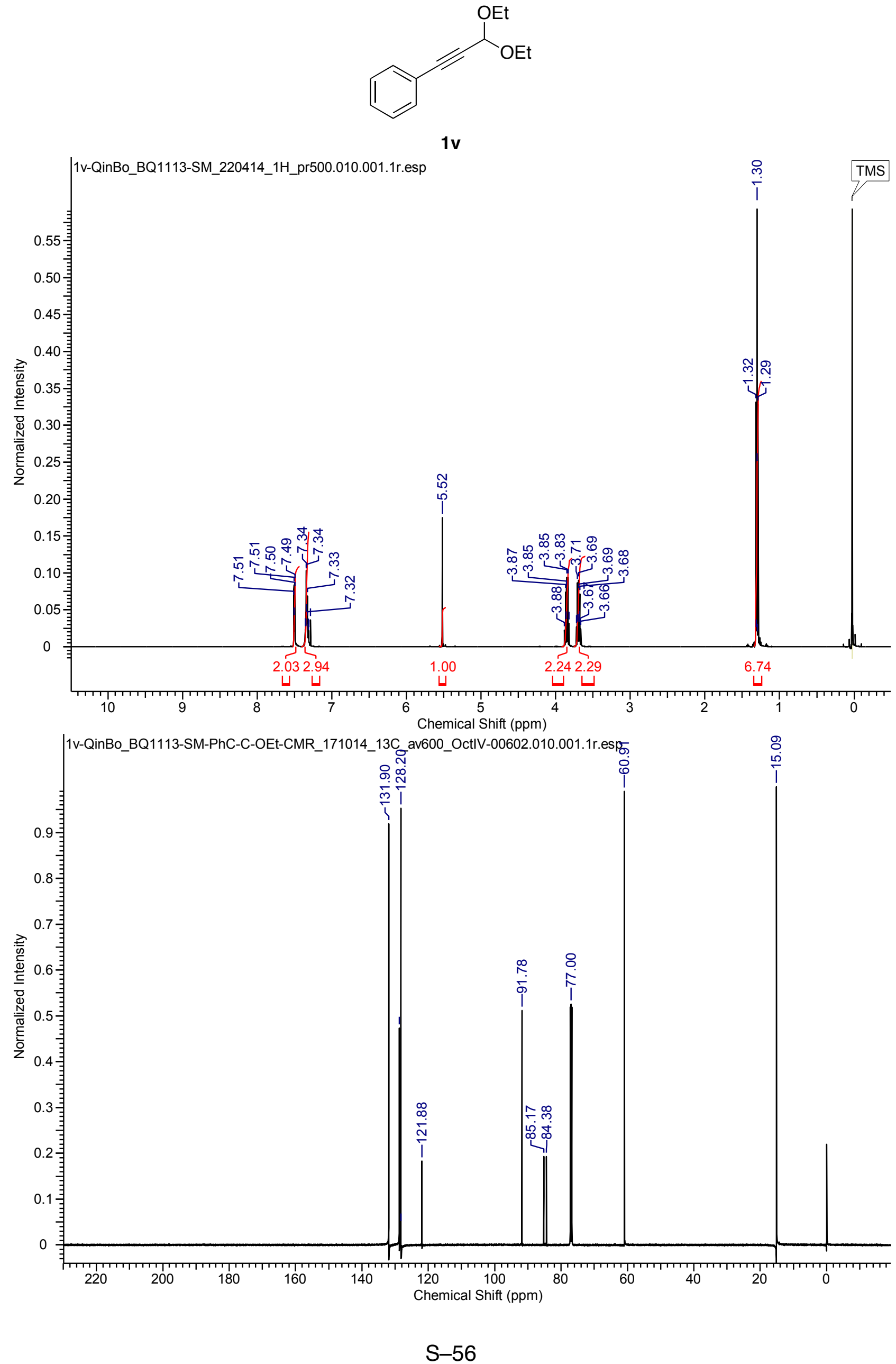




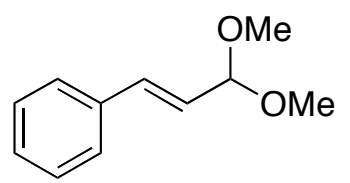

$1 \mathrm{w}$
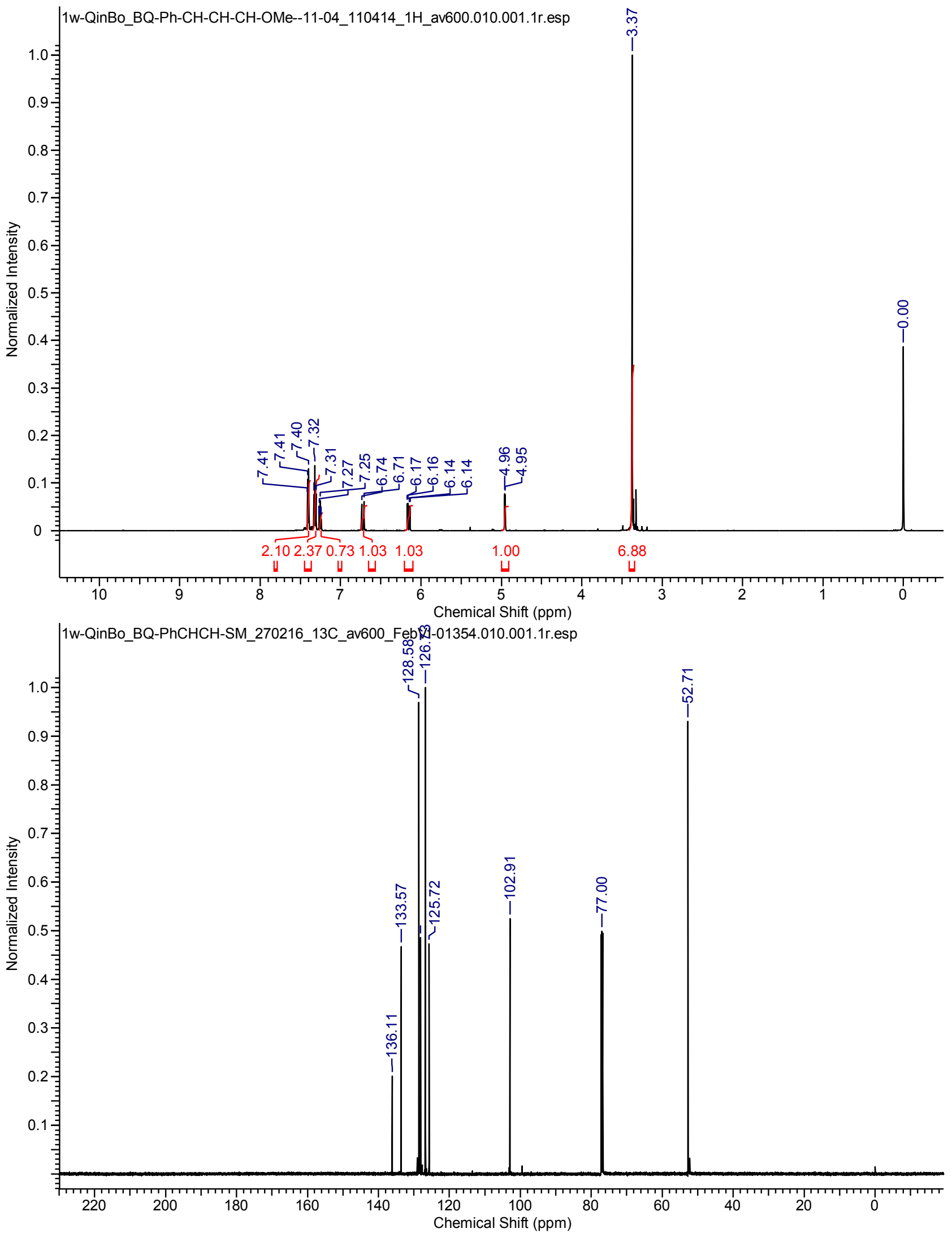


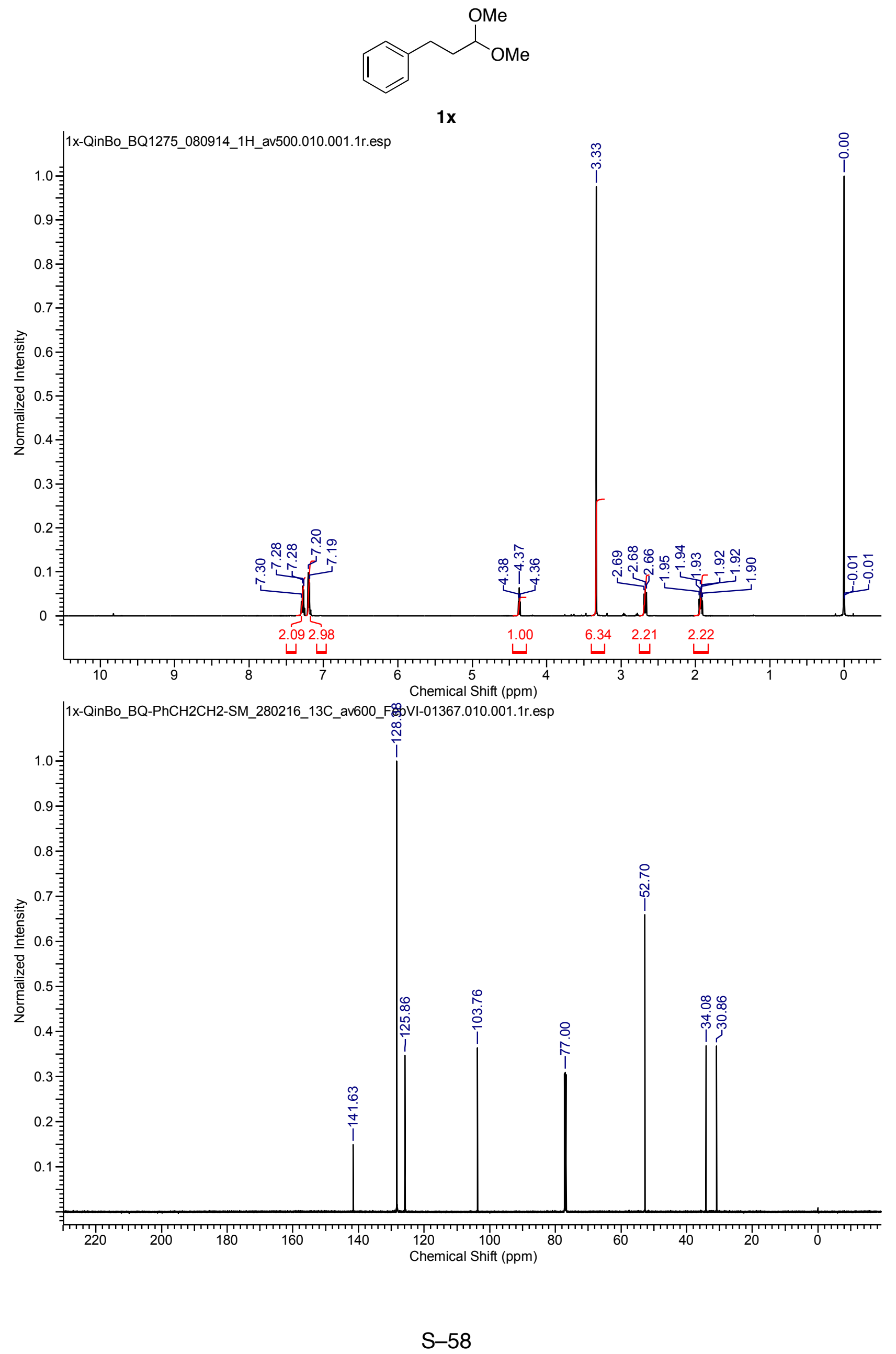




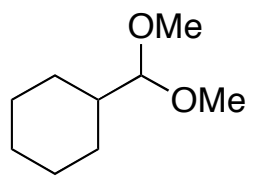

$1 y$
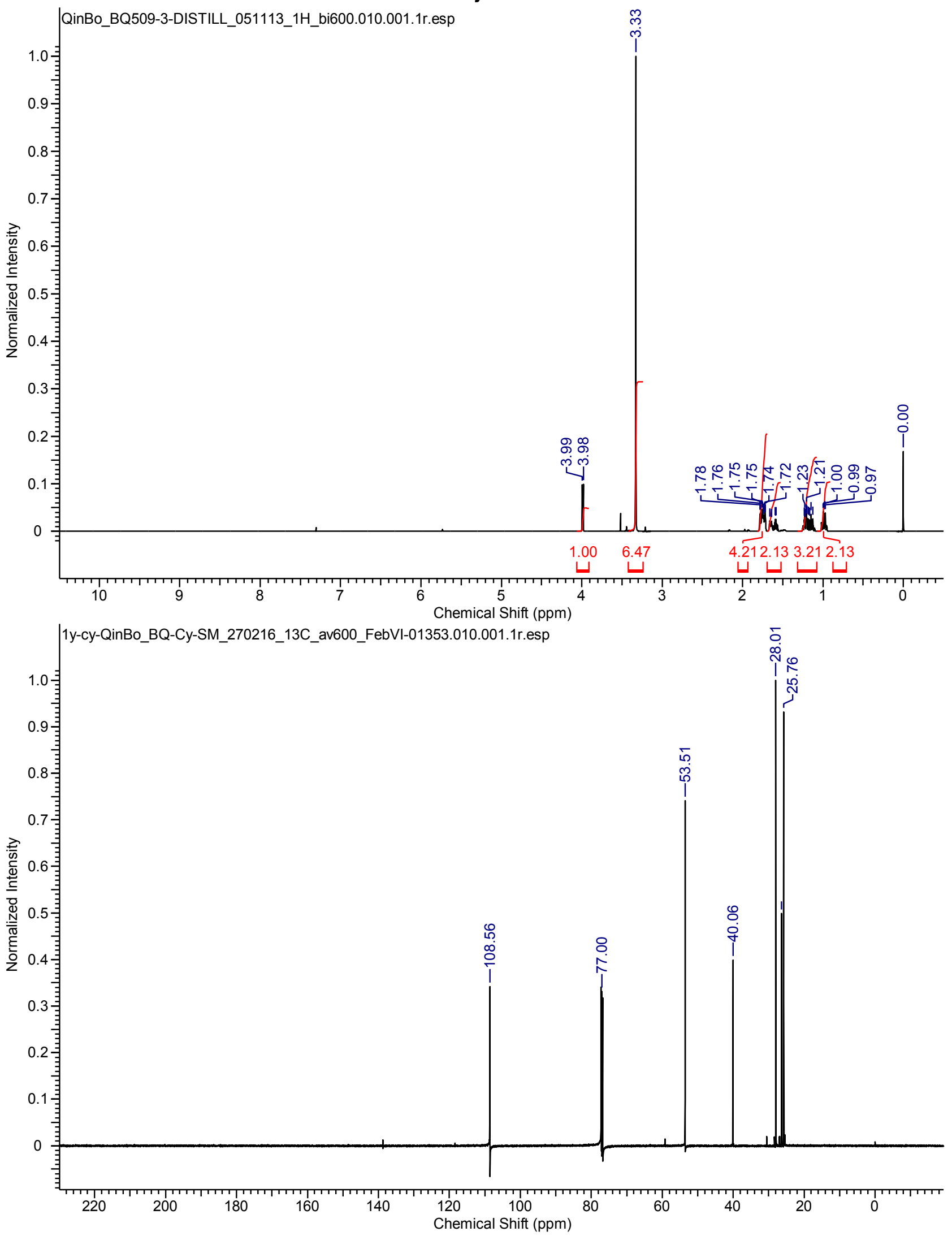


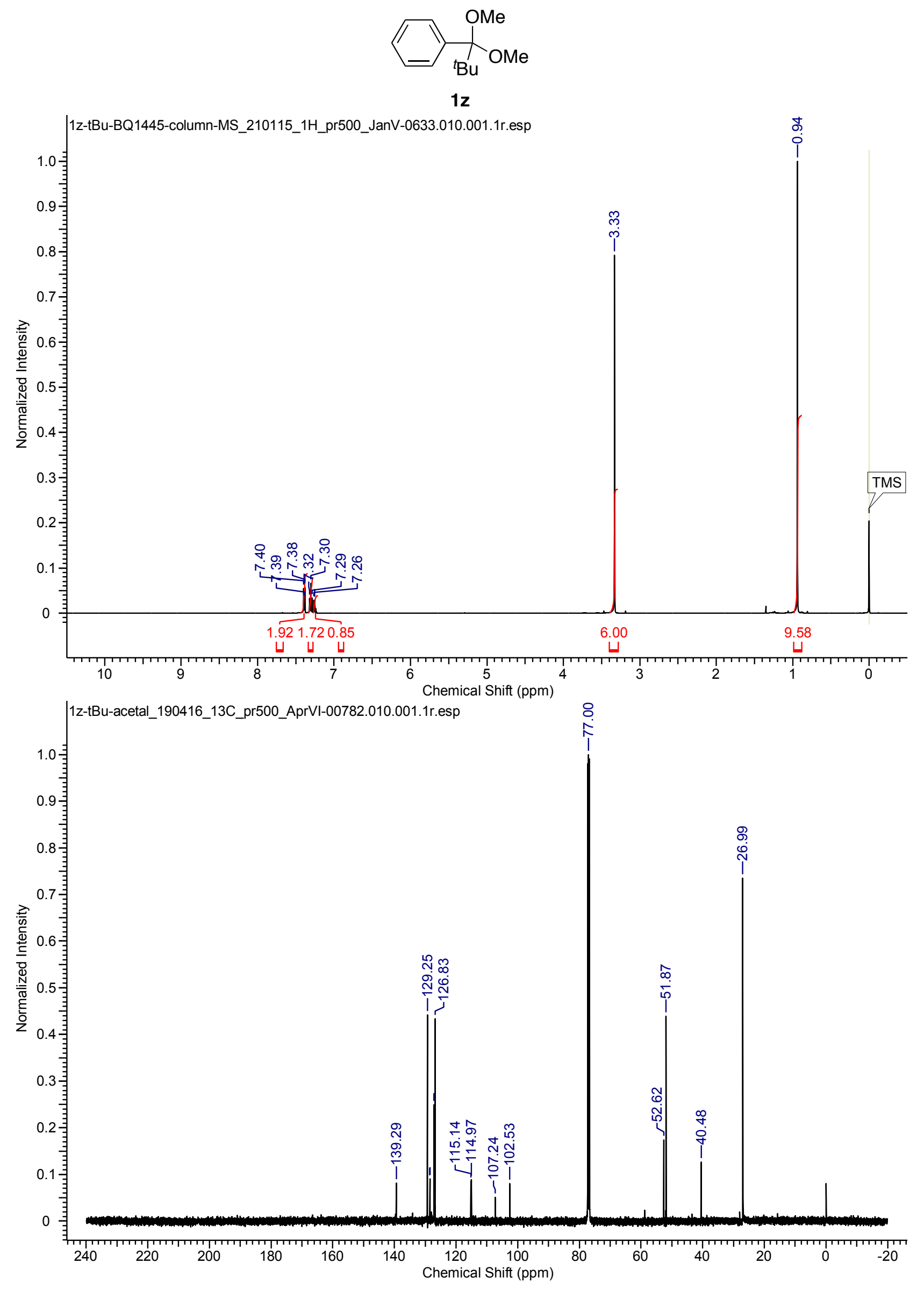




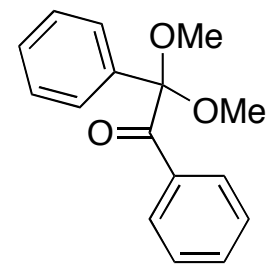

$1 z^{\prime}$
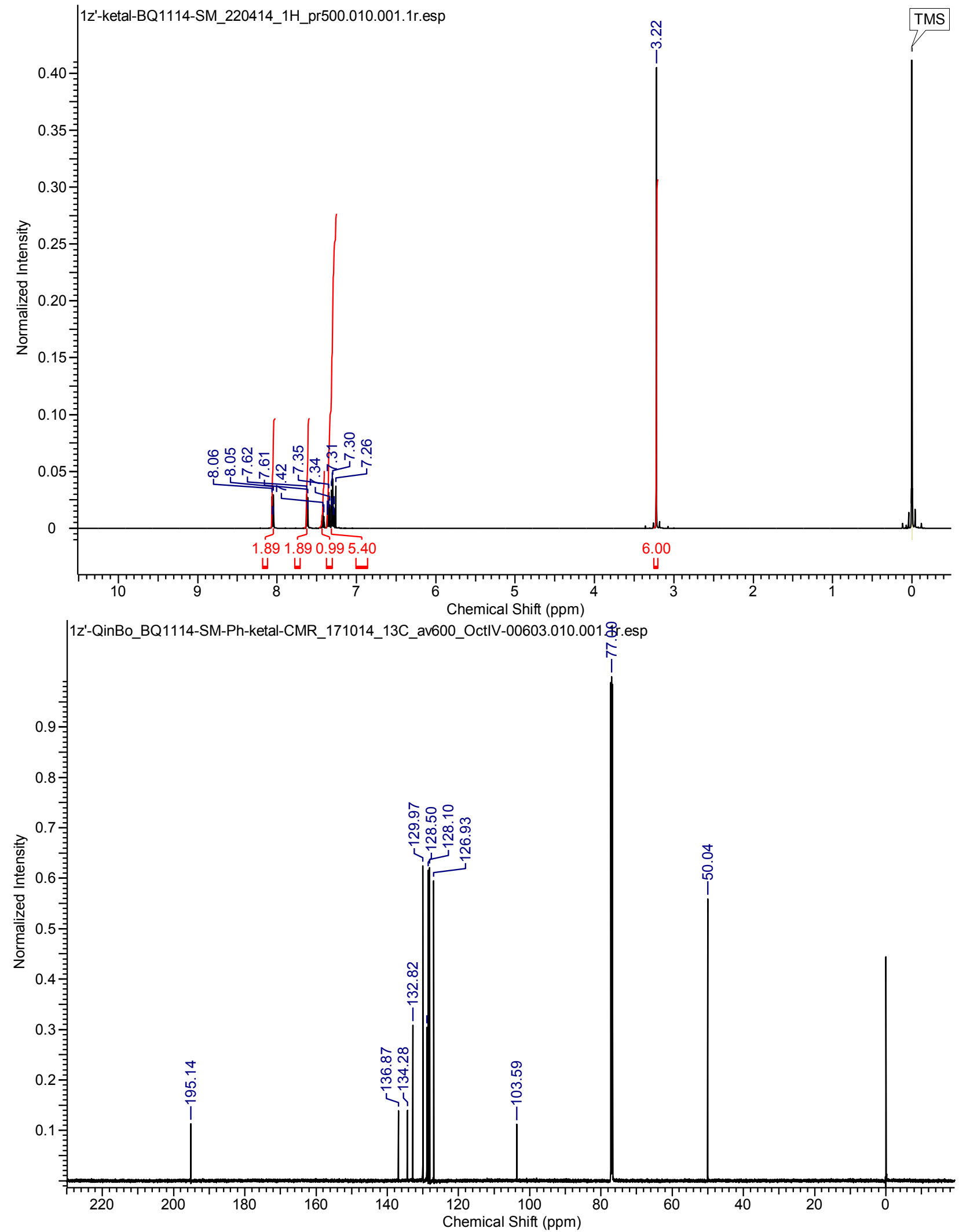


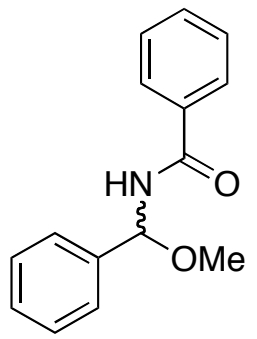

rac-12
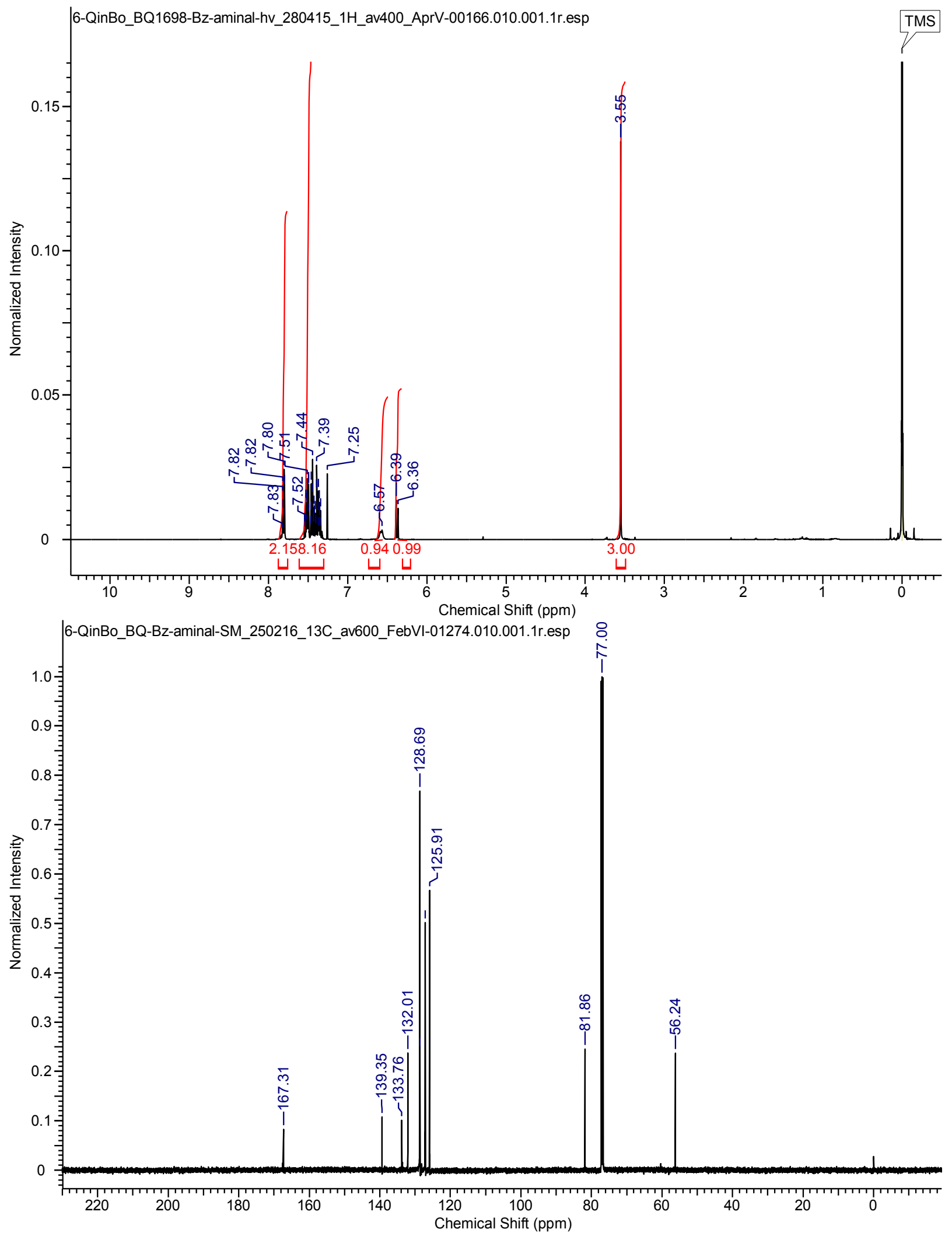


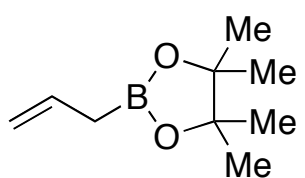

2
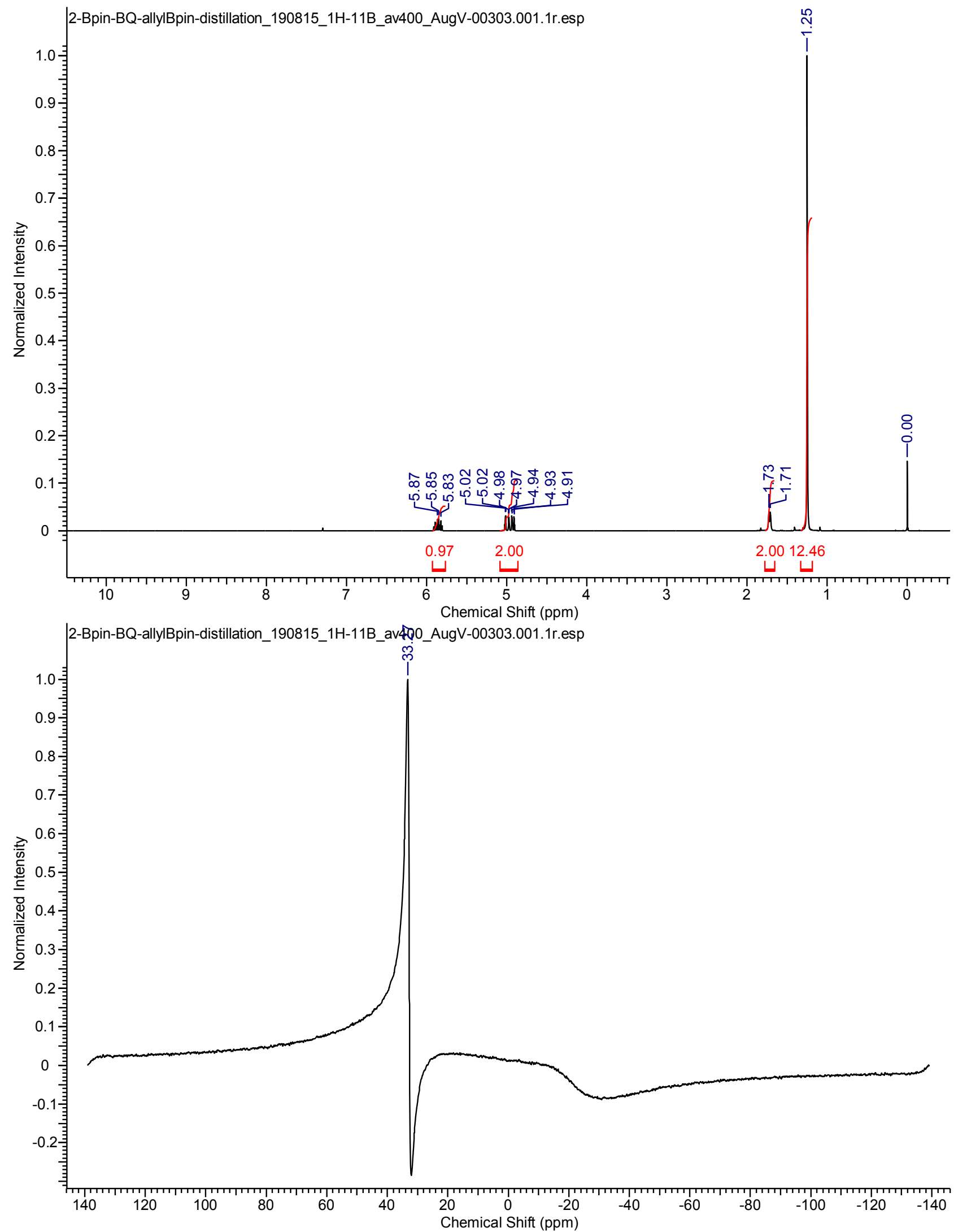


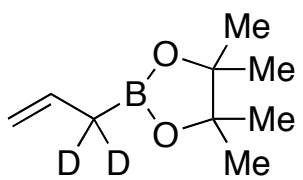

\section{2- $\left[d_{2}\right]$}
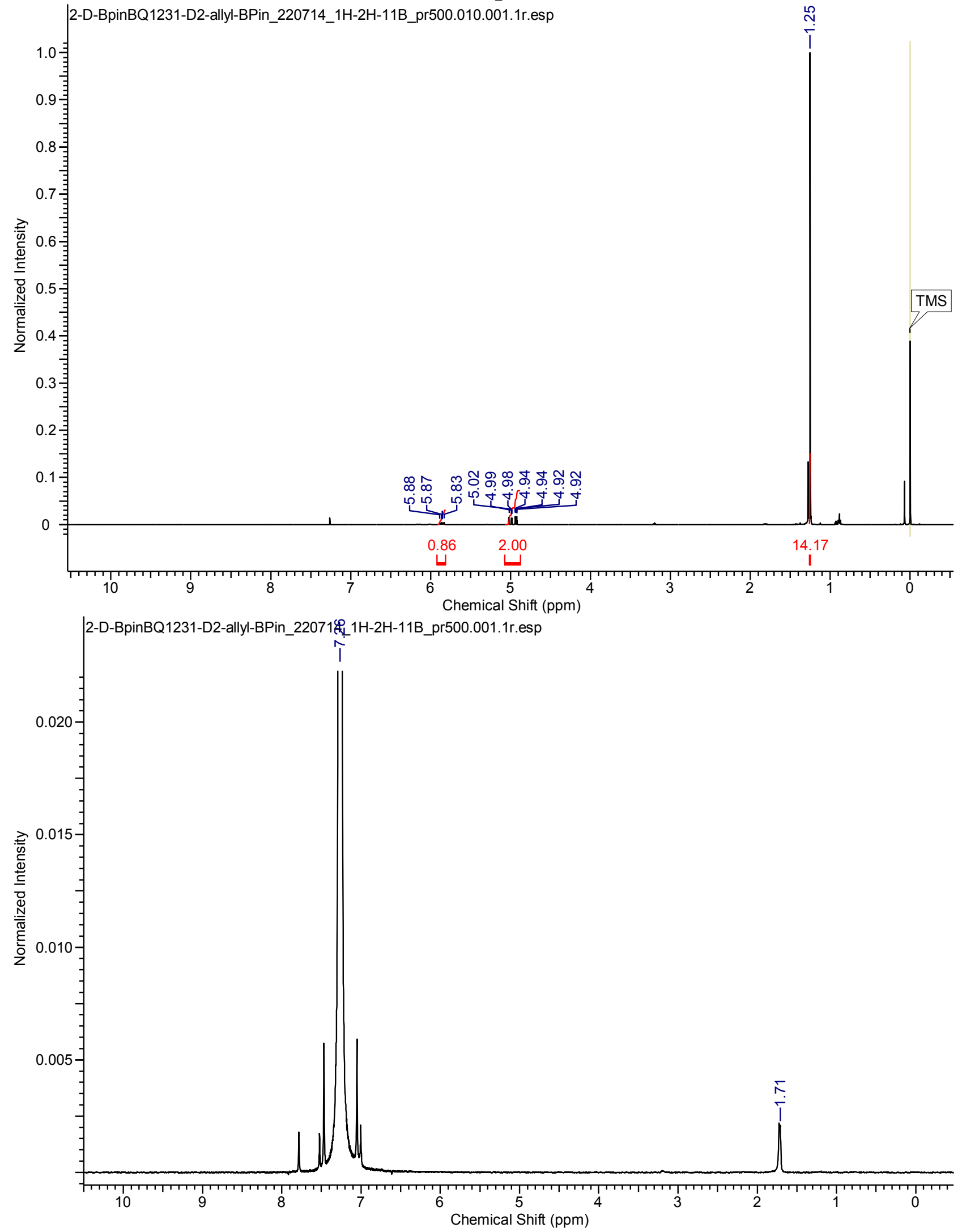


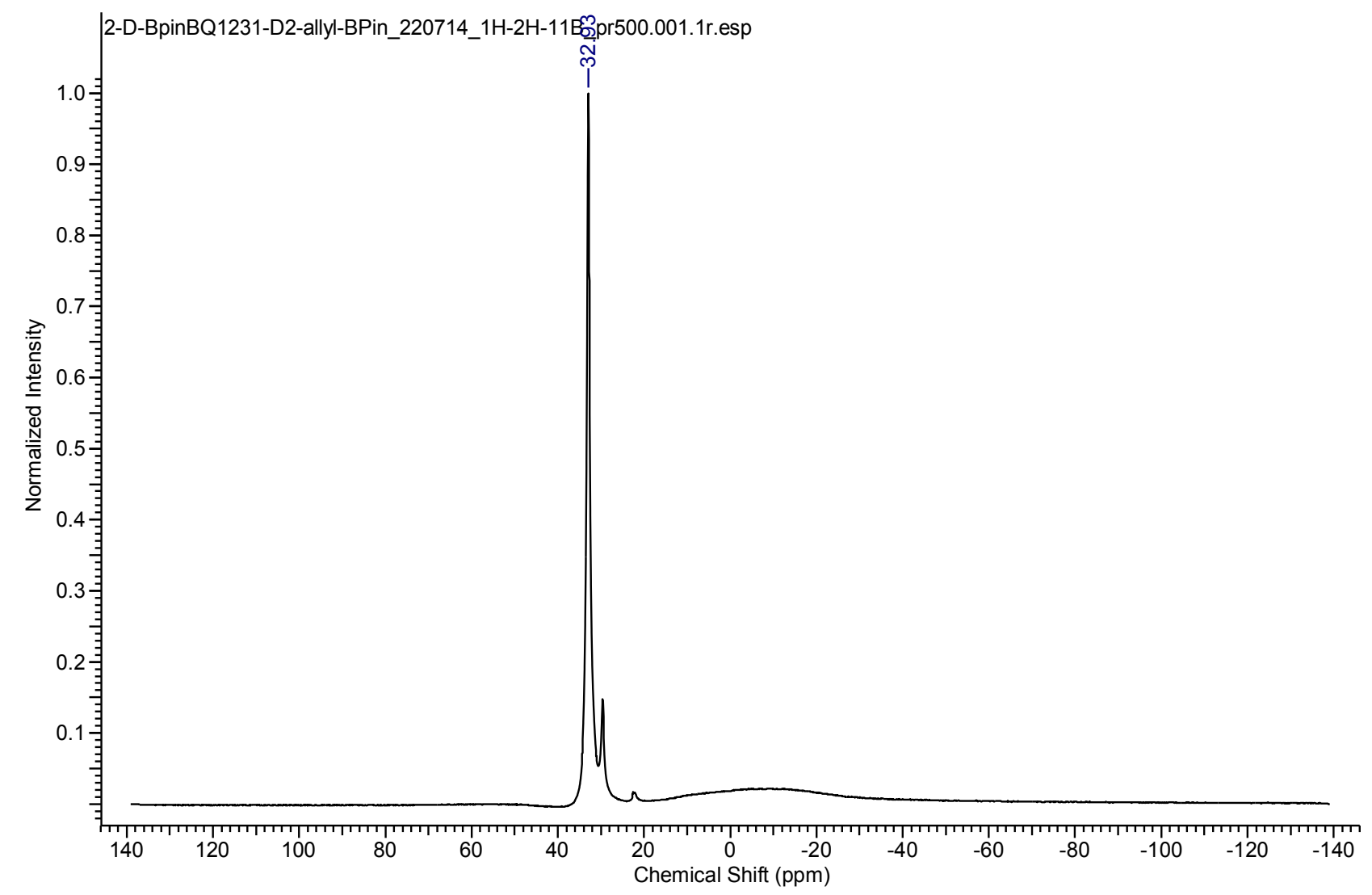




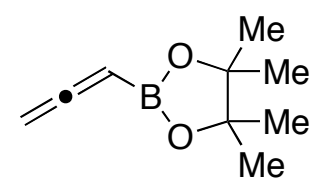

14
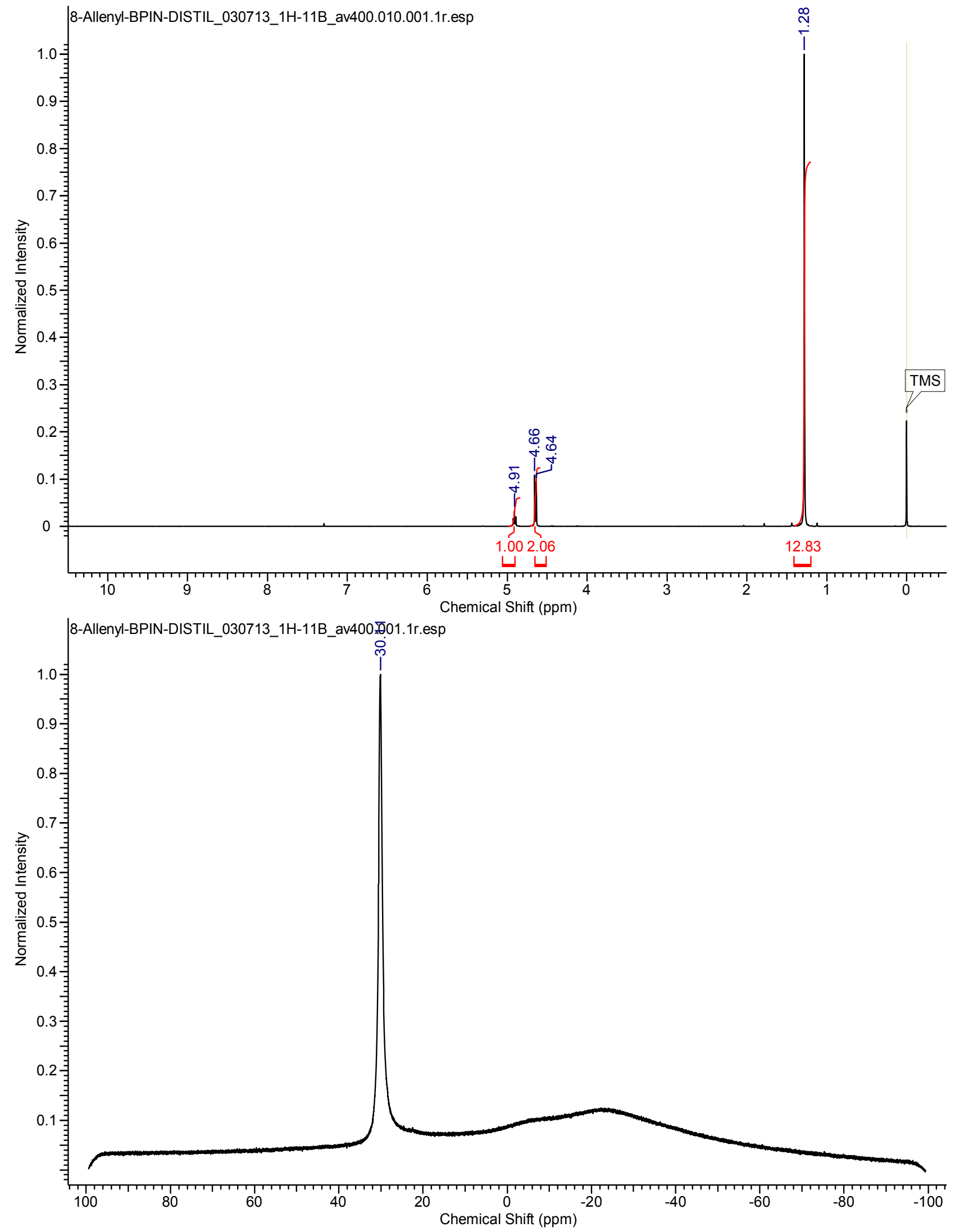


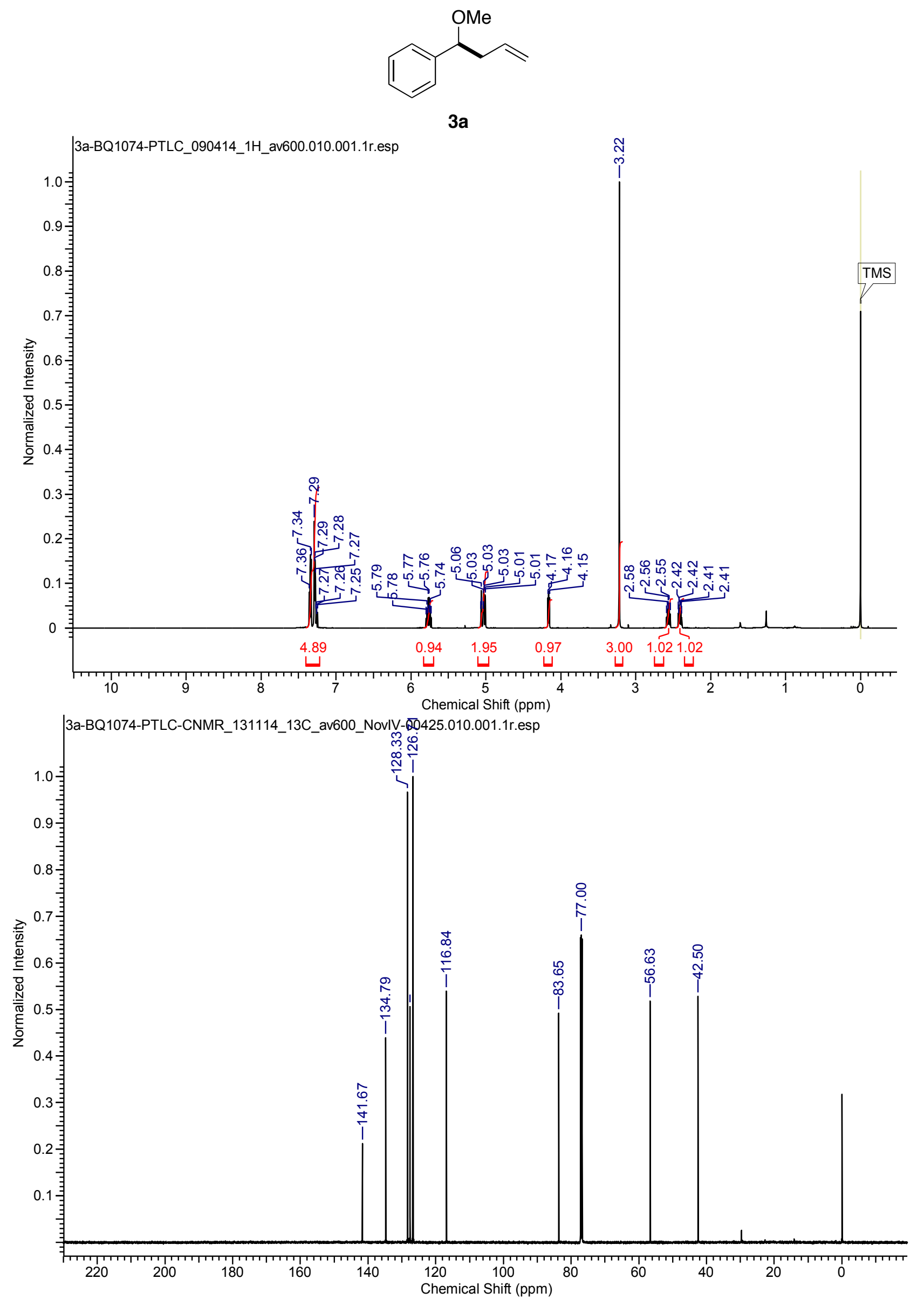


<smiles>C=CCC(OC)c1ccc(C(F)(F)F)cc1</smiles>

$3 b$
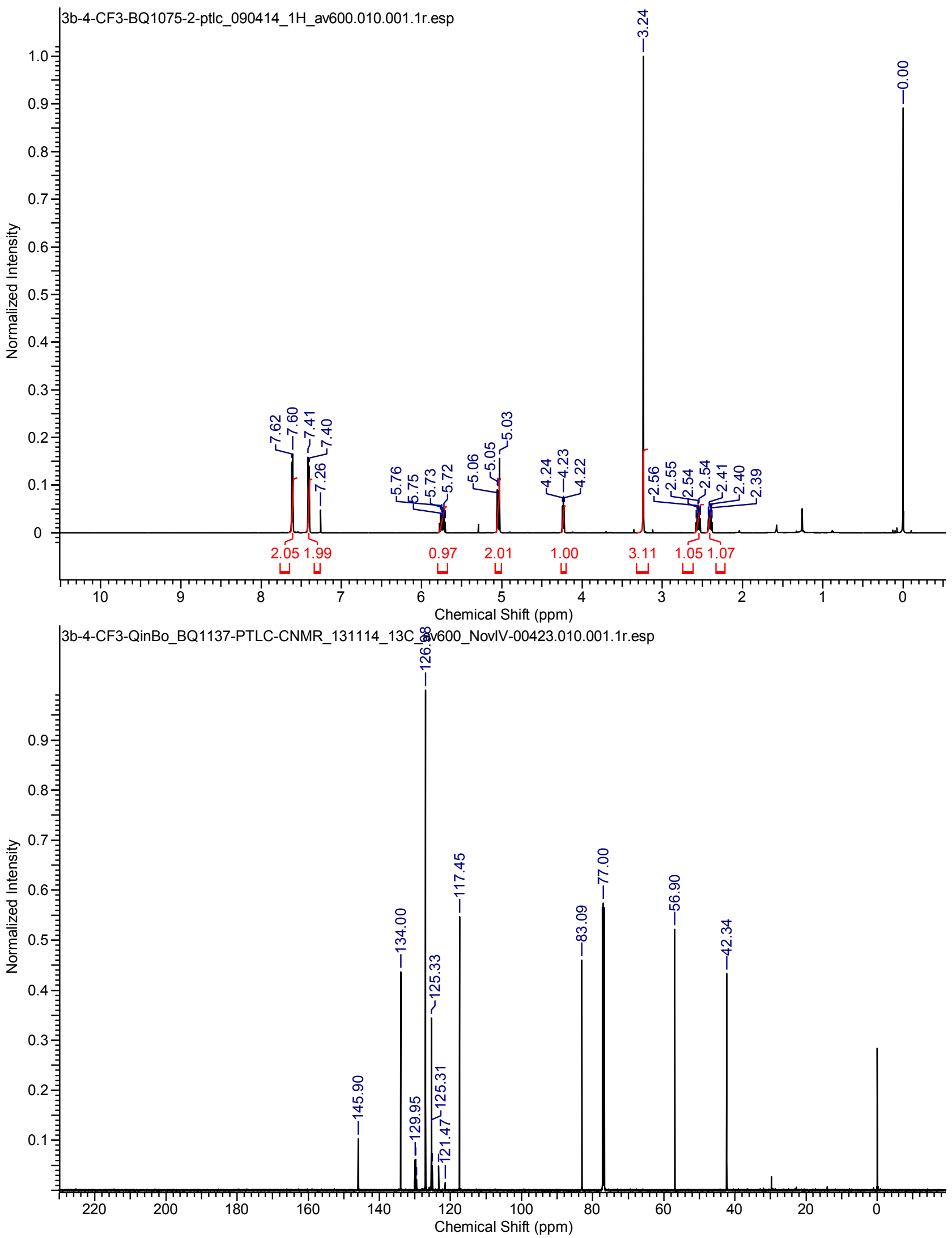


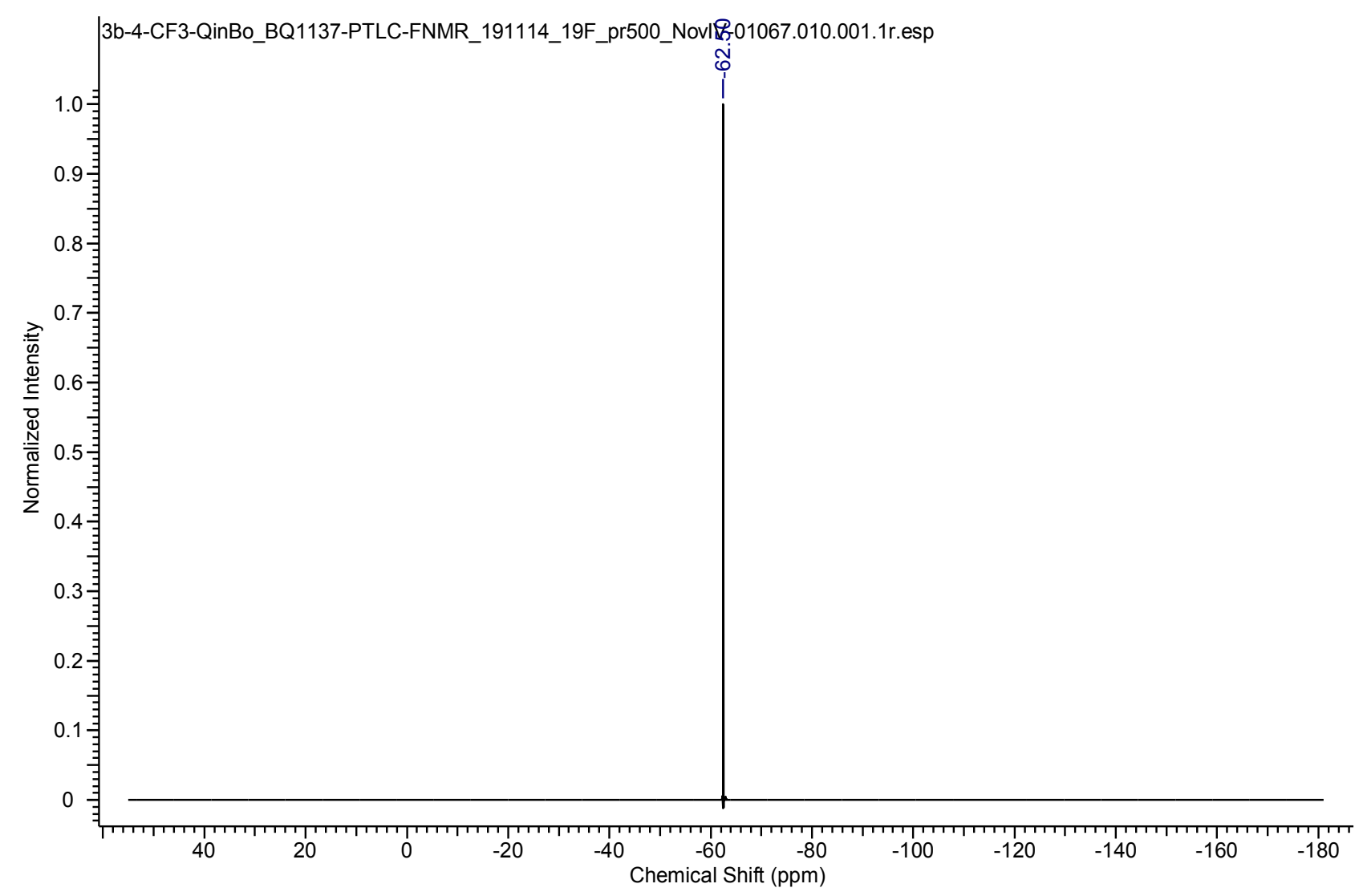


<smiles>C=CCC(OC)c1ccc(C(=O)OC)cc1</smiles>

3c
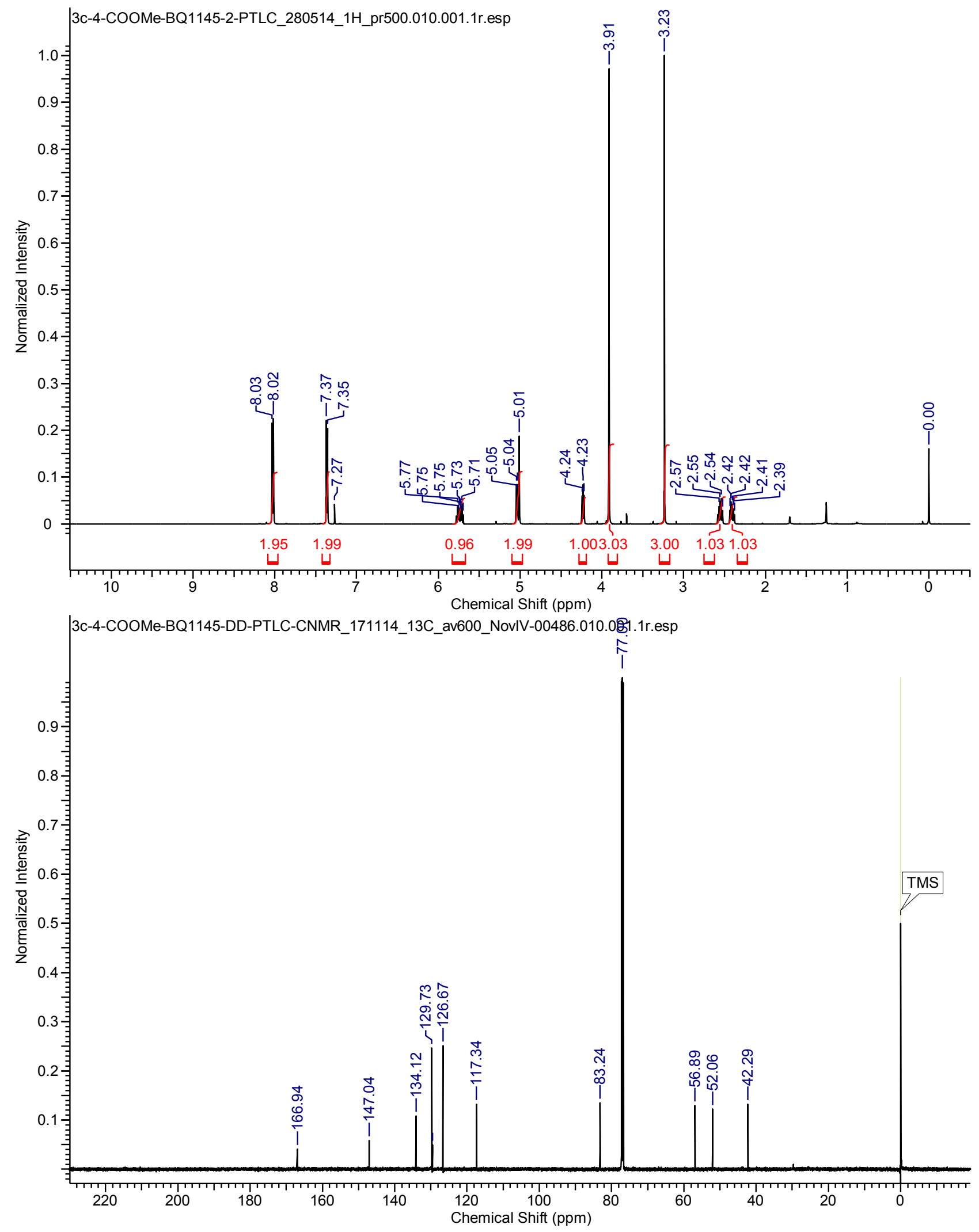


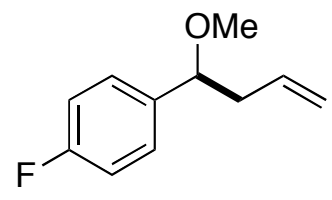

3d
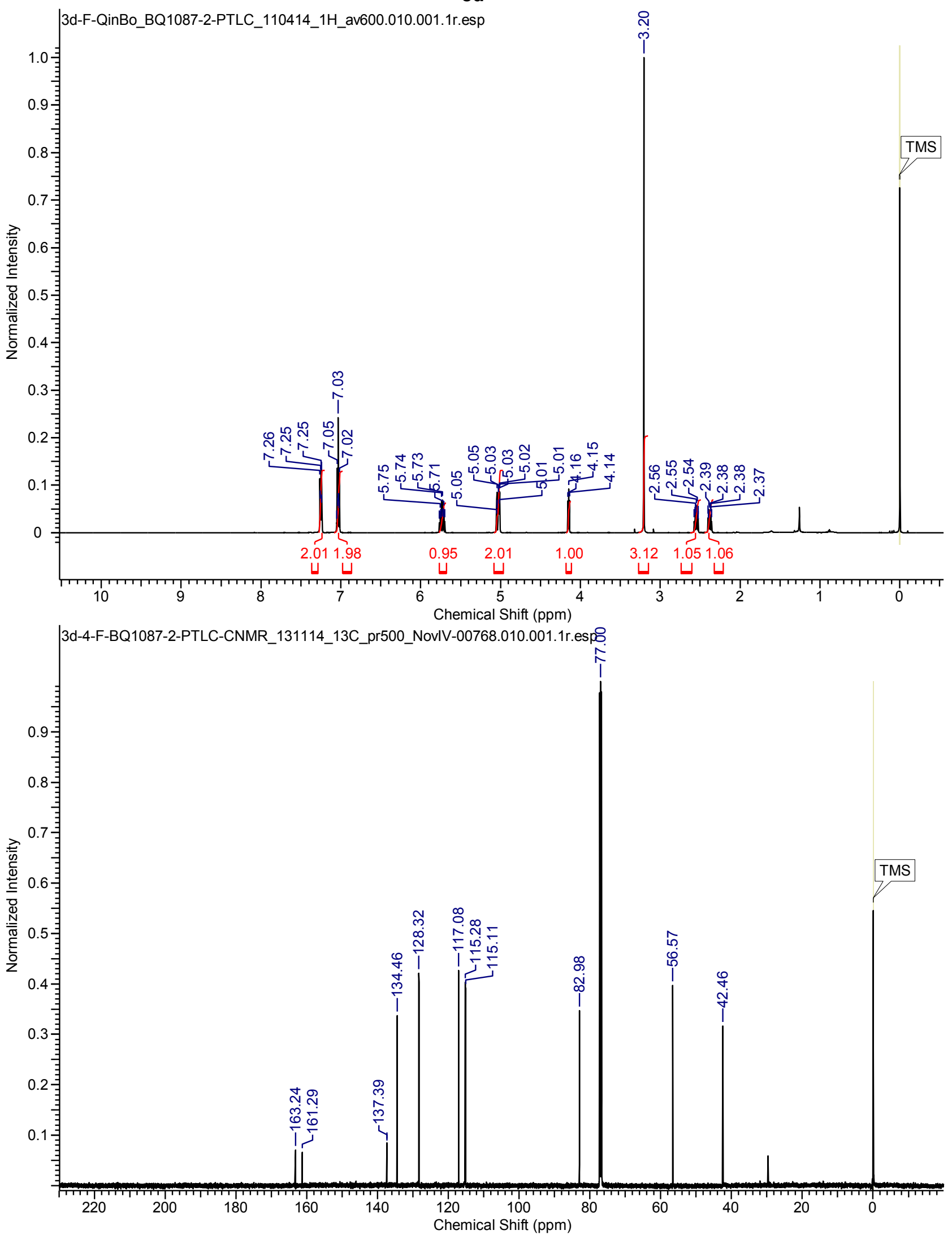


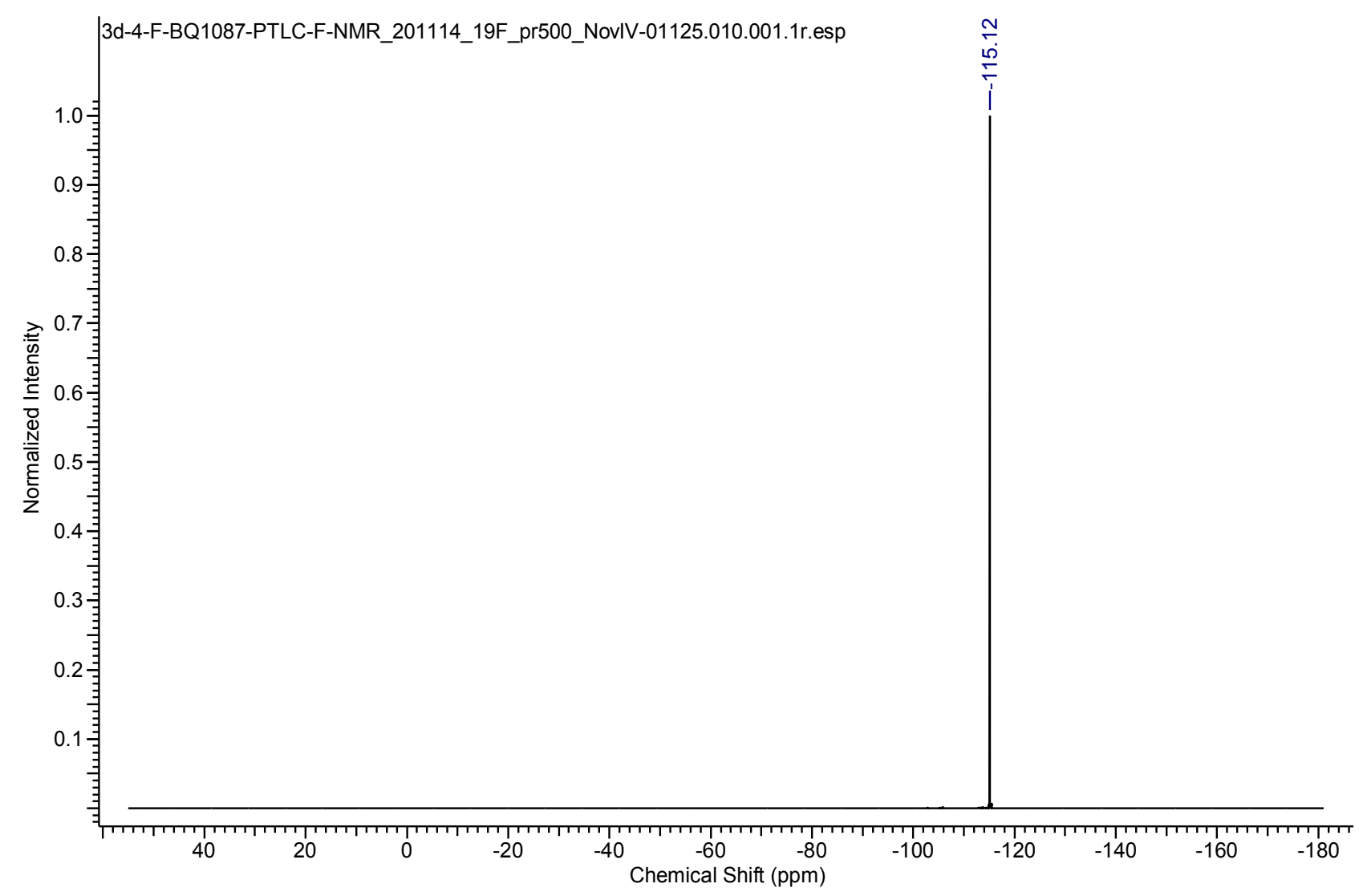



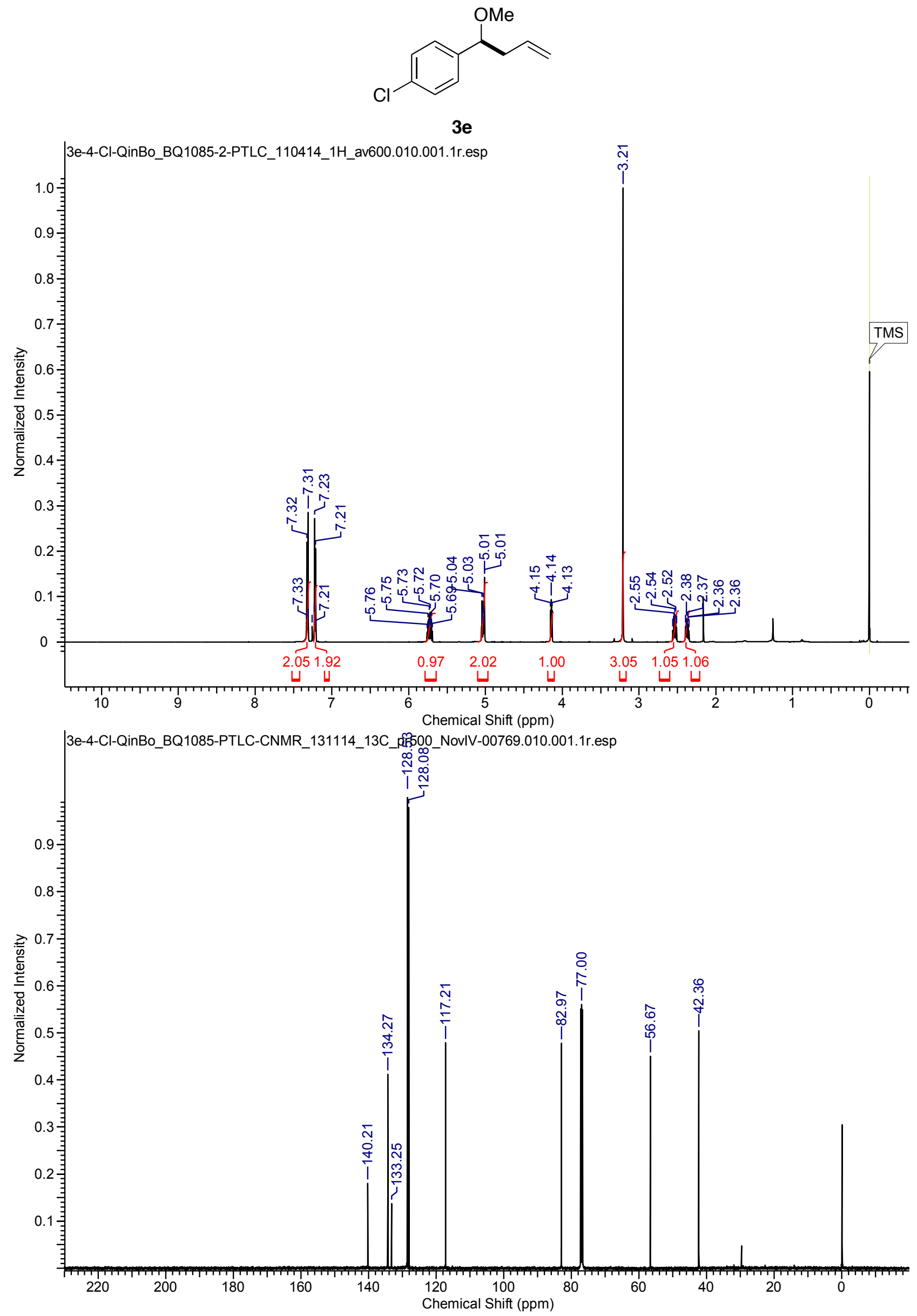

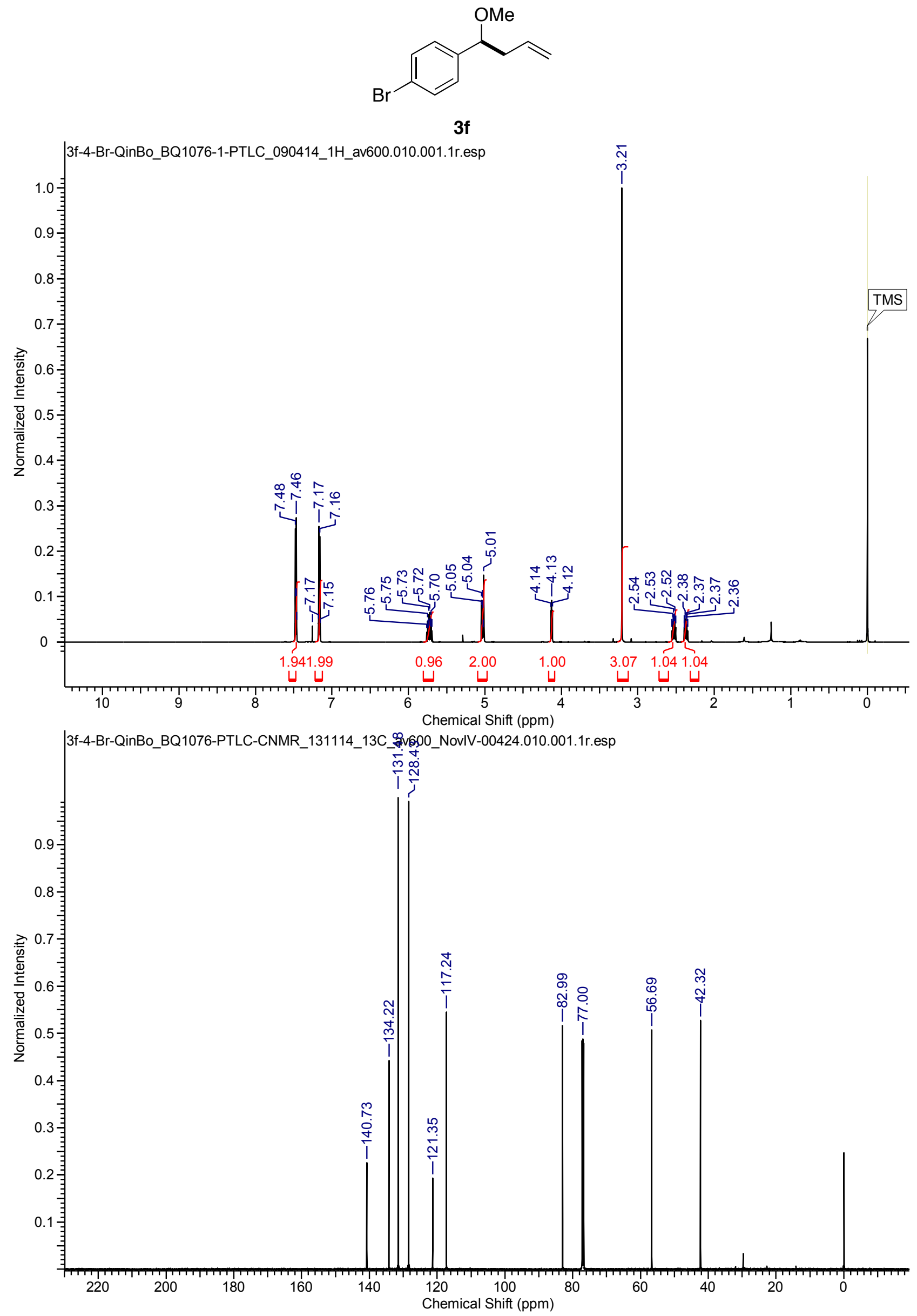

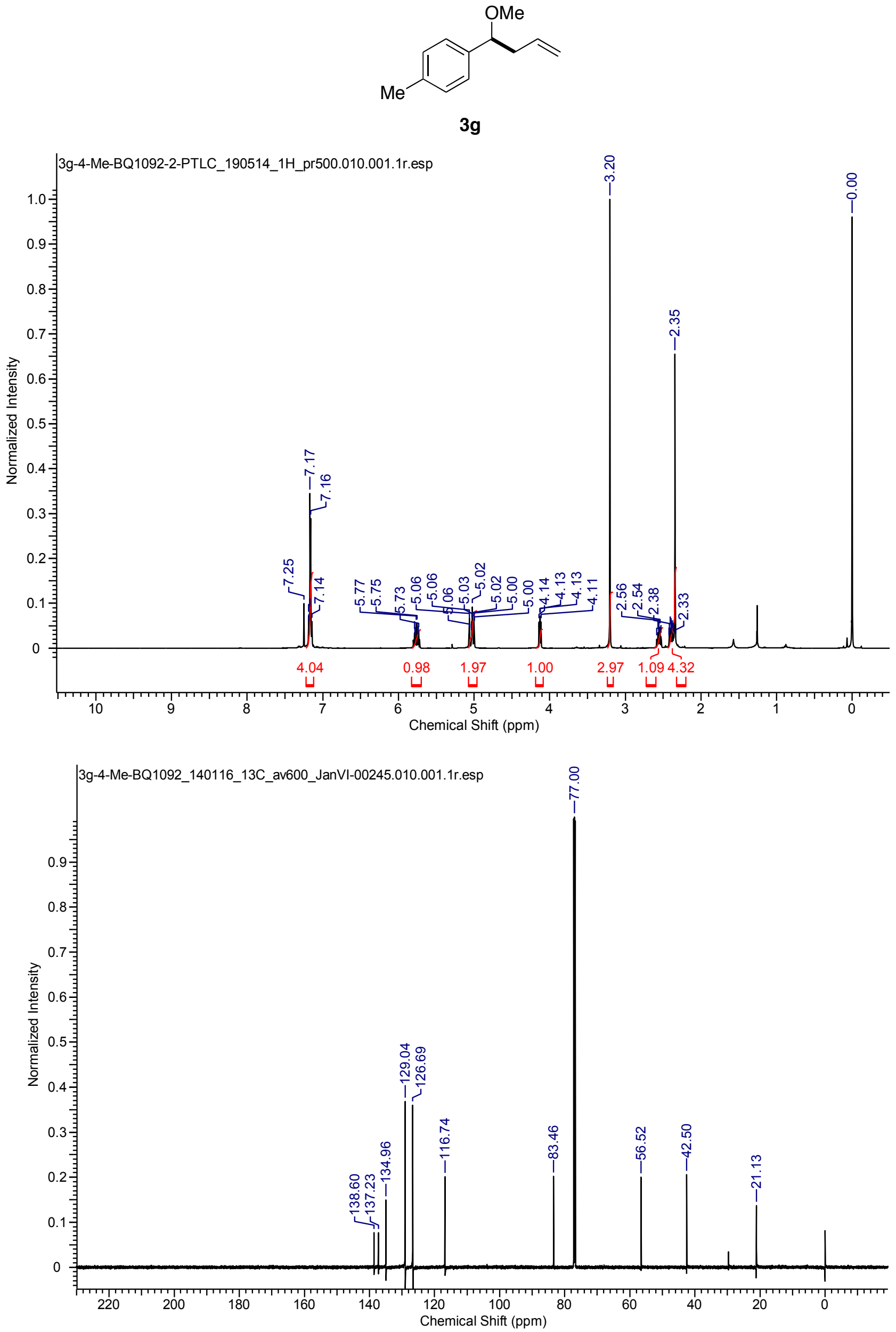
<smiles>C=CCC(OC)c1ccc(CO)cc1</smiles>

3h
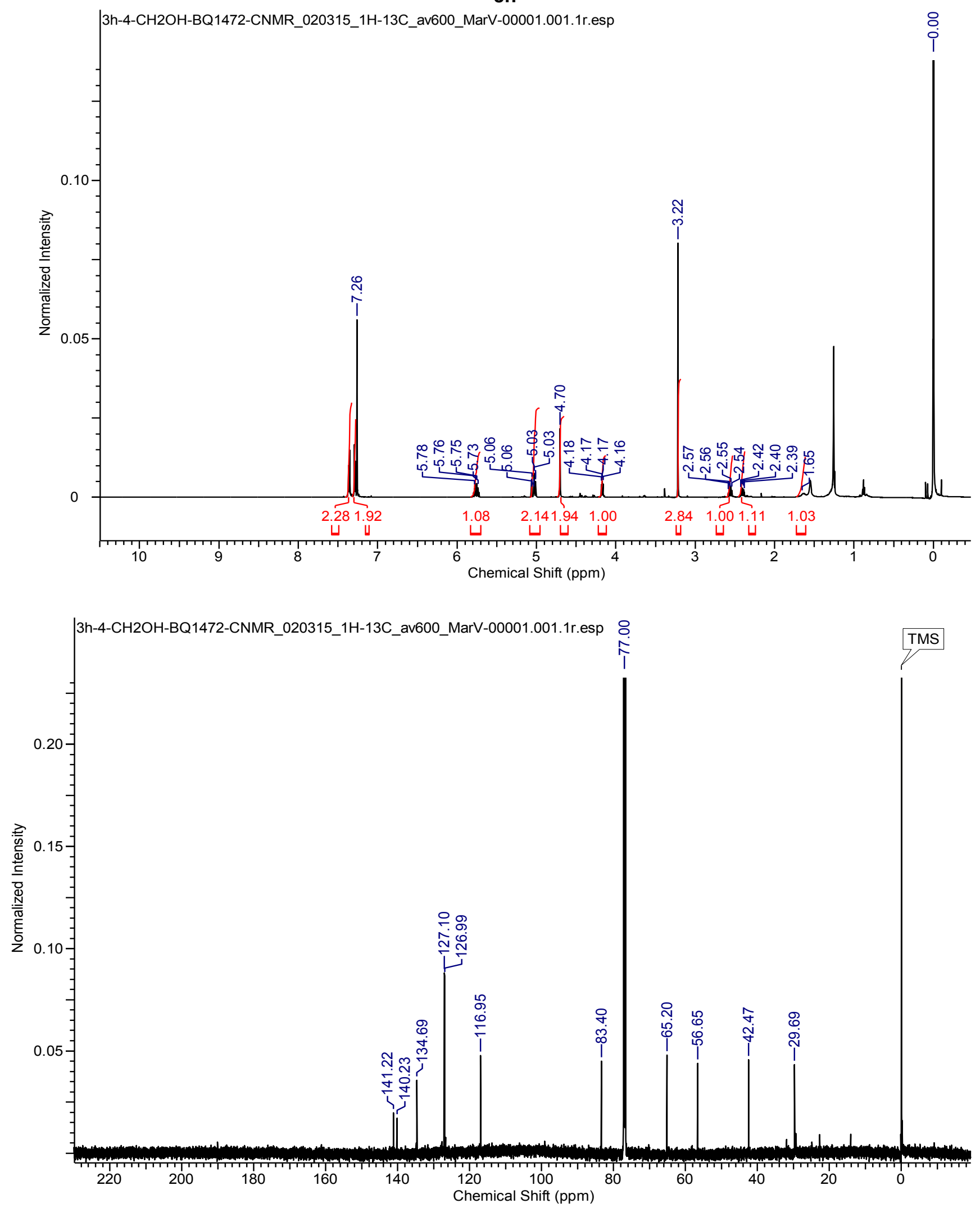
<smiles>C=CCC(OC)c1ccc(N(C)C)cc1</smiles>

3i
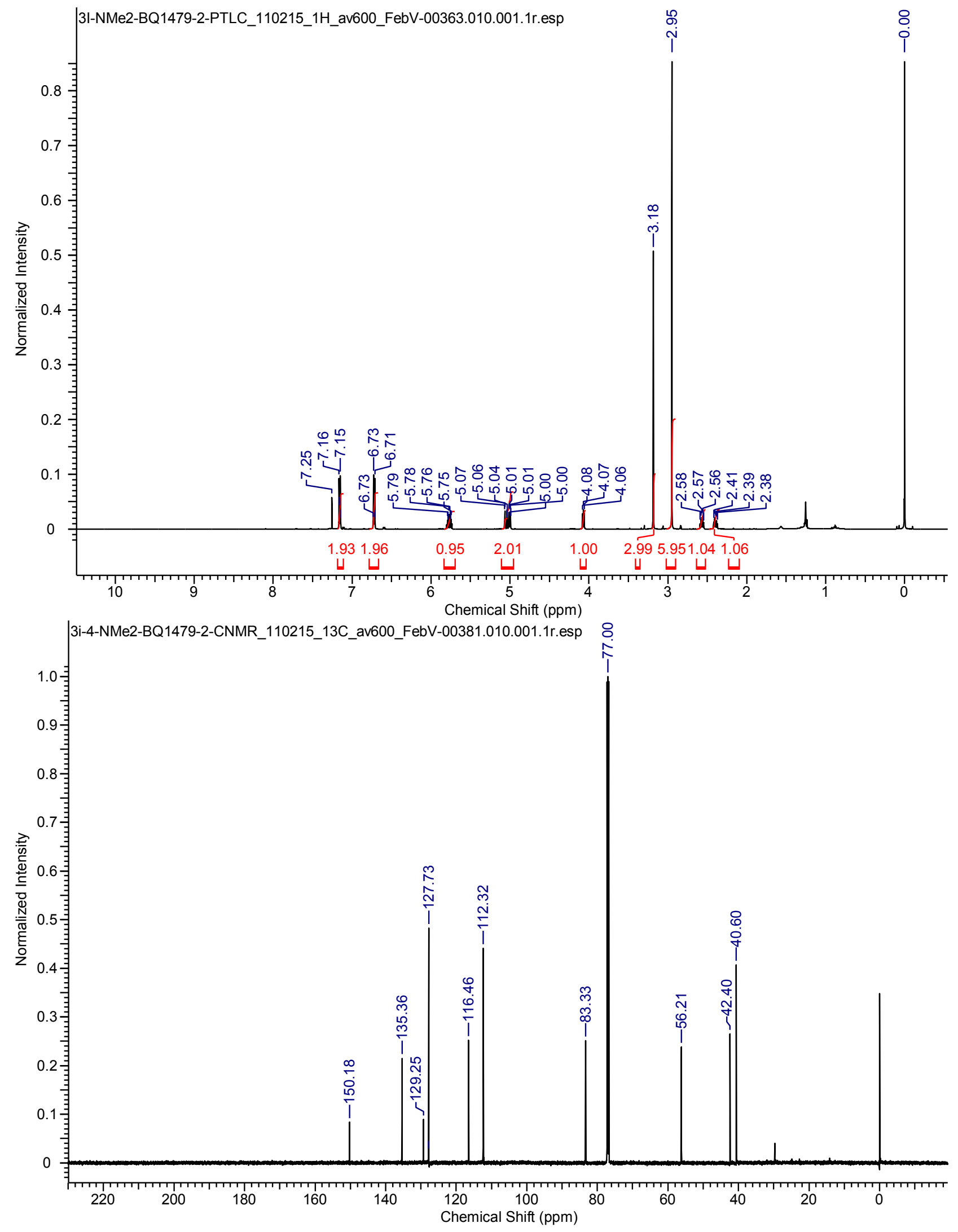
<smiles>C=CCC(OC)c1ccc(OC)cc1</smiles>

3j
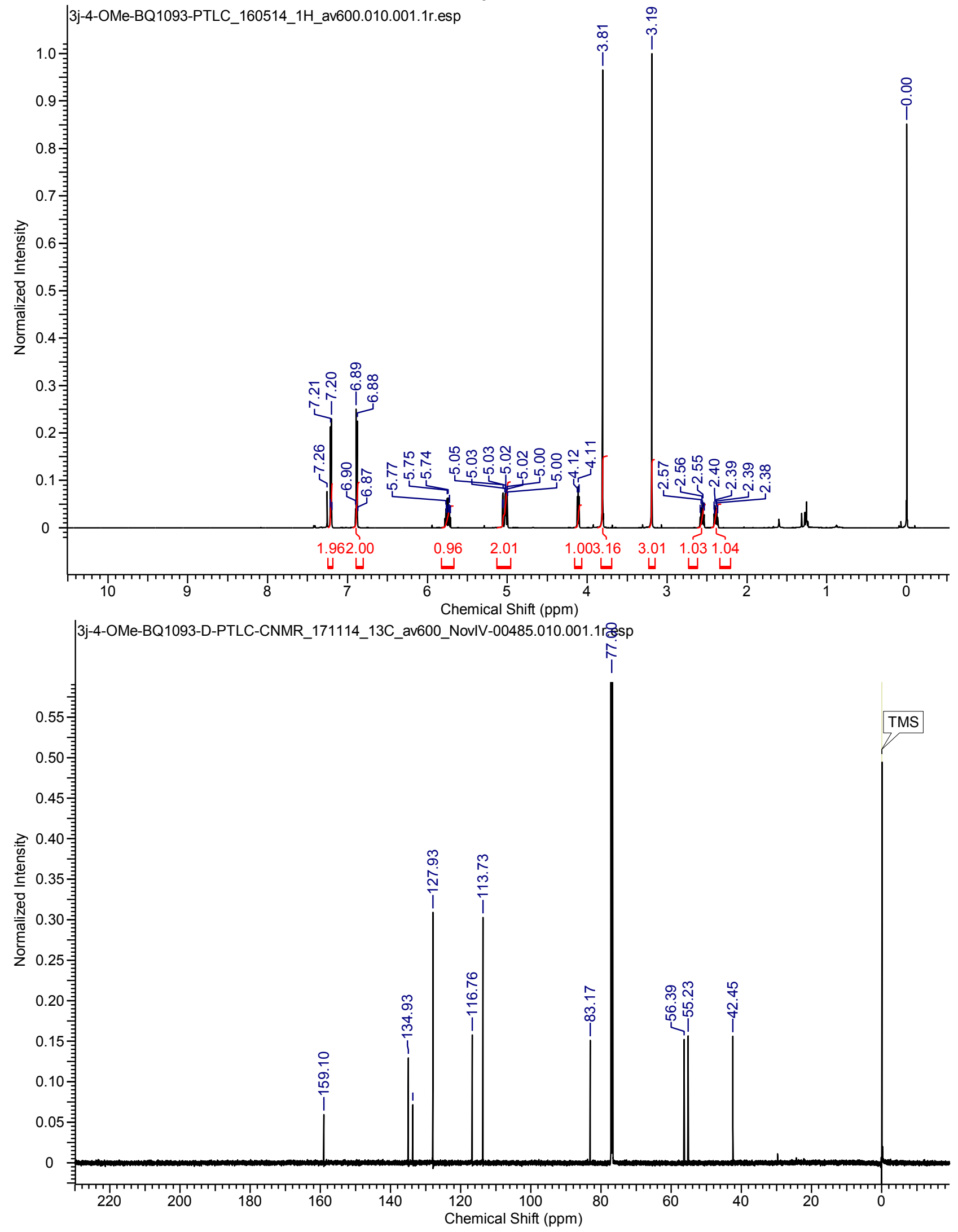


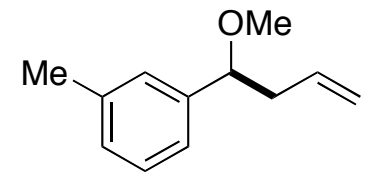

3k
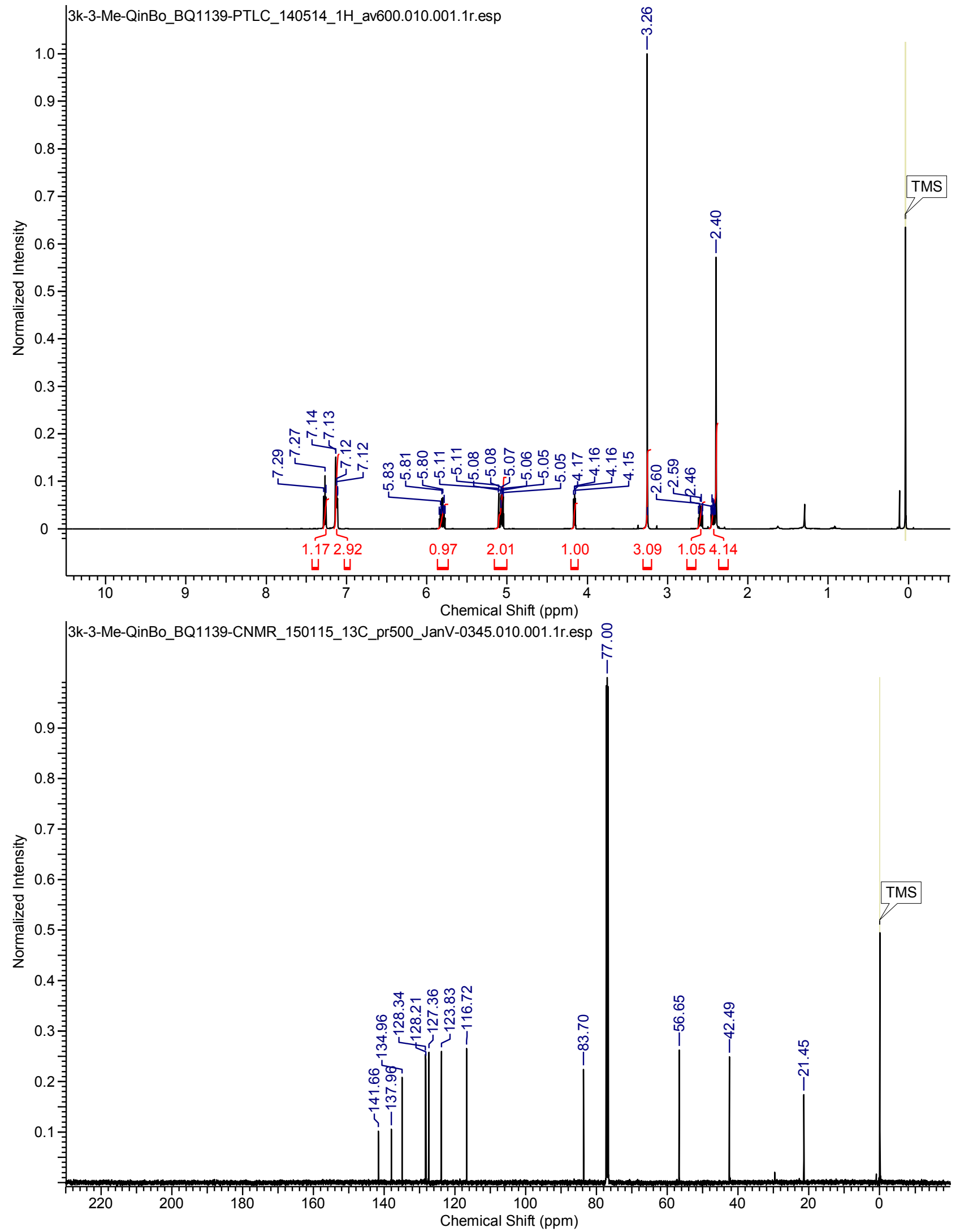


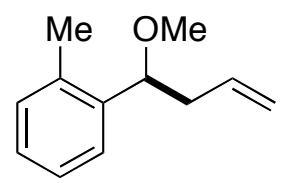

3I
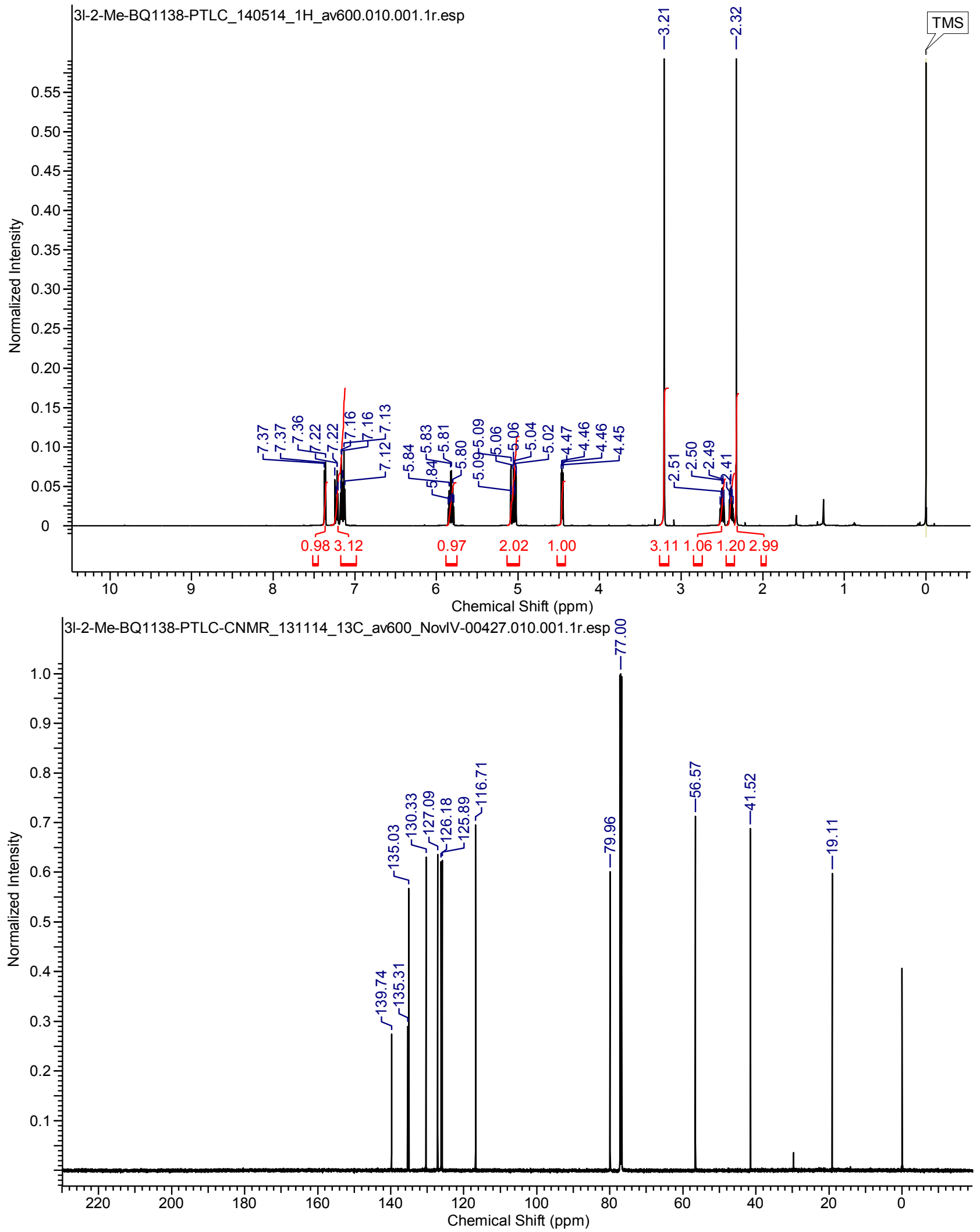


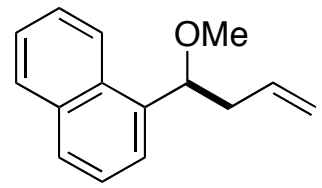

$3 \mathrm{~m}$
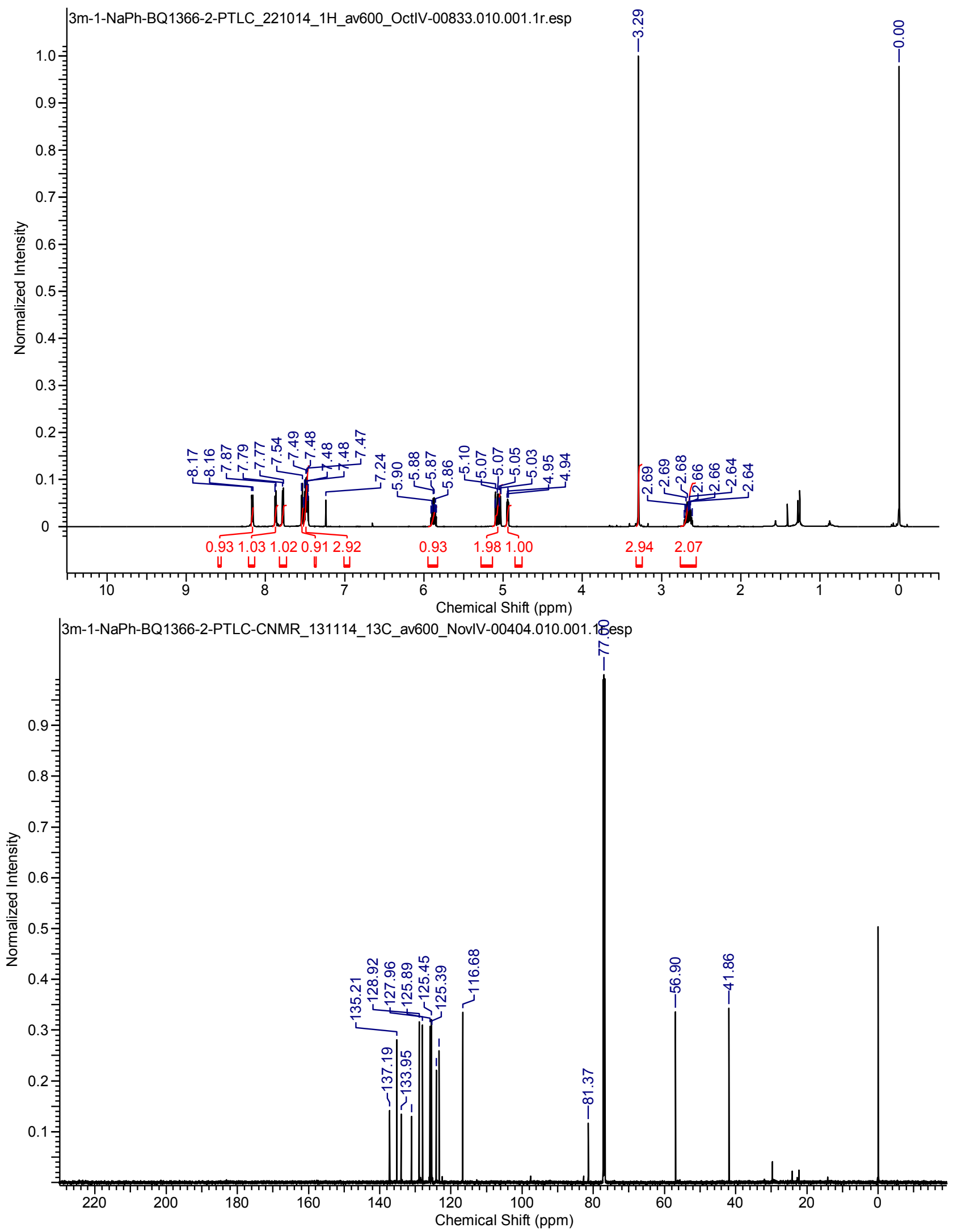
<smiles>C=CCC(OC)c1ccc2ccccc2c1</smiles>

3n
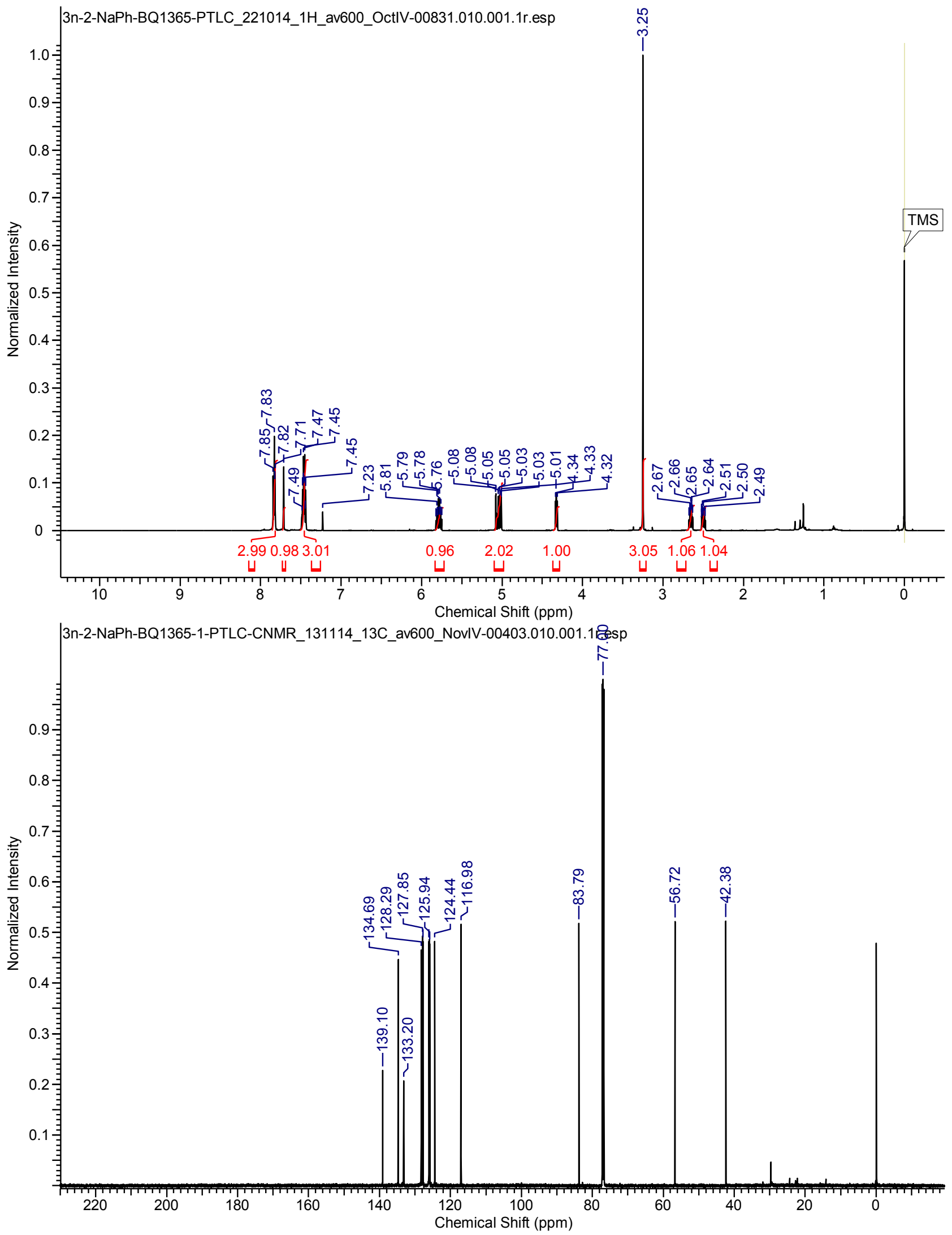


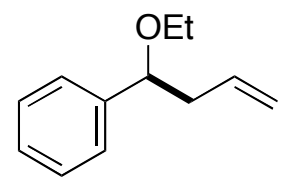

30
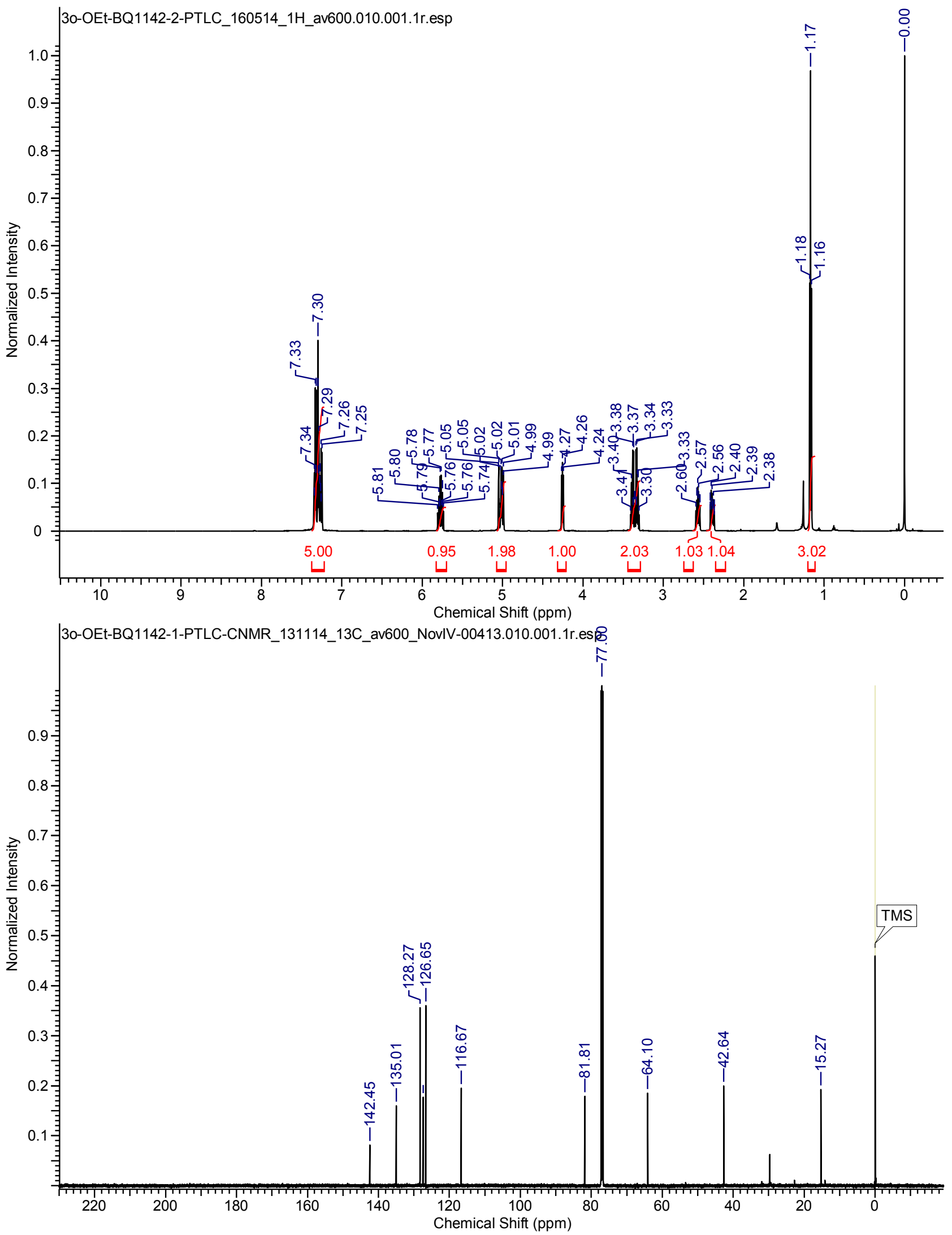


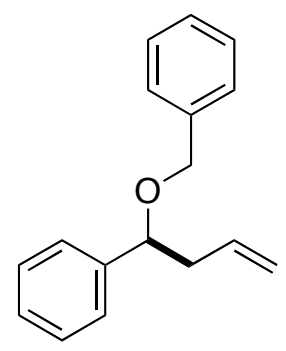

$3 p$
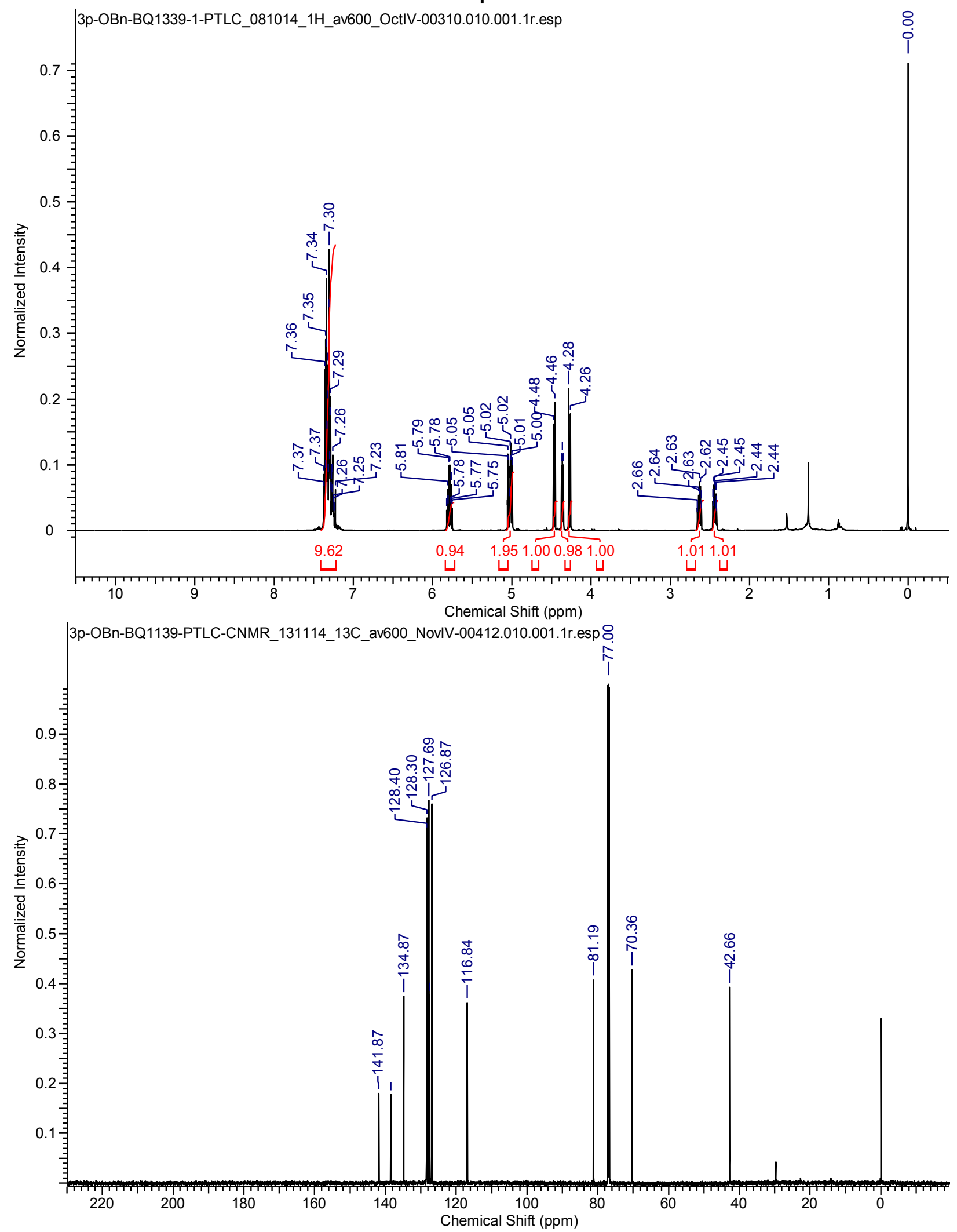


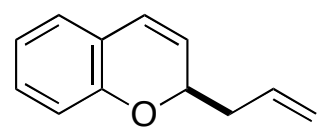

$3 q$
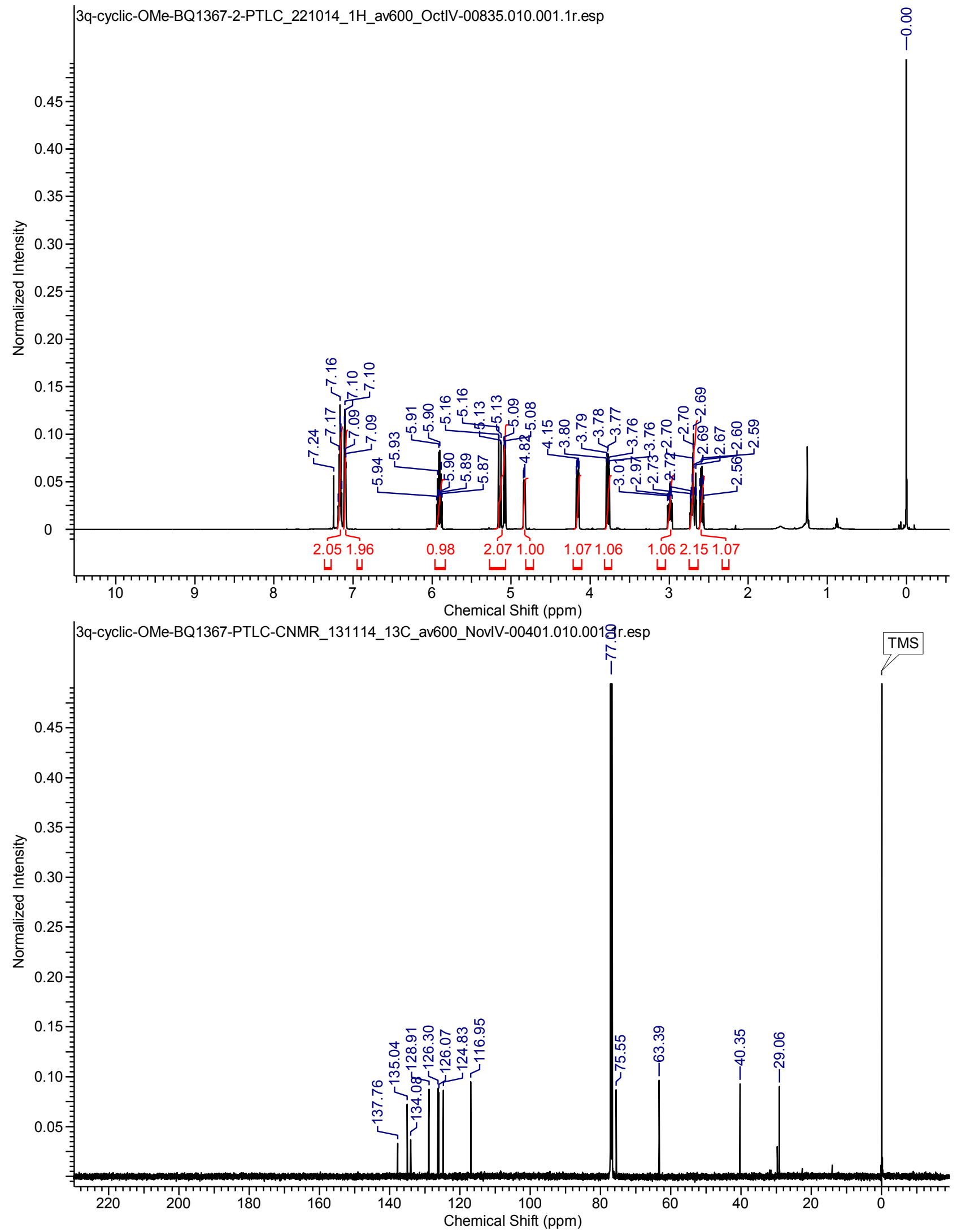


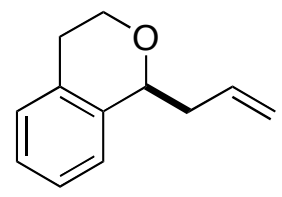

$3 r$
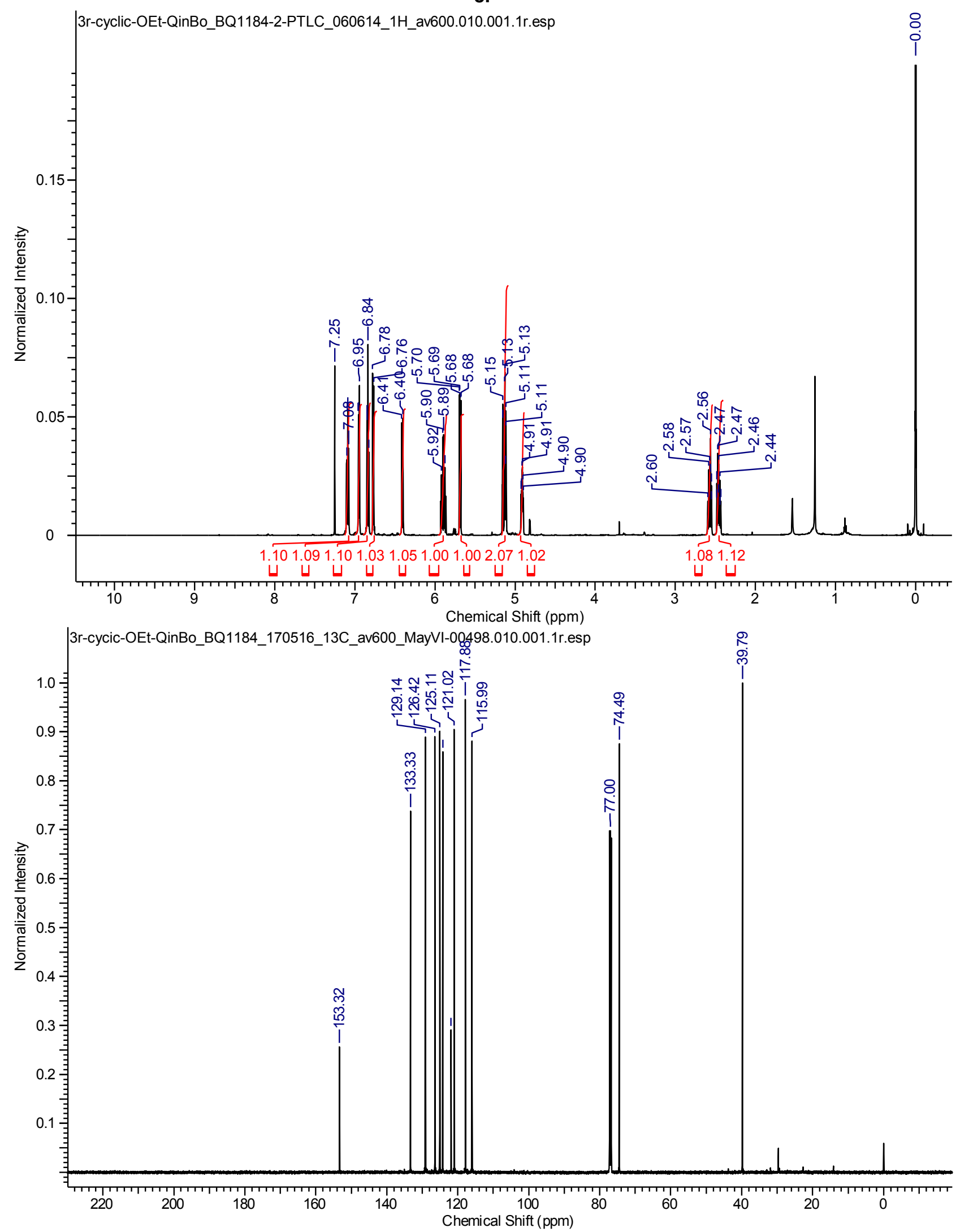


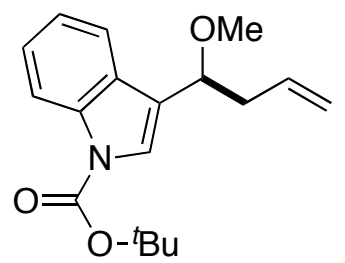

3 s
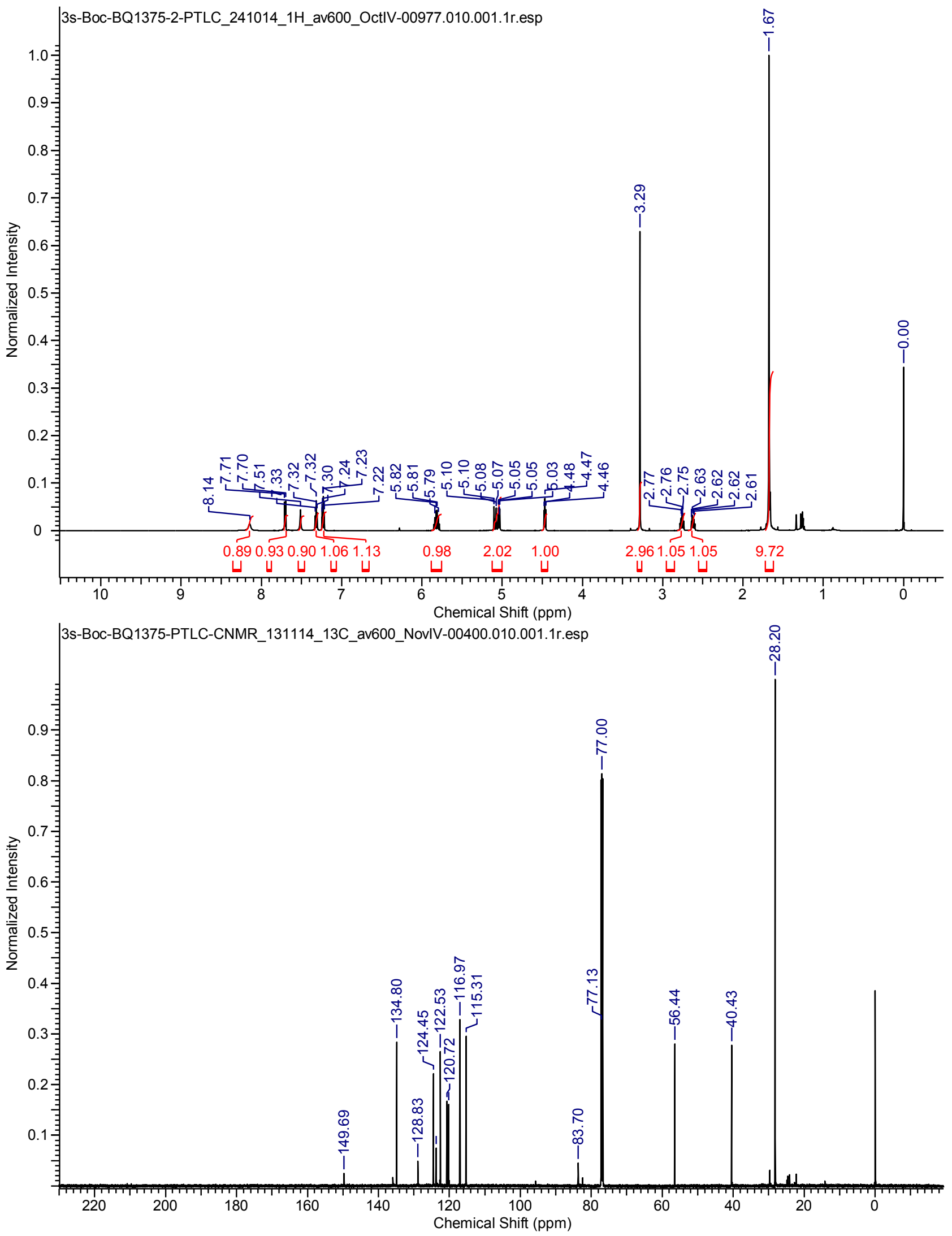


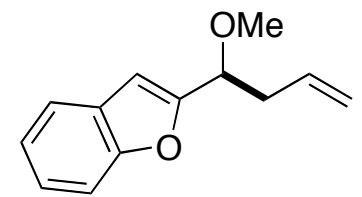

3t
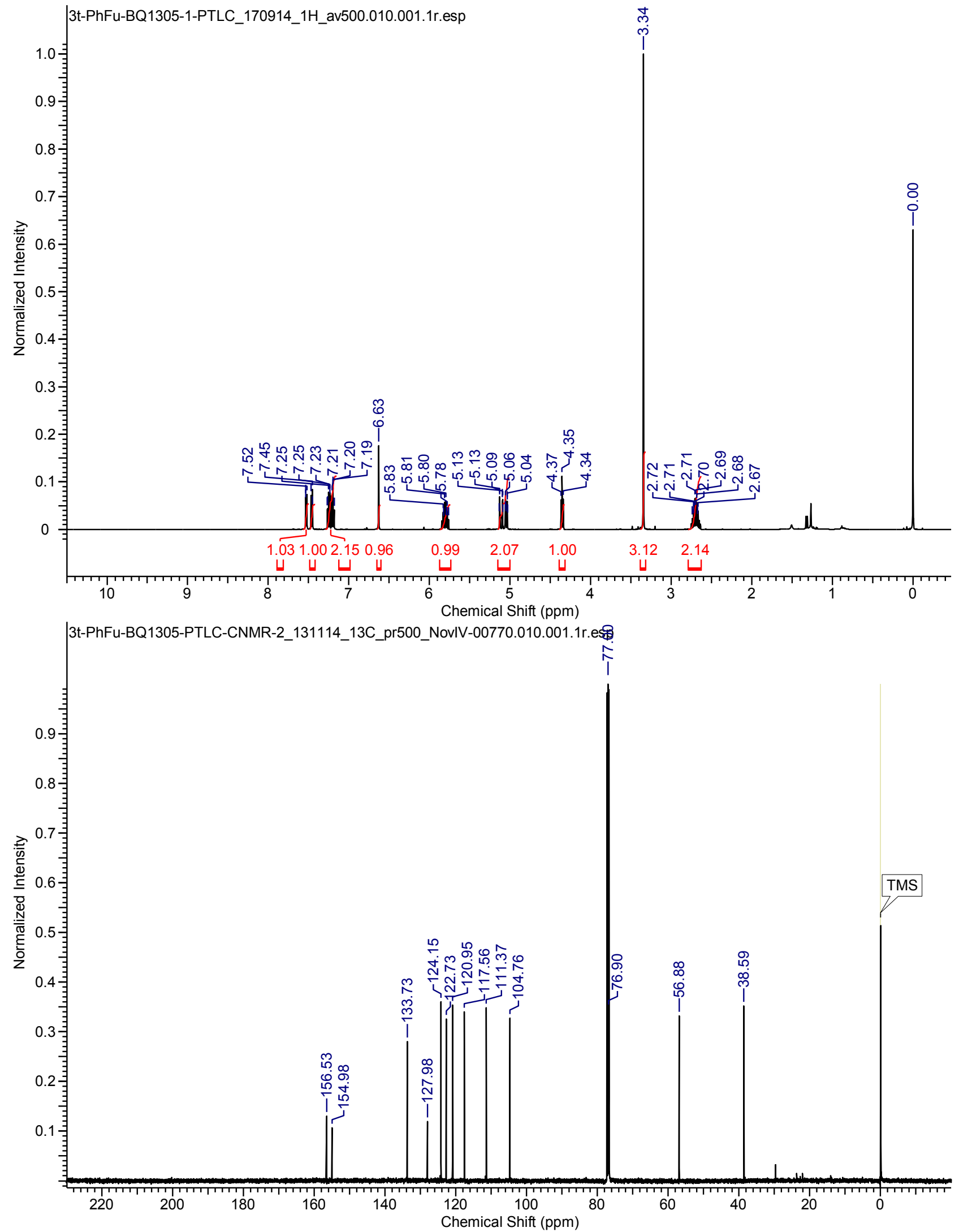


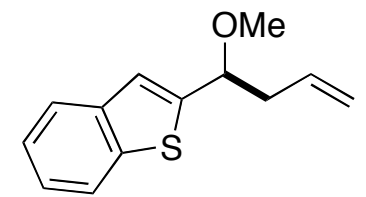

$3 u$
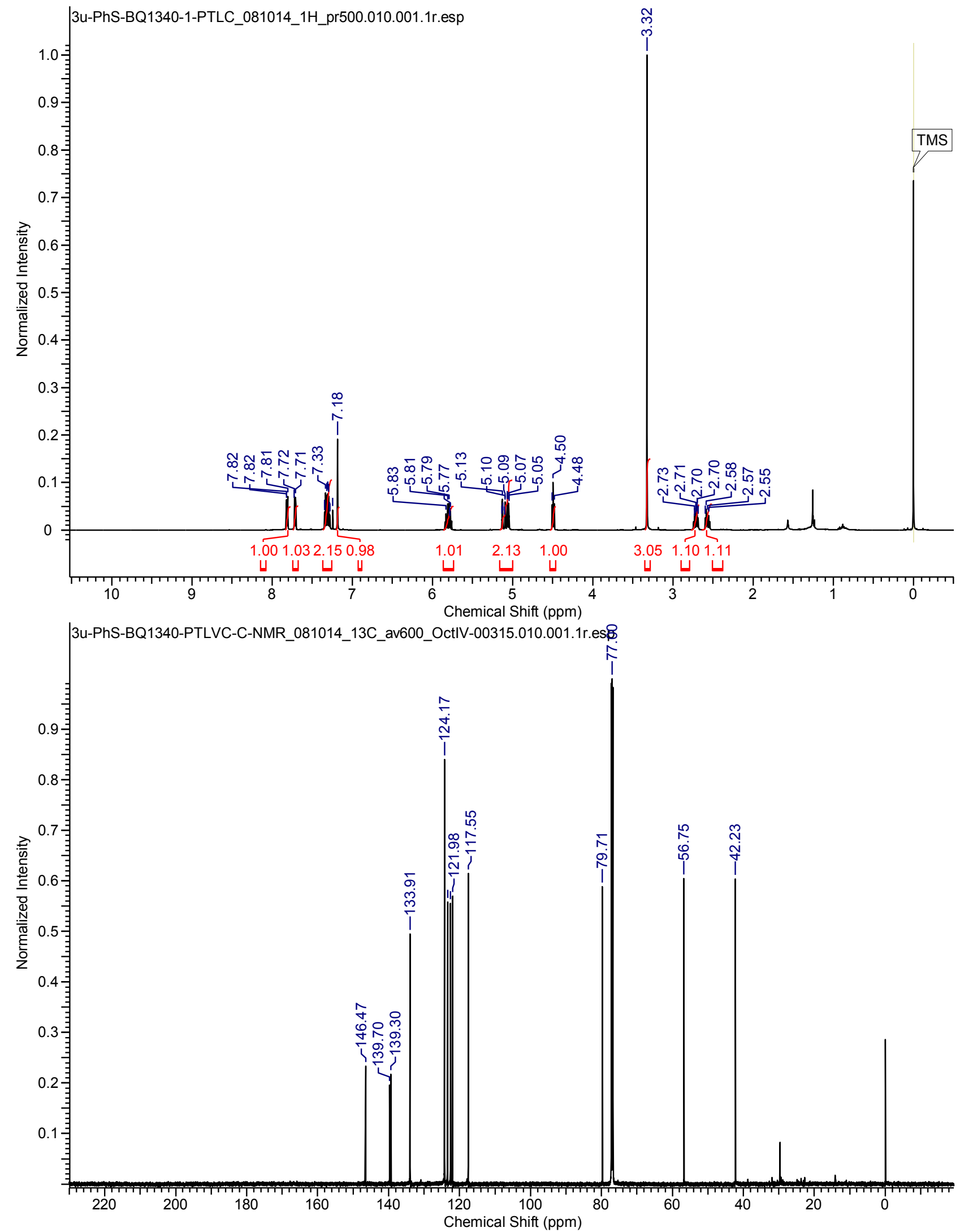


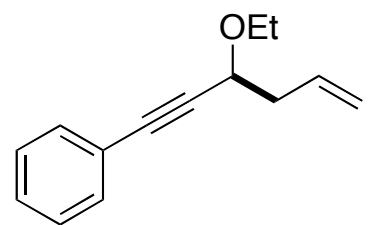

3v
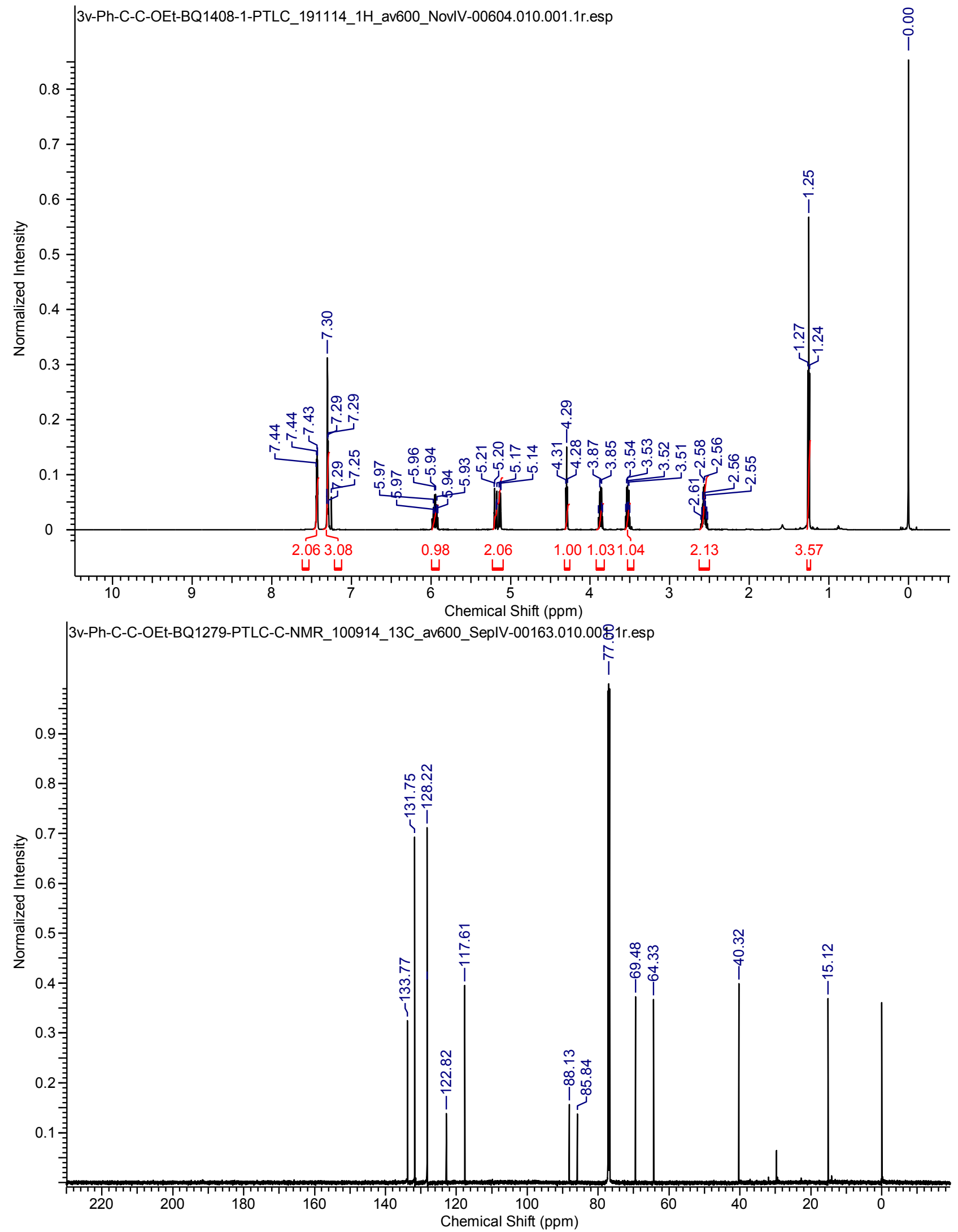


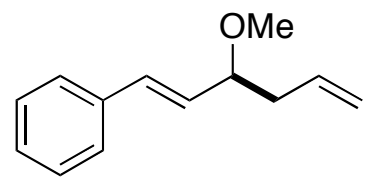

3w
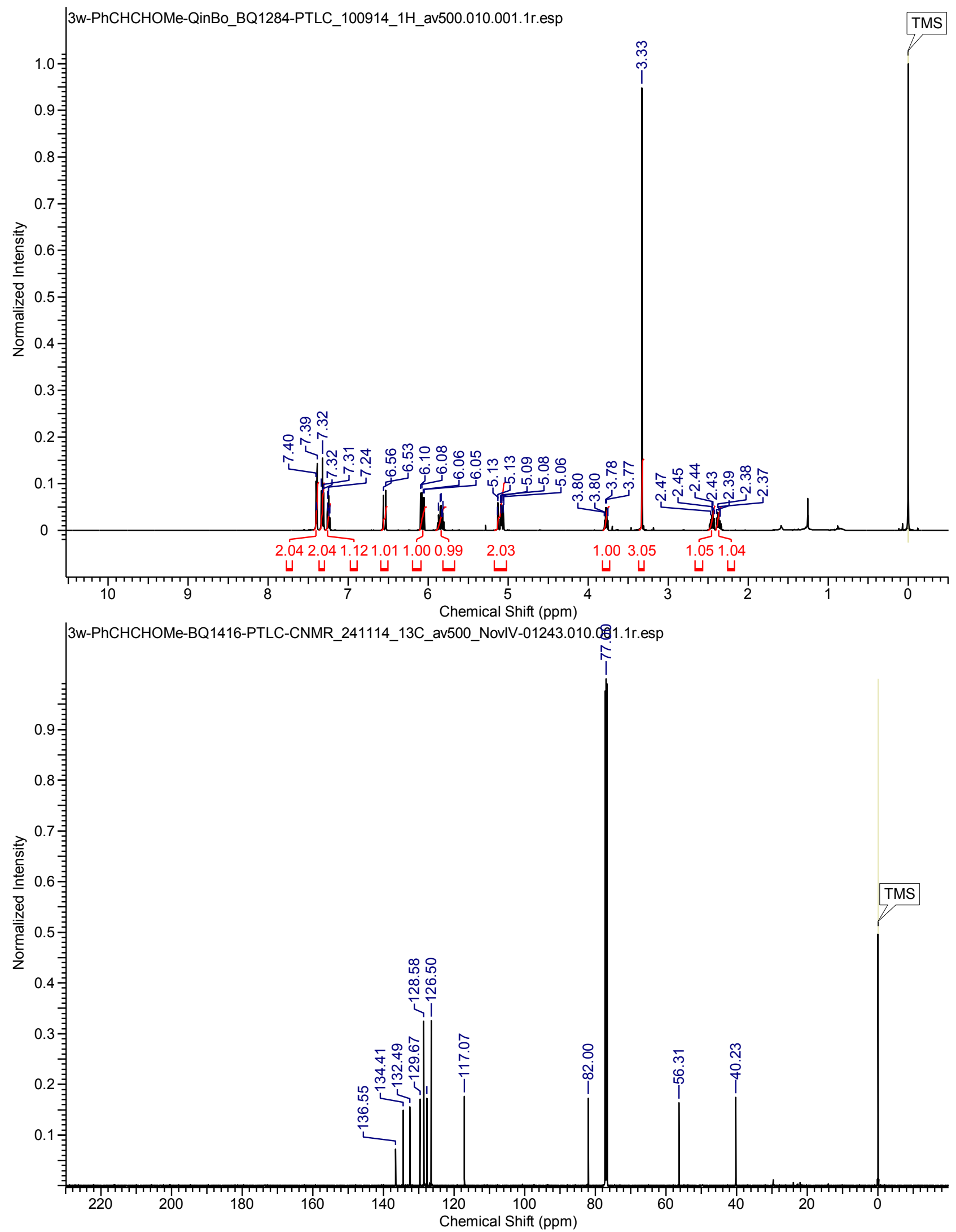


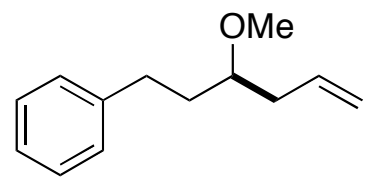

$3 x$
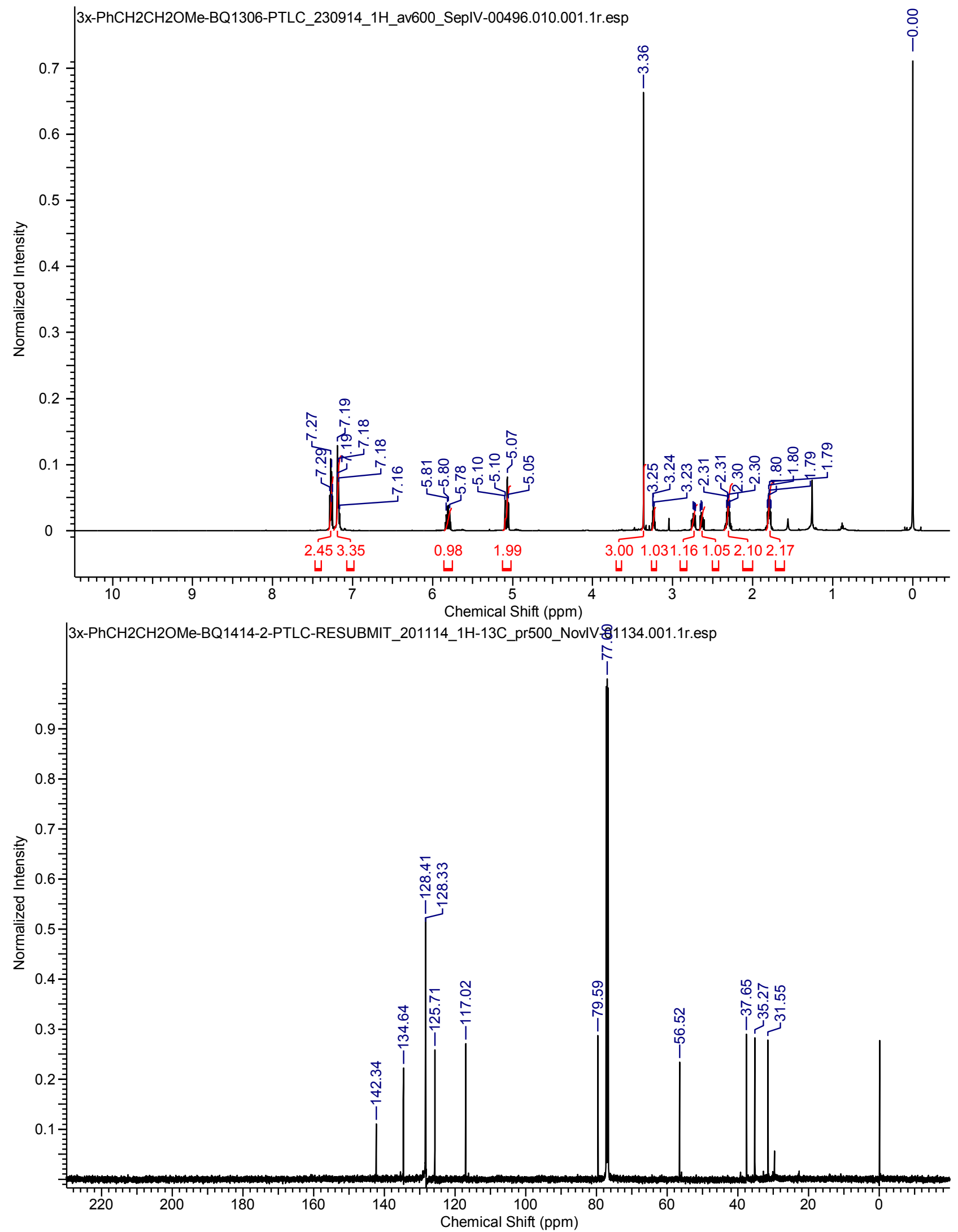


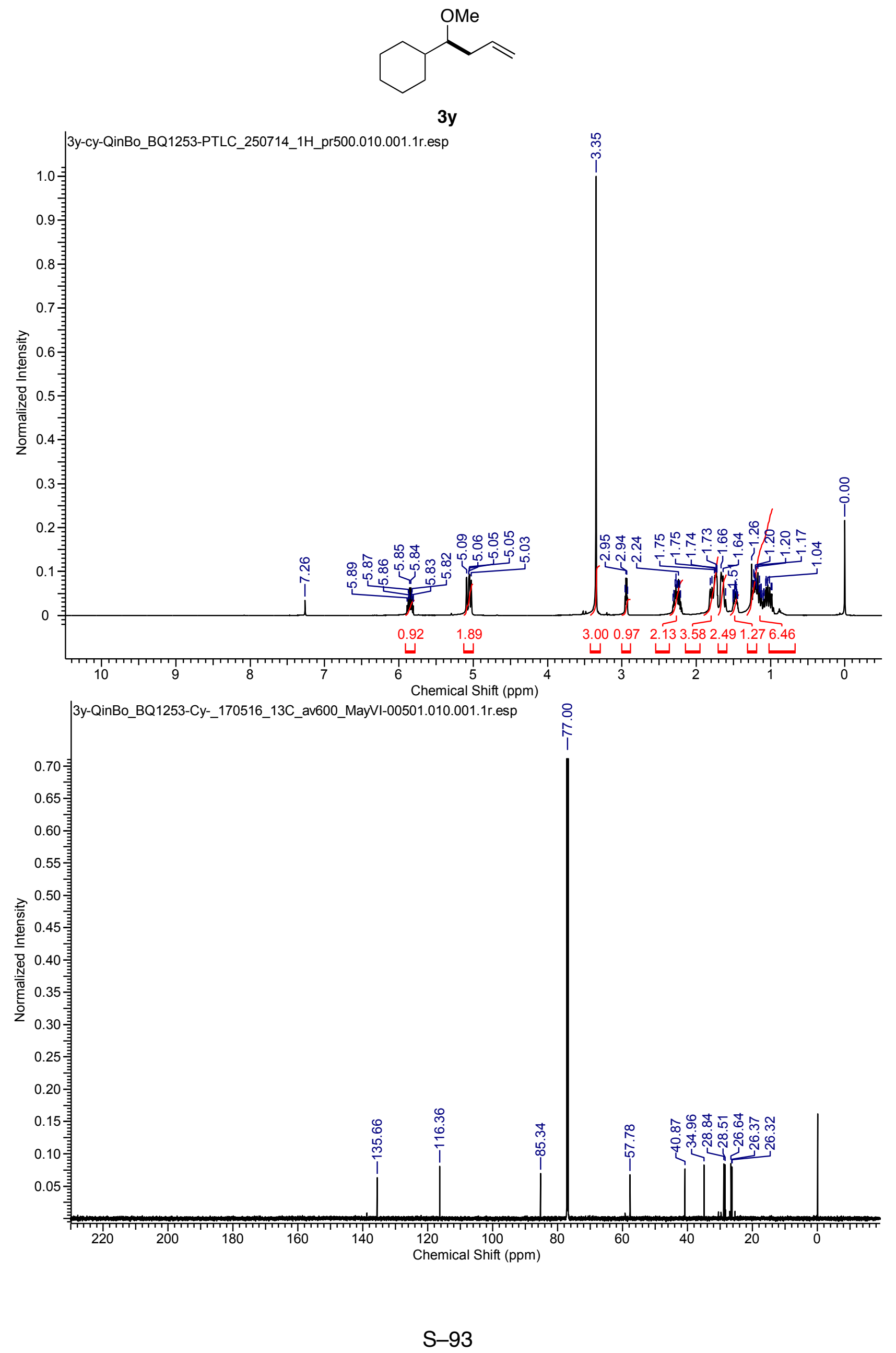




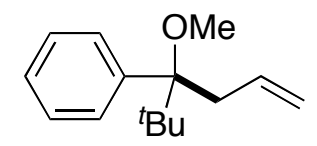

$3 z$
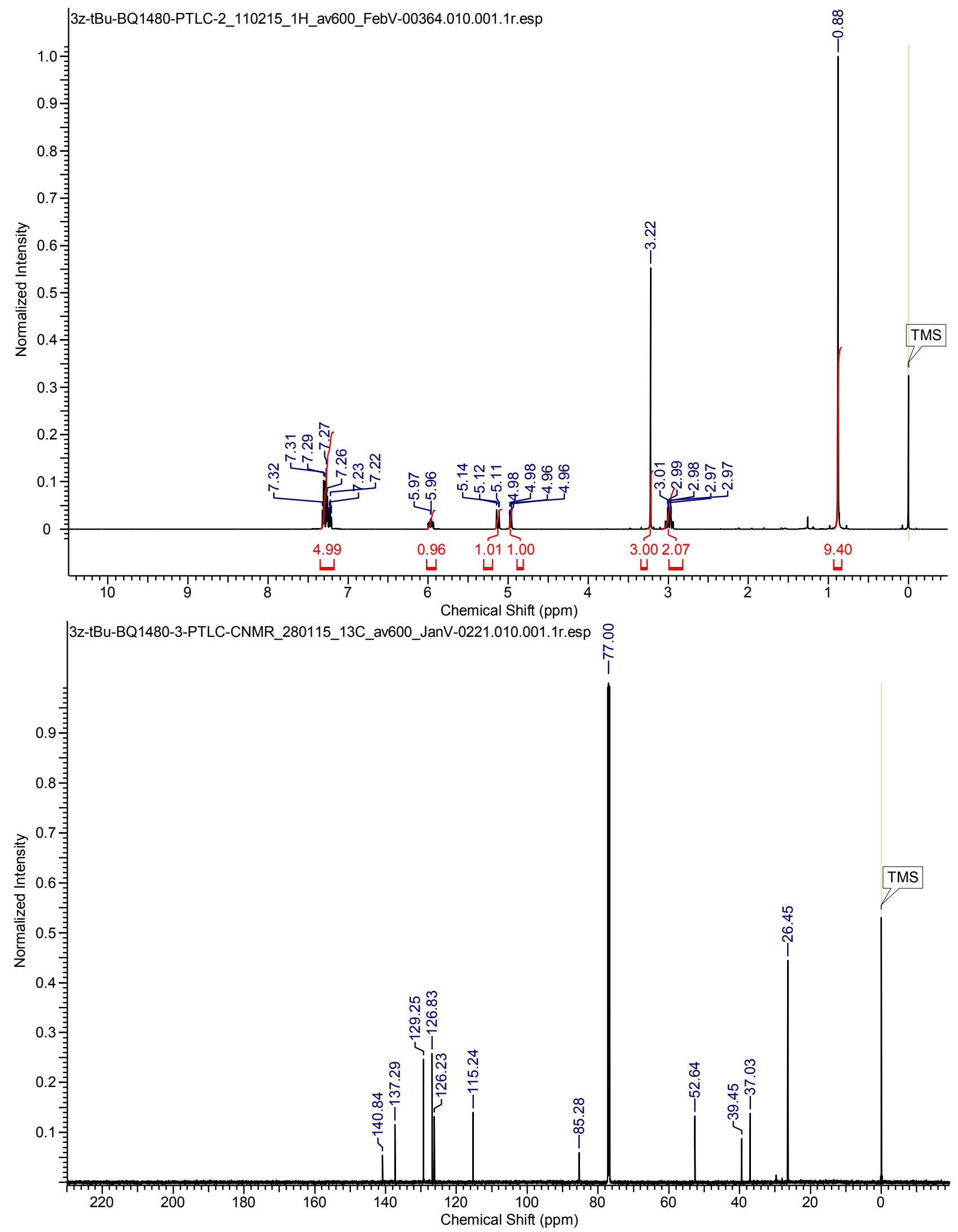


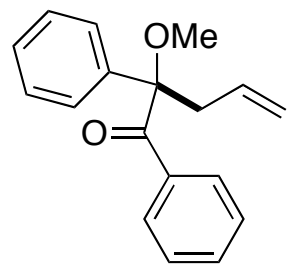

$3 z^{\prime}$
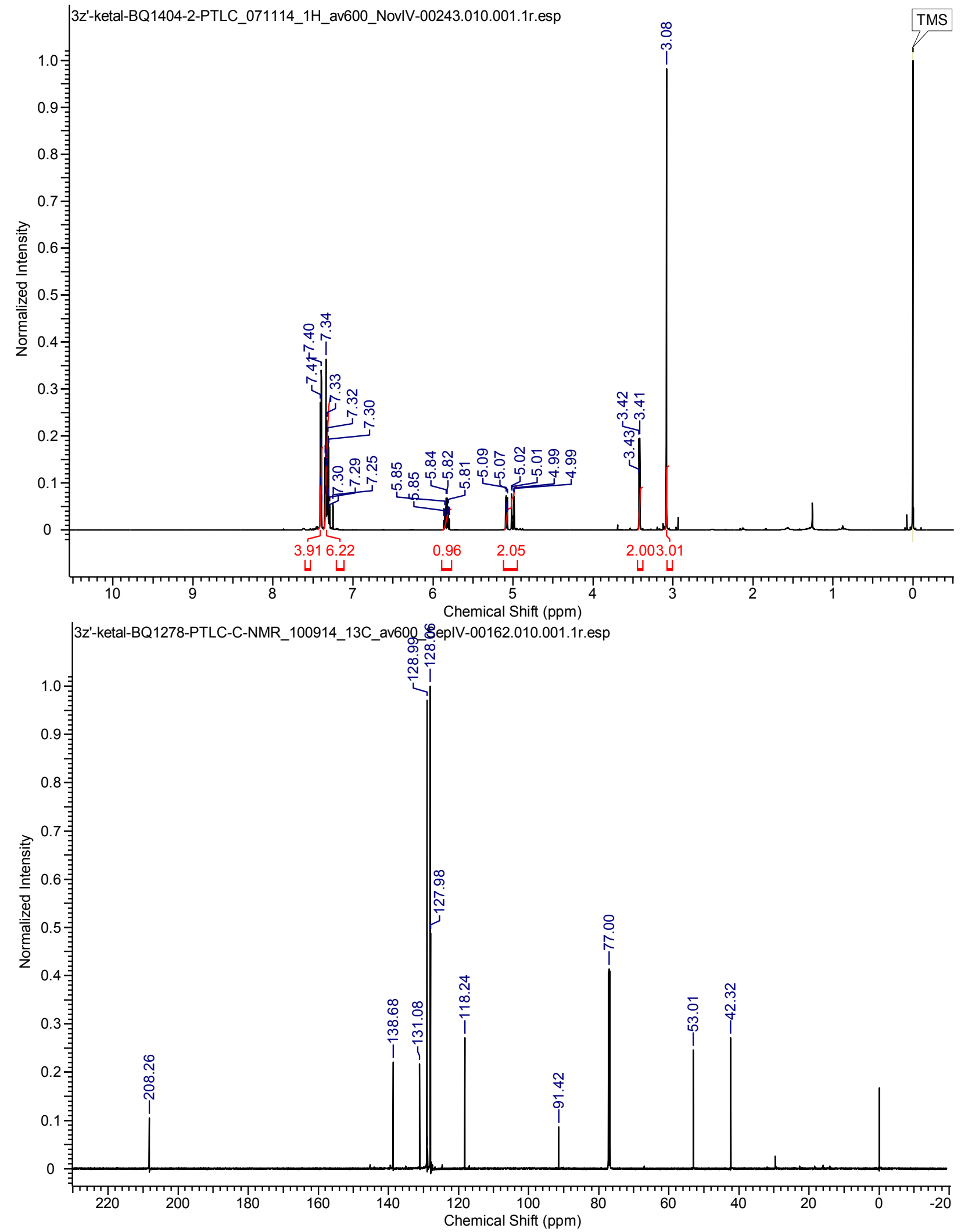

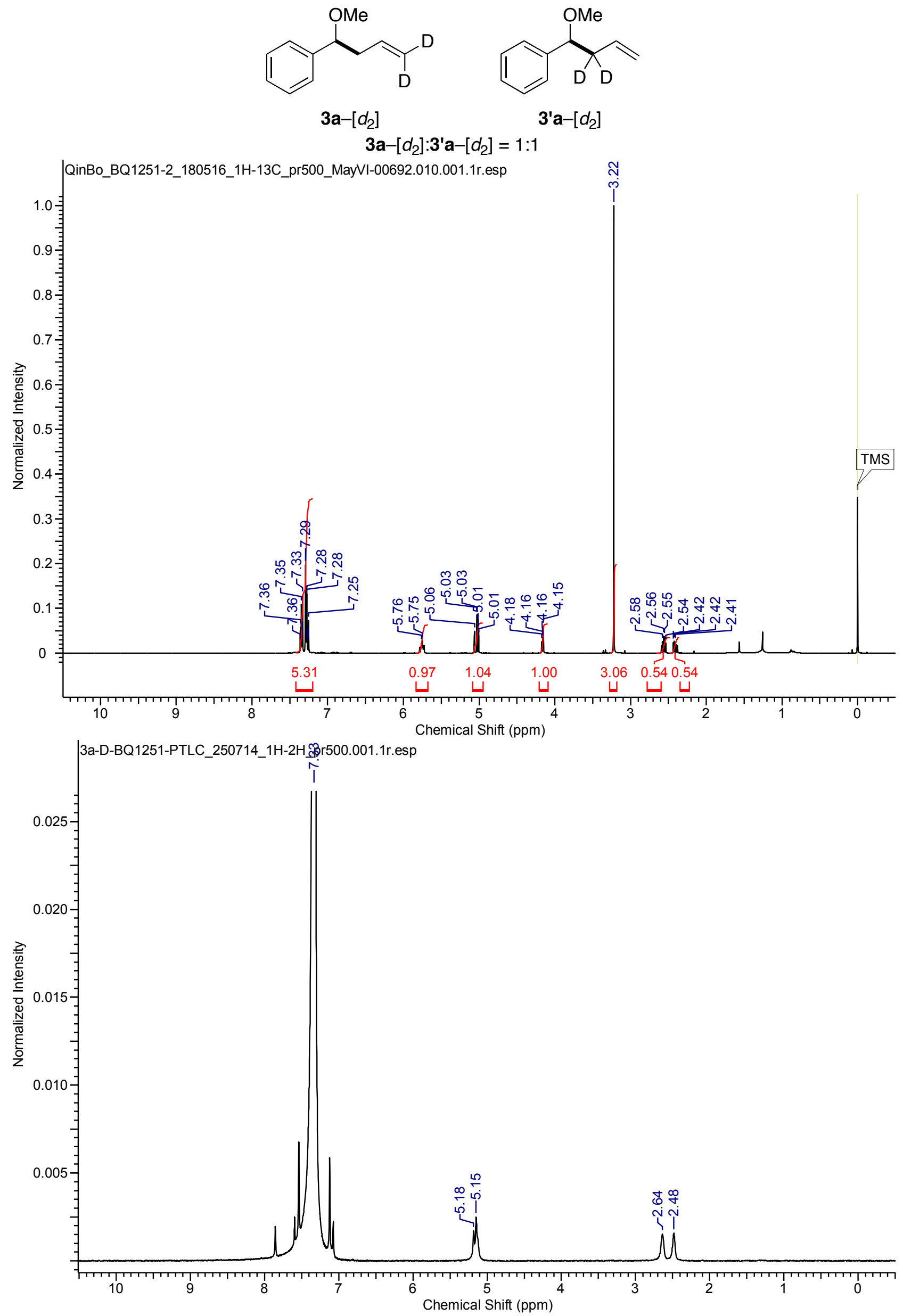


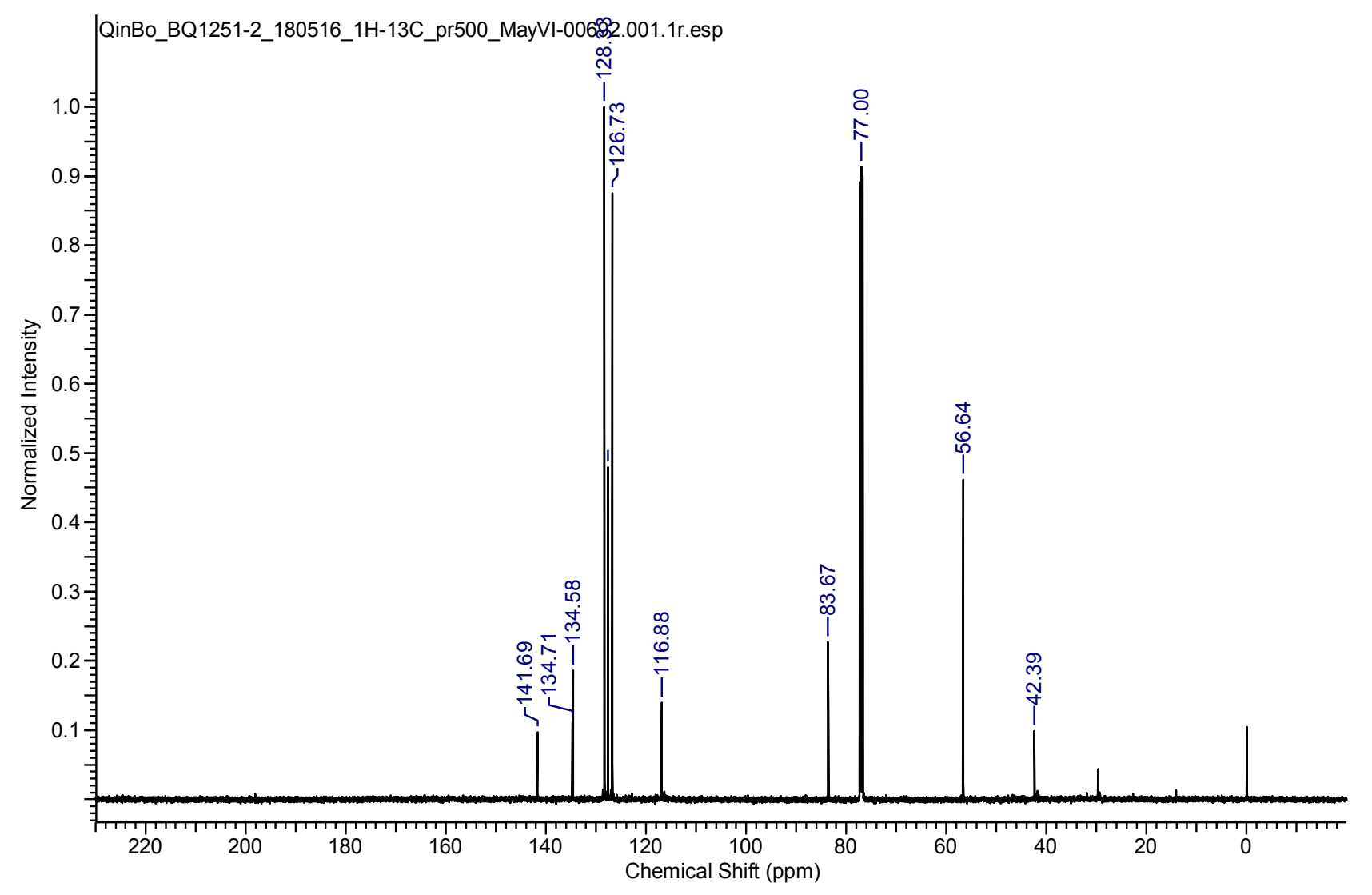




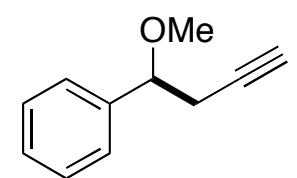

$15 a$<smiles>C=CC(OC)c1ccccc1</smiles>

$16 a$

$15 a: 16 a=49: 1$
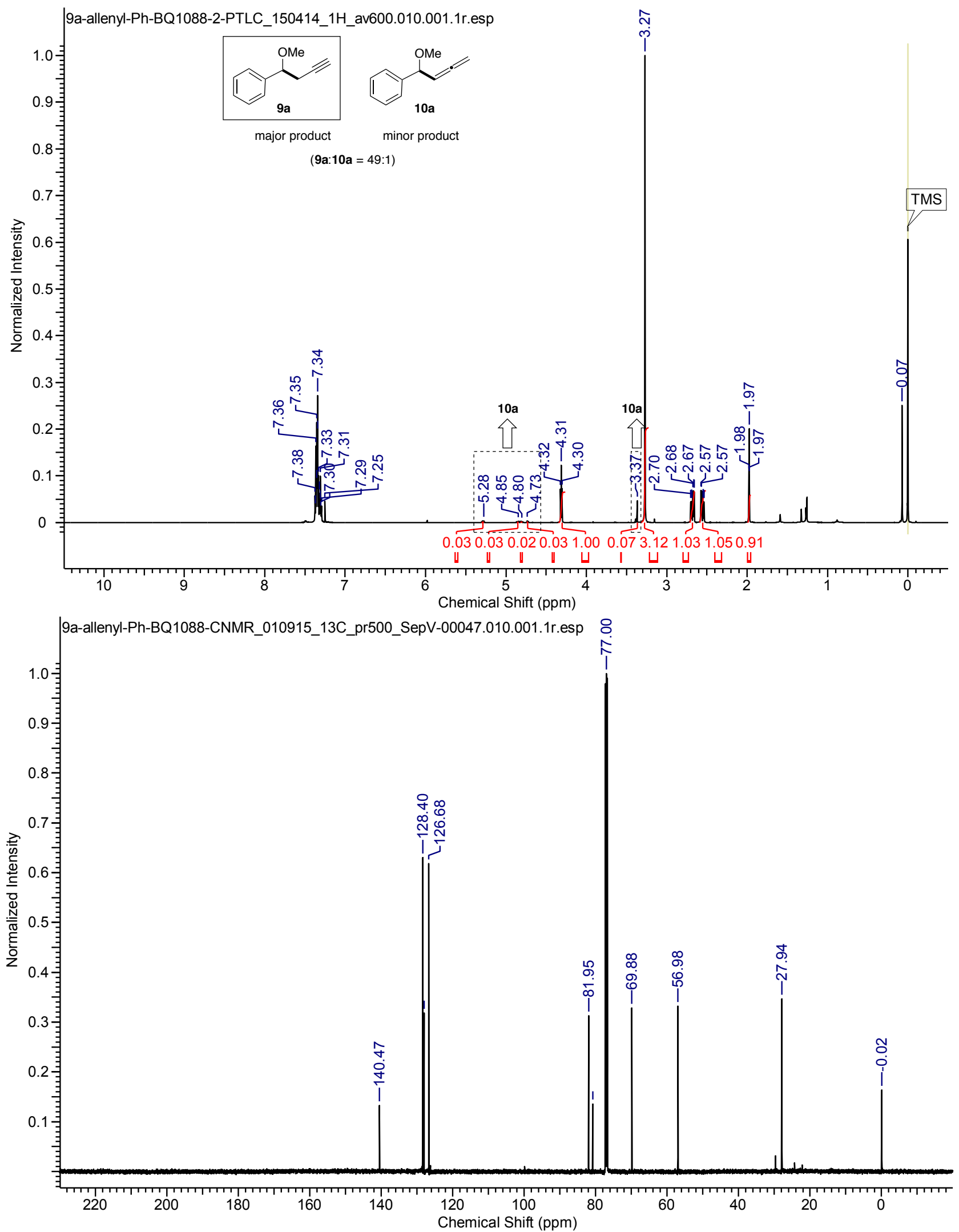


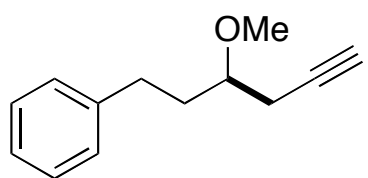

$15 x$<smiles>C=C=CC(CCc1ccccc1)OC</smiles>

$16 x$

$15 x: 16 x=>30: 1$
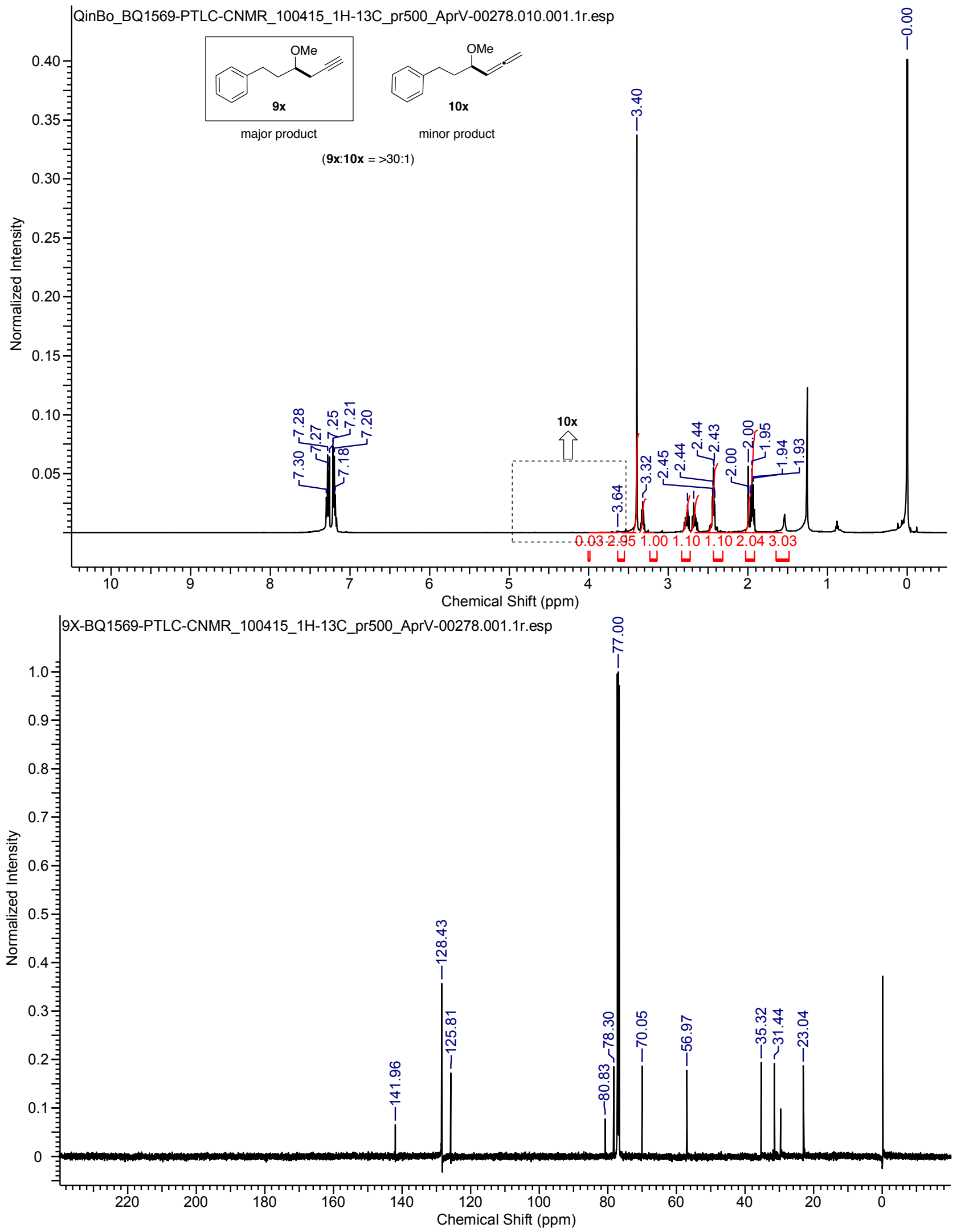
<smiles>CC(C)(C)OP(=O)(O)Oc1c(-c2cc(C(C)(C)C)cc(C(C)(C)C)c2)cc2ccccc2c1-c1c(OP(=O)(OC(C)(C)C)OC(C)(C)C)cc(C(C)(C)C)cc1-c1cc(C(C)(C)C)cc(C(C)(C)C)c1</smiles>

(R)-17
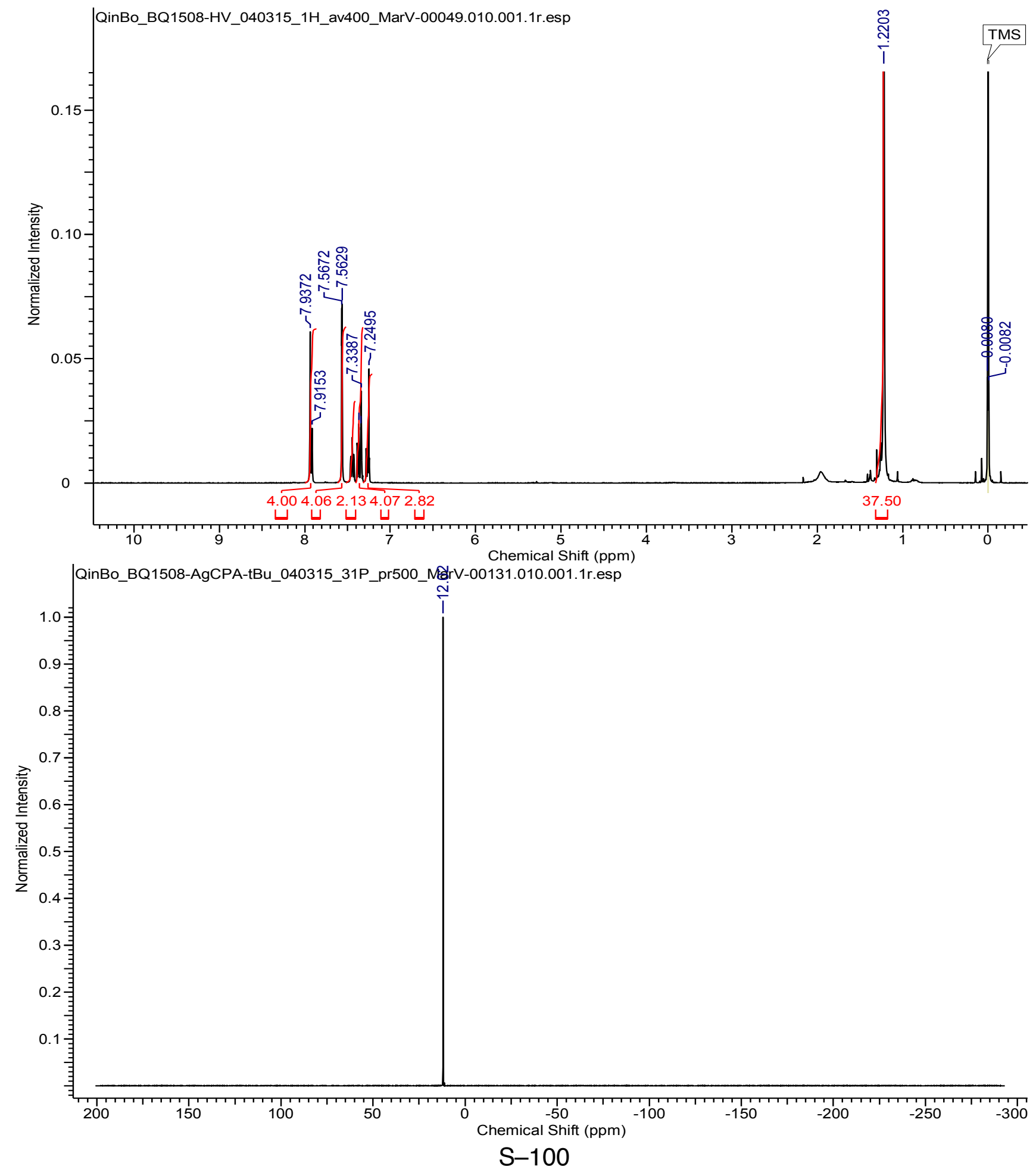


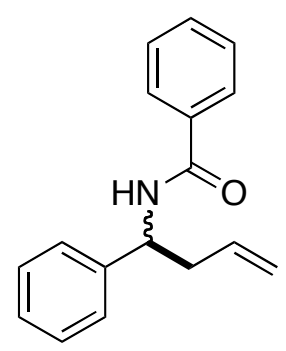

rac-13
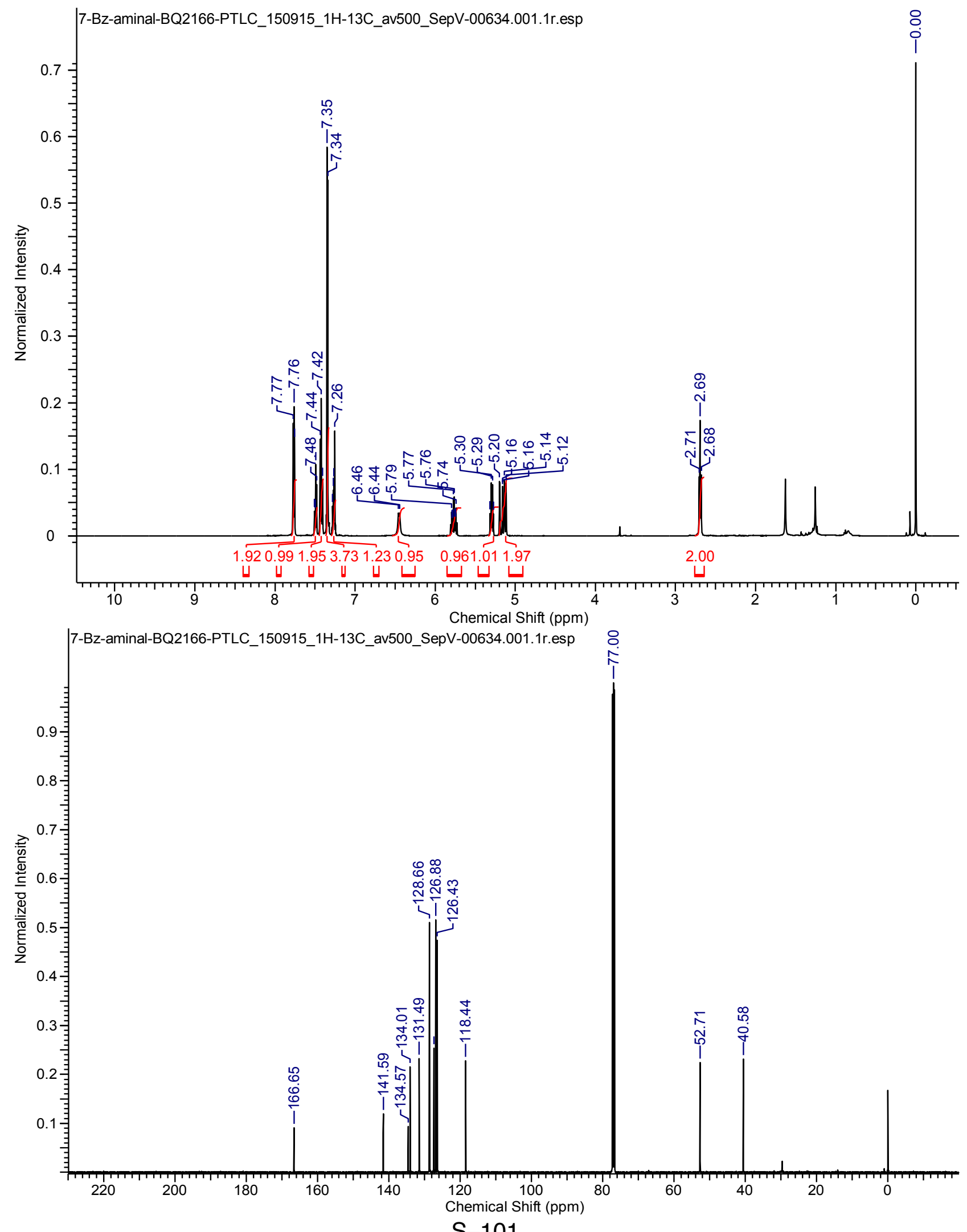


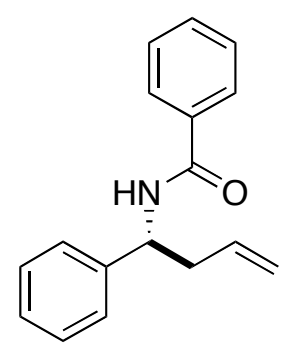

(R)-13

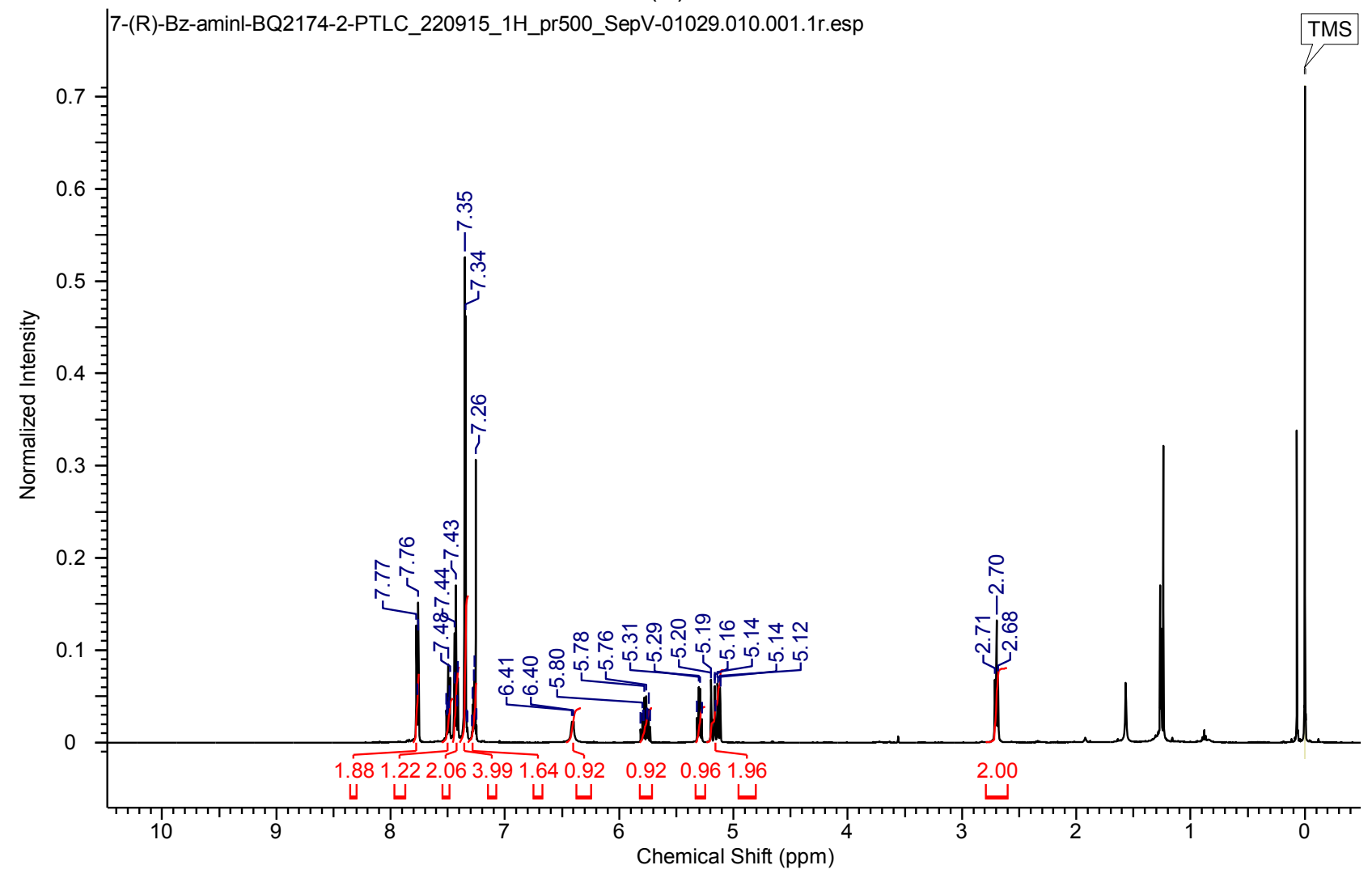


<smiles>C=CC[C@H](NC(=O)c1ccccc1)c1ccccc1</smiles>

$(R)-13$

\section{LabSolutions Analysis Report}

<Sample Information>

Sample Name : BQ2174-3

Sample ID $\quad \vdots$ BQ2174-3.Icd

Method Filename : wash.lcm

Batch Filename :

Vial \# $\quad: 1-1$

Date Acquired : 22/09/2015 16:57:02

Sample Type : Unknown

Date Processed : :22/09/2015 17:22:06

Acquired by : System Administrator

Processed by : System Administrator

\section{$<$ Chromatogram >}

$\mathrm{mV}$

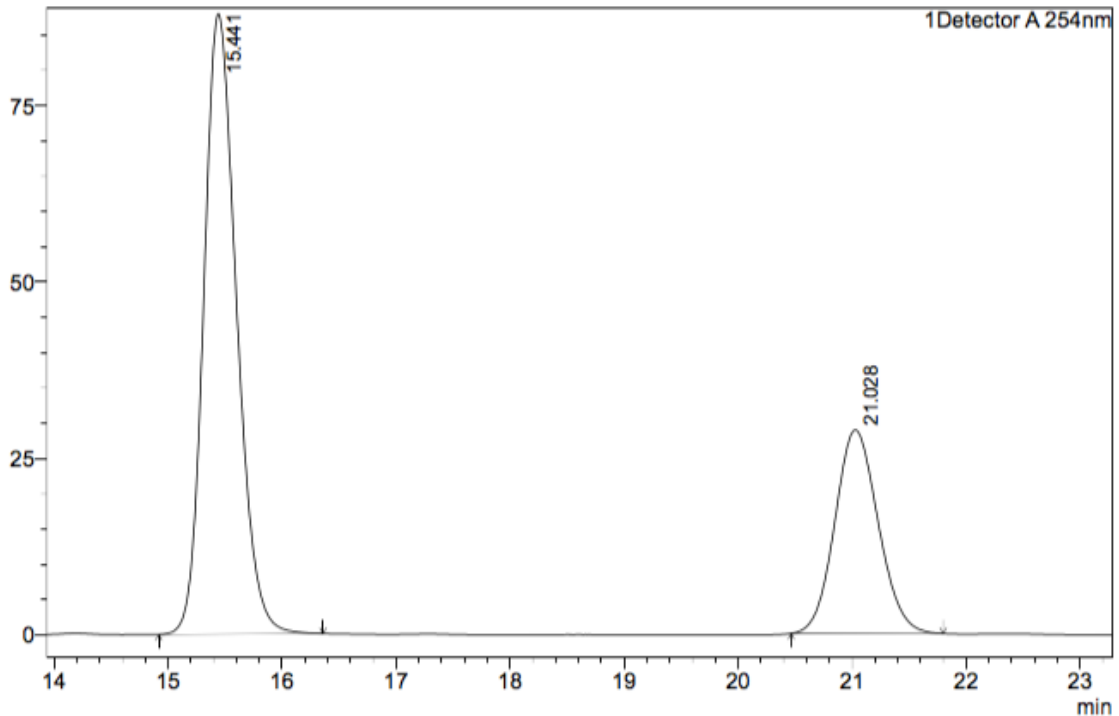

\section{<Peak Table>}

Detector A 254nm

\begin{tabular}{|c|c|c|c|c|c|c|c|}
\hline Peak\# & Ret. Time & Area & Height & Conc. & Unit & Mark & Name \\
\hline 1 & 15.441 & 1751303 & 87854 & 70.051 & & $M$ & \\
\hline 2 & 21.028 & 748731 & 28640 & 29.949 & & $M$ & \\
\hline Total & & 2500034 & 116494 & & & & \\
\hline
\end{tabular}


<smiles>C=CC[C@H](NC(=O)c1ccccc1)c1ccccc1</smiles>

rac-13

\section{LabSolutions Analysis Report}

\section{<Sample Information>}

Sample Name : BQ1786-IPROH-HEX-90-10-1.0-1

Sample ID $\quad:$ BQ1786-IPROH-HEX-90-10-1.0-1.Icd

Method Filename : wash.Icm

Batch Filename

Vial \#

Injection Volume : 20 uL

Date Acquired : : :19/05/2015 14:41:08

Sample Type : Unknown

Date Processed : : 19/05/2015 15:10:38

Acquired by : : System Administrator Processed by : : System Administrator

$<$ Chromatogram $>$

$\mathrm{mV}$

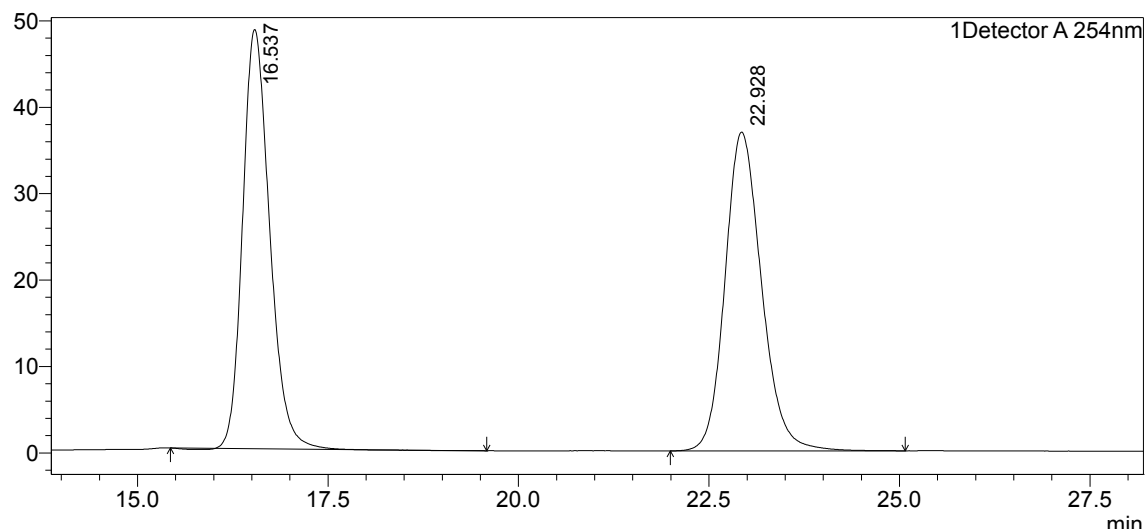

$<$ Peak Table>

\begin{tabular}{|c|c|c|c|c|c|c|}
\hline Peak\# & Ret. Time & Area & Height & Mark & Name & Area\% \\
\hline 1 & 16.537 & 1198694 & 48529 & $M$ & & 49.984 \\
\hline 2 & 22.928 & 1199469 & 36694 & $\mathrm{M}$ & & 50.016 \\
\hline Total & & 2398163 & 85223 & & & 100.000 \\
\hline
\end{tabular}

